\title{
THE DAMAGING EFFECTS OF NOISE AND ETHYL BENZENE ON HEARING
}

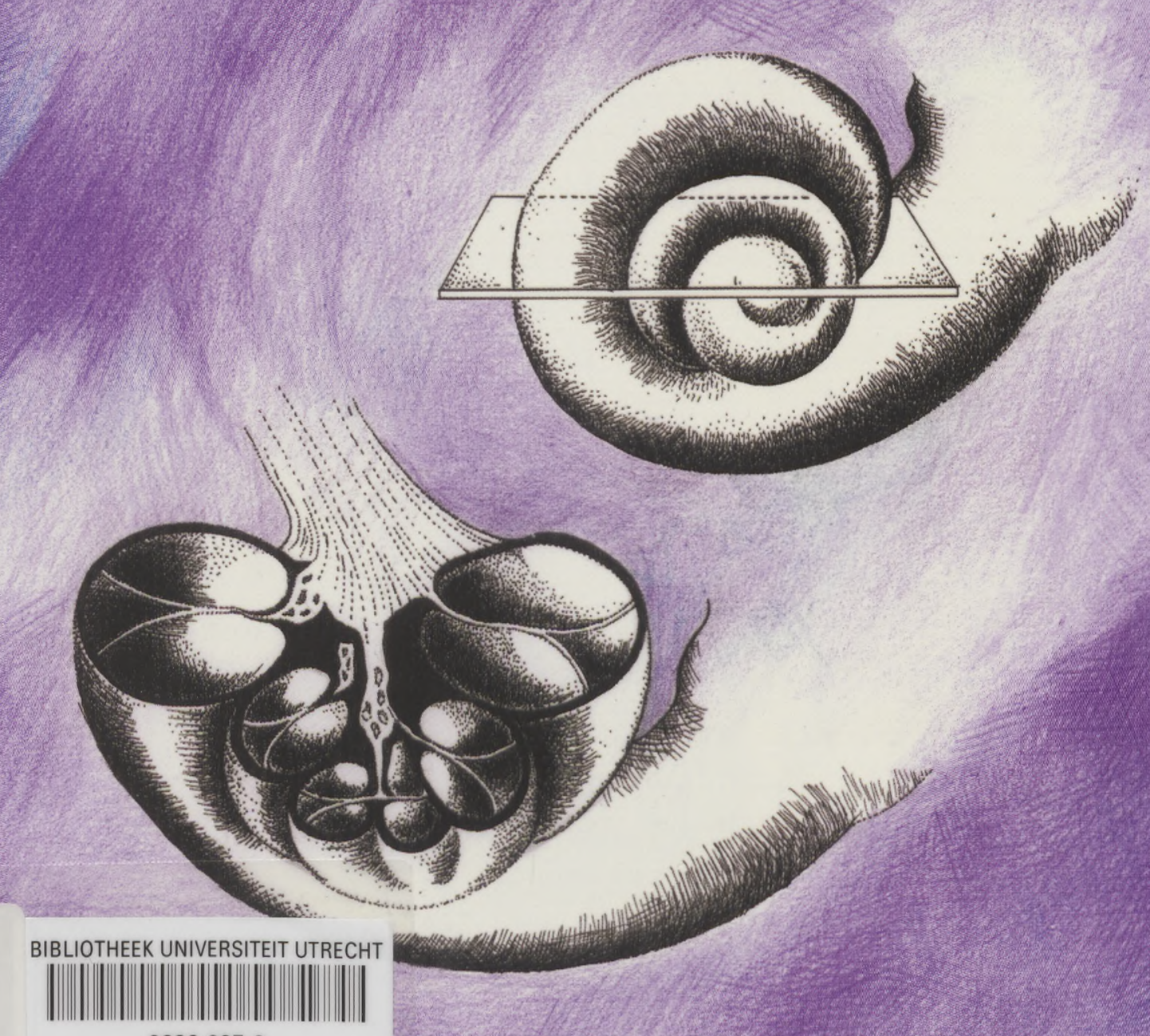

28389256

Natalie Cappaert 



\section{THE DAMAGING EFFECTS OF NOISE} AND ETHYL BENZENE ON HEARING

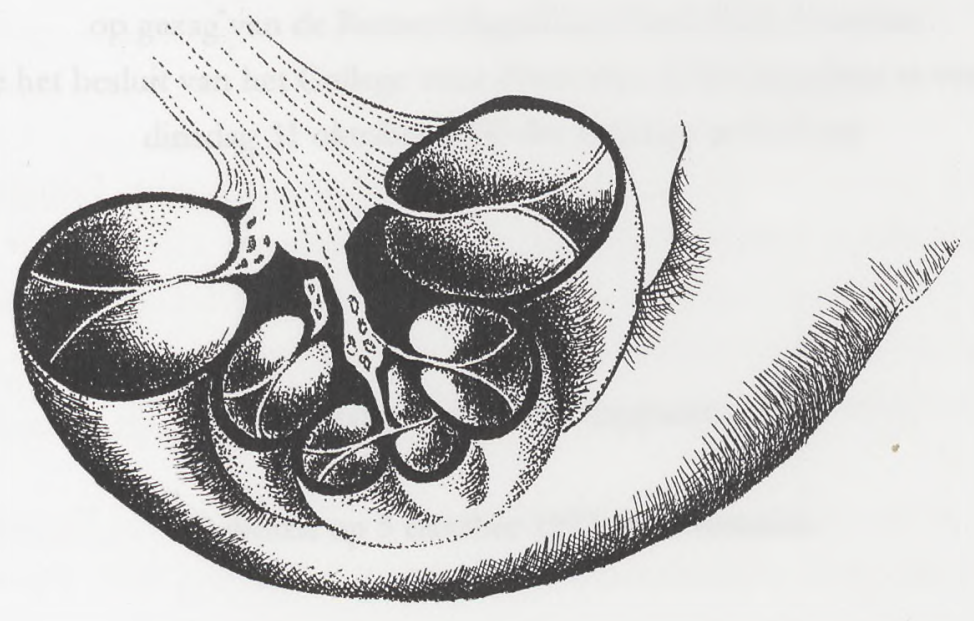




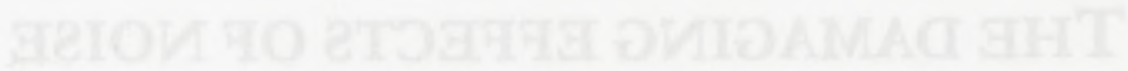

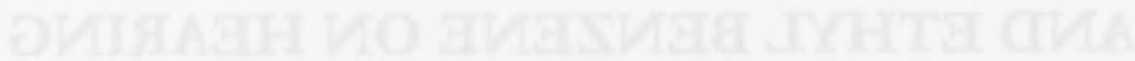




\section{THE DAMAGING EFFECTS OF NOISE}

\section{AND ETHYL BENZENE ON HEARING}

De schadelijke effecten van lawaai en ethylbenzeen op het gehoor. (met een samenvatting in het Nederlands)

Universiteits-

bibliotheek

UTRECHT

\section{PROEFSCHRIFT}

ter verkrijging van de graad van doctor aan de Universiteit Utrecht, op gezag van de Rector Magnificus, Prof. H.O. Voorma, ingevolge het besluit van het College voor Promoties in het openbaar te verdedigen op dinsdag 31 oktober 2000 des middags te 4.15 uur

door

Natalie Lijda Maria Cappaert

Geboren op 5 oktober 1973, te Terneuzen 


\section{INTRODUCTION}


Many people in industrialised countries are exposed to noise, especially at the workplace. This noise exposure can lead to noise-induced hearing loss (NIHL) which, thus, has become a significant health problem (Alberti, 1998). In the Netherlands, hearing loss ranks as one of the top ten work-related injuries (Passchier-Vermeer and Smoorenburg, 1994).

In addition, chemical agents present in the work environment may produce hearing loss. One class of these agents consists of the volatile organic solvents and includes compounds like toluene, styrene, trichloroethylene and ethyl benzene. Organic solvents are used as degreasers, adhesives, fuel-additives and thinners. Occupationally exposed humans primarily show effects like eye and throat irritation or dizziness. Long-term exposure may lead to permanent damage in the central nervous system with symptoms like memory and concentration deficits, and disturbances of emotional and psychomotor functions. Together these symptoms are known as the psycho-organic syndrome. In addition to the damage to the central nervous system, exposure to some solvents, especially the ones mentioned above, can lead to damage to the auditory system (ototoxic effects).

Exposure to noise and organic solvents can occur simultaneously in factories. Over the last decade a number of studies have investigated the effects of combined exposure to noise and organic solvents on hearing. Evidence is beginning to accumulate suggesting that a synergistic interaction may exist between noise and organic solvents. In this thesis, the central issue is whether or not there is such an interaction between noise and exposure to one of the most potent ototoxic compounds known, ethyl benzene. As a model to investigate this issue, we have used the peripheral auditory system of the rat and also the guinea pig.

\section{The peripheral auditory system}

The peripheral auditory system consists of three parts: the external ear, the middle ear and the inner ear. The first part, the external ear, includes the auricle and the ear canal. Sound waves in the air are caught by the auricle and propagated through the ear canal to the tympanic membrane. This membrane separates the outer ear from the middle ear. The sound sets the tympanic membrane in motion and the vibration is passed on to the three middle-ear ossicles (malleus, incus and stapes). The stapes is attached to the cochlea, which is a part of the inner ear. The ossicles compensate for 
the huge differences in acoustic impedance between air and water. They convert efficiently the air vibrations of the sound into fluid waves in the cochlea. The cochlea is responsible for the transduction of the sound-induced fluid waves into electrochemical impulses in the auditory nerve.

The cochlea is divided into three fluid-filled tube-like compartments: the scala vestibuli, the scala tympani and the scala media, which are coiled like a spiral. Two compartments, the scala vestibuli and the scala tympani, are filled with perilymph. The third compartment, the scala media, contains endolymph. A cross-section of the cochlea is given in Fig.1.

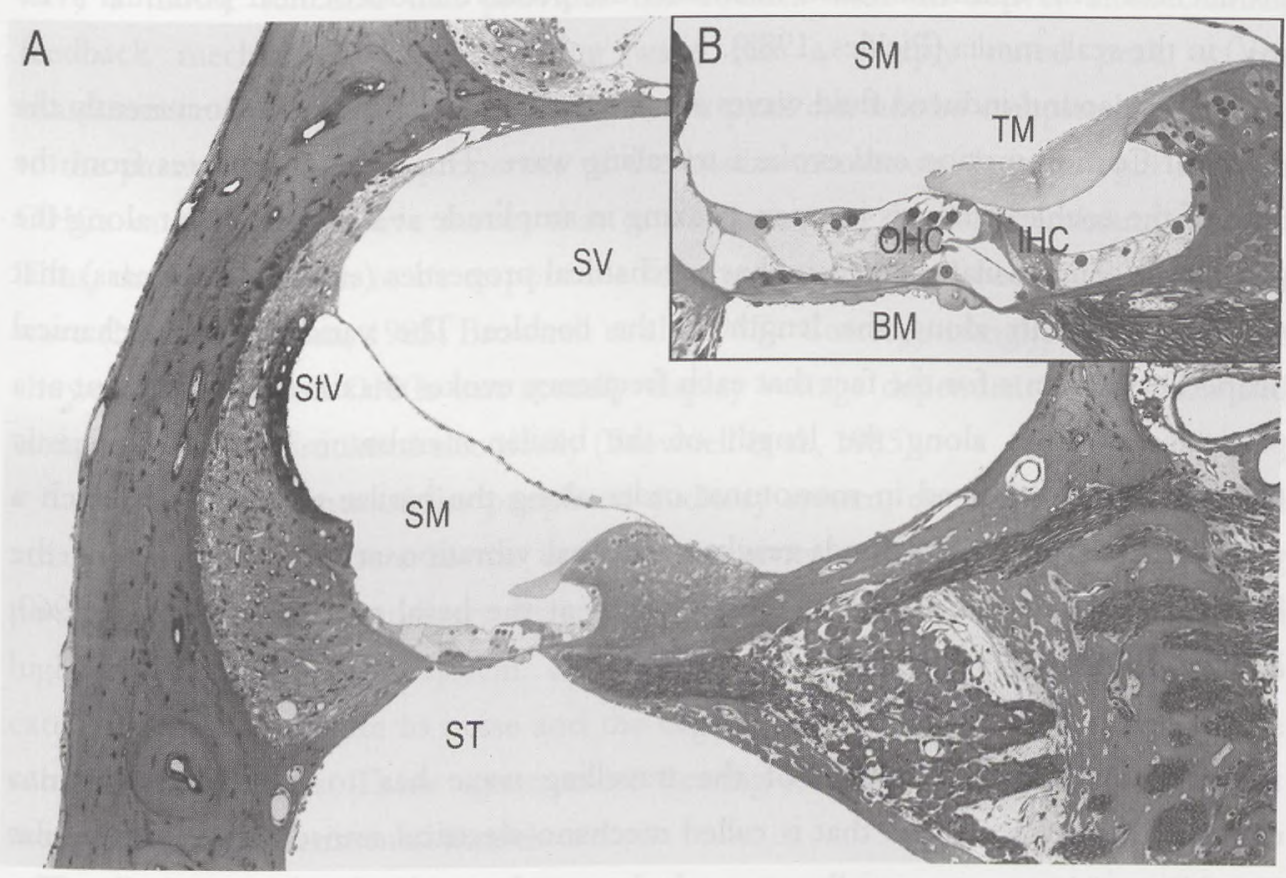

Fig. 1. A cross section of a turn of the cochlea (A) and the organ of Corti (B) in the rat. Panel $A$ and B are from different cochleas and turns. SM: Scala Media; SV: Scala Vestibuli; ST: Scala Tympani; StV: Stria Vascularis; TM: Tectorial Membrane; BM: Basilar Membrane; OHC: Outer Hair Cells; IHC: Inner Hair Cell.

The scala media is separated from the other two scalae by Reissner's membrane and the basilar membrane. The receptor organ of the inner ear, the organ of Corti, is positioned on the basilar membrane. Two types of receptor cells can be distinguished 
in the organ of Corti: the inner hair cells (IHCs) and outer hair cells (OHCs). The hair cells are arranged in three rows of OHCs and one row of IHCs. Both types of hair cells process a hair-like bundle of specialised stereocilia at the apical side of the cell, hence their name. The particular organisation in the organ of Corti brings about the essential division between the apical part of the hair cells, which bathes in endolymph, and the basolateral part, which bathes in perilymph. Perilymph is a plasma ultrafiltrate containing normal extracellular concentration of ions, i.e., high $\mathrm{Na}^{+}$and $\mathrm{Cl}^{-}$, low $\mathrm{K}^{+}$. The endolymph is a unique extracellular fluid, because of the high potassium concentration and the low sodium concentration. The stria vascularis in the lateral wall of scala media is a vascularised epithelium that is responsible for the endolymph production. This epithelium also maintains the positive endocochlear potential $(+80$ $\mathrm{mV}$ ) in the scala media (Pickles, 1988).

The sound-induced fluid waves set the basilar membrane and concurrently the organ of Corti in motion and evoke a travelling wave. This wave propagates from the base of the cochlea towards its apex, peaking in amplitude at a specific point along the membrane. The basilar membrane has mechanical properties (stiffness and mass) that vary systematically along the length of the cochlea. The variation in mechanical properties accounts for the fact that each frequency evokes maximal displacement at a particular position along the length of the basilar membrane. The characteristic frequencies are arranged in monotonic order along the basilar membrane in such a way that low-frequency sounds reach a maximal vibration at the apical part of the basilar membrane and high frequency sounds at the basal part (Von Bekesy, 1960; Patuzzi, 1996).

The mechanical energy of the travelling wave has to be transduced into electrical signals, a process that is called mechano-electrical transduction. The basilar membrane moves sequentially up and down when stimulated acoustically. The subsequent shearing motion between the organ of Corti and the tectorial membrane deflects the stereociliar bundles of the hair cells. The stereocilia have mechanically sensitive ion transduction channels that are sequentially opened and closed by the shearing motion. The general but somewhat simplified idea is that cations, predominantly potassium, flow into the hair cells when the basilar membrane moves towards the tectorial membrane. The influx is driven by the difference between the positive endocochlear potential in the endolymph and the negative potential inside the 
hair cell. Consequently, the cell becomes less negative and depolarises. Conversely, when the basilar membrane moves toward the scala tympani, the transduction channels close and subsequently the cell hyperpolarises (Hudspeth, 1989). Thus, the sequential alternations between pressure increase and decrease in an acoustical signal is transduced by the hair cells in a sequential alternation between depolarisations and hyperpolarisations.

These electrical signals are generated in both the IHCs and OHCs, but the neural information predominantly originates from the IHCs (Spoendlin, 1988), which receive at least $90-95 \%$ of the afferent auditory nerve innervation.

Nevertheless, the OHCs are crucial for the sensitivity. The OHCs appear to have a motor function that provides the cochlea with an active, biomechanical feedback mechanism. The travelling wave has a sharply tuned peak at the characteristic frequency. The amplitude of this peak can not be completely attributed to the passive mechanical properties of the basilar membrane. It is thought that the OHCs can generate active forces that can enhance the basilar membrane motion. Thus, the OHCs seem to be responsible for the sharply tuned peak in the travelling wave (Neely and Kim, 1983; Brownell et al., 1985). Correspondingly, in vitro studies showed that isolated OHCs can actually display voltage dependent contraction and elongation when stimulated electrically (Brownell et al., 1985).

All components of the peripheral auditory system, especially OHCs and the stria vascularis, contribute to the extreme sensitivity and high frequency resolving power of the peripheral auditory system. There is a drawback however, and that is the high vulnerability of this system. As already mentioned the vulnerability to two external agents, exposure to noise and the organic solvent ethyl benzene, will be the subject of this thesis. These agents cause hearing loss that is assessed by different methods in our experimental model.

\section{Assessment of hearing loss}

Auditory impairment induced by noise and organic solvents might be caused by lesions at different levels of the auditory system, e.g., middle ear, cochlea or central nervous system. The methods used in this study were chosen to assess the effects at different levels, but the emphasis will be on methods involving the inner ear. 


\section{Reflex modification audiometry}

Reflex modification audiometry (RMA) is a behavioural method for auditory assessment in animals. Brief low-intensity tone-stimuli (prepulses) will attenuate the magnitude of the startle response elicited by a subsequent high-intensity noise stimulus (Crofton et al., 1992). The prepulses are presented at different levels. The highest prepulse level that did not induce inhibition of the startle response was estimated from the measurements. This estimate was defined as the auditory threshold. RMA reflects the condition of the complete auditory pathway. So, effects detected with RMA might be due to peripheral damage but might just as well be connected with damage to auditory centres in the central nervous system.

\section{Electrocochleography}

When a tone burst is delivered to the ear, electric responses are evoked in the cochlea that can be recorded with a nearby electrode. These responses can be separated into several components. The cochlear microphonic (CM) originates with the receptor potential of the hair cells in response to sound-induced motion of the basilar membrane (forward transduction). Consequently, the CM reflects primarily the condition of the hair cells. However, it is important to be aware that damage to other structures, e.g., the stria vascularis that gives rise to the endocochlear potential, might also affect the CM.

The compound action potential (CAP) reflects the activity of afferent nerve fibres of the VIII'th nerve. The CAP is generated by a number of synchronously firing fibres at the onset of a stimulus (Pickles, 1988). Evaluating CAPs is a way to assess the function of the auditory periphery up to the VIII't nerve.

\section{Distortion product otoacoustic emissions}

The amplitude of a travelling wave depends on stimulus level in a highly nonlinear way. The amplitude of the wave grows with sound level at a much slower rate than would be expected from linearity (Dallos, 1992). Due to this nonlinearity, signals become distorted in the cochlea. When two tones $\left(f_{1}\right.$ and $\left.f_{2}\right)$ are applied simultaneously to the cochlea additional tones, not present in the stimulus, can be produced by this nonlinear transduction, resulting in intermodulation distortion. These additional tones or distortion products are emitted from the ear and are called 
distortion product otoacoustic emissions (DPOAEs). They can be measured with a sensitive microphone in the ear canal and are used for assessing cochlear sensitivity (Probst et al., 1991). The distortion product at the frequency of $2 \mathrm{f}_{1}-\mathrm{f}_{2}$ is the most prominent one (Probst et al., 1991; Patuzzi, 1996). These DPOAEs are probably generated by the active OHCs in a process that has been dubbed reverse transduction (Robles et al., 1991), to differentiate this OHC function from the forward transduction which gives rise to the CM.

\section{Histology}

Damage to the peripheral auditory system can also be assessed by examining the cochleas histologically. In this thesis, two quantitative analyses of the number of IHCs and OHCs present were performed. The first method, based on midmodiolar sections, determines the number of hair cell bodies present at a limited number of locations along the basilar membrane. The second method quantifies the number of hair cells present along the entire length of the basilar membrane (cytocochleograms) but is based only on the presence of stereociliar bundles. The number of hair cells missing is a measure of cochlear damage. The amount of hair cell loss and the functional impairment measured with the methods described above can be compared and correlated.

\section{Noise-induced hearing loss}

Exposure to noise may result in damage to the cochlea. The amount of hearing loss, the frequencies affected and reversibility of hearing loss depend on a complex interaction between frequency content, intensity, duration and temporal patterns of the sound (see: Borg et al., 1995; Salvi et al., 1995 for review). The reversible part of the threshold shift is defined as temporary threshold shift (TTS). In case of TTS, the auditory thresholds should return to pre-exposure values within a few hours to 20-30 days (Salvi et al., 1995). Any threshold shift left after this period is by definition called a permanent threshold shift (PTS).

Following a traumatic noise exposure, damage or loss of the hair cells is often seen. The OHCs are generally more vulnerable than the IHCs. Noise-induced changes can be found in the cell body as well as at the stereocilia. At least two mechanisms have been proposed for NIHL: mechanical injuries (Saunders et al., 1985) and damage 
due to metabolic exhaustion (Slepecky, 1986). It is believed that these two mechanisms both contribute to NIHL.

Mechanical injuries are characterised by severe disruption of the internal structures of the cell, followed by loss of cell homeostasis and lysis. Excessive stimuli may even cause movements that rupture the basilar membrane and the organ of Corti. This leads to a mixing of perilymph and endolymph. Stereocilia are also vulnerable and can appear floppy, disarrayed, fused, elongated or missing after excessive noise exposure. The stereocilia can lose their connection with the hair cell body by excessive shear motions.

Excessive sound stimulation can also effect the metabolism of the cell. Morphological changes in the endoplasmatic reticulum and mitochondria suggests defects in protein synthesis and energy production. When these changes are severe, the cell will deteriorate due to metabolic exhaustion resulting in distortion of cell homeostasis.

It is interesting to note that several authors have reported that the correlation between morphologically and physiologically detected noise-induced effects is not always straight forward (e.g., Borg et al., 1995).

\section{Organic solvents}

The peripheral auditory system has been demonstrated to be vulnerable to a number of chemical agents. Compounds used as drugs like aminoglycosides, cisplatin, loop diuretics and salicylates can affect hearing reversibly or irreversibly (Boettcher et al., 1987). Less well known is the fact that industrial chemicals like organic solvents can also impair the auditory system.

Some toluene-abusers showed signs of hearing loss or abnormal auditory brainstem responses (Fornazzari et al., 1983; Lazar et al., 1983). In addition, workers exposed to toluene vapours also exhibited signs of hearing impairment (Biscaldi et al., 1981; Morata et al., 1995).

An ototoxic effect of organic solvents in animals was first reported by Pryor et al. (1983). Rats exposed to toluene showed hearing loss in the mid-frequency region $(8-20 \mathrm{kHz}$ in this animal). This was evaluated by behavioural audiometry (Pryor et al., 1983) and confirmed by electrophysiological measurements (auditory brainstem responses) by Rebert et al. (1983). In rats Johnson and Canlon (1994a) found a 
depressing effect of toluene on DPOAEs in the mid-frequency range, suggesting a peripheral site of action. Morphological examination of the cochleas confirmed this suggestion. $\mathrm{OHC}$ loss was found in the mid-frequency region after toluene exposure. The largest loss of OHCs was found in the third or outer row. The second row was less damaged than the third row of OHCs, but more than the first row (Pryor, et al., 1984; Sullivan et al., 1989; Johnson and Canlon, 1994b; Campo et al., 1997; Lataye et al., 1999).

Similar observations of impaired hearing and $\mathrm{OHC}$ loss were reported for styrene. Rats exposed to styrene showed marked hearing loss assessed with behavioural and electrophysiological methods. The hearing loss was related to both exposure level and stimulus frequency (Pryor et al., 1987; Crofton et al., 1994). Like toluene, styrene has a peripheral site of action. Yano et al. (1992) found OHC loss in the basal and middle turns of the rat cochlea after exposure to styrene. Mäkitie (1997) and Lataye et al. (2000) confirmed these results. Furthermore, the pattern of OHC loss was the same as reported for toluene. The most severe loss was found in the third row of OHCs. The second and first row OHCs were less affected (Mäkitie, 1997; Loquet et al., 1999; Lataye et al., 2000).

In addition to toluene and styrene, trichloroethylene caused auditory impairment in rats (Rebert et al., 1991; Crofton and Zhoa, 1993; Jaspers et al., 1993; Rebert et al., 1993; Crofton et al., 1994; Fechter et al., 1998). Like other solvents, the functional loss is situated in the mid-frequency region. Trichloroethylene however, affected primarily the spiral ganglion cells, not the OHCs (Fechter et al., 1998).

The studies discussed above, concerning organic solvents and hearing, were performed on rats. Surprisingly, there is a remarkable difference in susceptibility between rats and guinea pigs. Guinea pigs did not show any or only very mild hearing loss after solvent exposure (Yamamura et al., 1983; Pryor and Rebert, 1993; Fechter, 1993; Campo et al., 1993). Other rodent species as mice and chinchilla showed mild effects of solvent exposure ( $\mathrm{Li}$ et al., 1992; Davis et al., 1996).

\section{Ototoxicity of ethyl benzene}

The solvent investigated in this thesis is ethyl benzene $\left(\mathrm{C}_{8} \mathrm{H}_{10}\right)$. The annual production of ethyl benzene amounts to 6.8 million metric tons in the U.S, which is twice as much as the annual production of toluene (American Chemical Society, 
1996). Ethyl benzene is primarily produced as an intermediate for styrene. However, a large amount is also consumed in connection with the use of xylene, as around 15$20 \%$ of technical xylene is ethyl benzene (Fishbein, 1985). The threshold limit value (TLV) is $100 \mathrm{ppm}$. Occupational exposure to ethyl benzene may occur through contact with gasoline, glues, varnishes, paints, synthetic rubbers and tobacco smoke.

Despite the high production figures of ethyl benzene, its ototoxicity is not extensively examined. To the best of our knowledge, Pryor and Rebert (1993) wrote the only report on the ototoxic effects of ethyl benzene. They showed that ethyl benzene was one of the most potent ototoxic organic solvents tested in their study comparing various solvents. They exposed rats to different concentrations of ethyl benzene. Hearing loss was assessed by auditory brainstem responses. The two higher concentrations affected hearing at 4,8 and $16 \mathrm{kHz}$. The just-significant ototoxic concentration was assessed at $250 \mathrm{ppm}$. This value is rather low when compared to figures for other solvents, e.g. $1601 \mathrm{ppm}$ for toluene and $439 \mathrm{ppm}$ for styrene (Pryor and Rebert, 1993).

\section{Combined exposure of organic solvents and noise}

One of the characteristics of ototoxic chemicals is that they may interact when administered together with noise or other ototoxic chemicals. The effects of combined exposure to noise and ototoxic chemicals are not necessarily predictable on the basis of their individual effects. Interactions between agents can be synergistic, additive, less than additive, equal to either agent alone or protective. In this thesis, the difference between additivity and synergism is important. In most of the reports in literature, additivity is described as the arithmetic sum of the effects observed by each individual exposure. This implies that the effect has to be quantified, e.g., expressed in $\mathrm{dB}$ hearing loss or percent hair cell loss. When the combined effect exceeds the arithmetic sum, the interaction is defined as synergistic.

Barregård and Axelsson (1984) suggested an interaction between noise and organic solvents in industrially exposed workers. They reported a more pronounced sensorineural hearing loss than the loss that would be predicted from exposure to noise alone and suggested that there may be an interaction between noise and solvents which enhances the hearing loss. Morata et al. (1991) explored the effects of simultaneous exposure to toluene and noise on hearing in occupationally exposed 
workers. The results of pure-tone audiometry indicated that the amount of hearing loss observed in the group exposed to both agents was significantly larger than the loss observed in the groups exposed to noise alone or toluene alone (Morata et al., 1991). However, Jacobsen et al. (1993) and Sass-Kortsak et al. (1995) found no evidence for interaction in workers exposed to noise and organic solvents.

Animal experiments have been carried out to study the combined effects of noise and organic solvents. The first animal studies were set up to study the effects of exposure to noise and toluene in rats, using auditory-evoked response audiometry (Johnson et al., 1988 and 1990). Rats were first exposed to toluene, followed by noise. Compared to the noise alone and toluene alone condition, the combination of noise and toluene resulted in a more than additive hearing loss in dB (Johnson et al., 1988). However, when the sequence of exposures was reversed, the hearing loss did not exceed the summated loss caused by noise alone or toluene alone (Johnson et al., 1990). In a more recent study (Lataye and Campo, 1997), the auditory deficits after simultaneous exposure to toluene and noise were tested with auditory brainstem responses in rats. This study confirmed the interaction between noise and toluene found by Johnson et al. (1988). The auditory deficits induced by combined exposure exceeded the summated loss (in $\mathrm{dB}$ ) of both agents applied alone. Moreover, the loss of OHCs after combined exposure to toluene and noise was enhanced as well (Lataye and Campo, 1997).

Furthermore, rats simultaneously exposed to trichloroethylene and noise showed a significantly larger threshold shift, measured with reflex modification audiometry, at $4 \mathrm{kHz}$ than the threshold shifts induced after noise alone and trichloroethylene alone. However, other frequencies tested $(8,16$ and $20 \mathrm{kHz})$ did not show threshold shifts larger than the arithmetic sum in $\mathrm{dB}$ due to combined exposure (Muijser et al., 2000). Mäkitie (1997) and Lataye et al. (2000) examined the effects of combined exposure to styrene and noise on hearing in the rat. The combination induced synergistic loss, expressed in $\mathrm{dB}$, of brainstem auditory evoked potentials. Furthermore, the percentage of $\mathrm{OHC}$ loss exceeded the summated loss due to exposure to one of the agents alone (Lataye et al., 2000). 


\section{Objectives of this study}

To the best of our knowledge the summary given above presents all the rat studies on combined exposure to noise and organic solvents. A common attribute of almost all studies is that they used high concentrations of organic solvents compared with government-permitted concentrations. Further, a study into the combined effect of the potentially ototoxic organic solvent ethyl benzene with noise is not among them. Because of the high potency of ethyl benzene, which provides the opportunity to study interactions under (near) realistic conditions, the study described in this thesis was performed.

In Chapter 2 the first investigation after the preliminary report of Pryor and Rebert (1993) into the ototoxicity of ethyl benzene in the rat is presented. The effects on hearing in rats of exposure to $800 \mathrm{ppm}$ ethyl benzene ( 8 hours/day for 5 consecutive days) were investigated, using reflex modification audiometry, CAP and $\mathrm{CM}$ measurements and hair cell counts.

Chapters 3 and 4 provide the dose-effect curves for ethyl benzene exposure (300, 400 and $550 \mathrm{ppm})$ and noise exposure (90, 100 and $110 \mathrm{~dB}$ SPL), respectively. One of the main purposes of these studies was to determine the minimum dose at which ethyl benzene exposure and noise exposure separately start to become ototraumatic.

In Chapter 5, the possible interaction between noise and ethyl benzene on hearing was investigated. Rats were exposed simultaneously to noise and ethyl benzene. The effects on the auditory system were determined using DPOAEs, CAPs and hair cell counts. We chose to apply two levels of noise (95 and $105 \mathrm{~dB}$ SPL) and two concentrations of ethyl benzene (300 and $400 \mathrm{ppm}$ ). The lowest level and concentration ( $95 \mathrm{~dB}$ SPL and $300 \mathrm{ppm}$ ethyl benzene, respectively) were close to the no-effect regimen. The higher level and concentration (105 dB SPL and $400 \mathrm{ppm}$ ethyl benzene, respectively) had a small effect on the CAPs. We focussed on small effects, because for occupational hygiene it is important to know whether or not two individual exposures, each considered to be safe, can be hazardous in combination.

In Chapter 6 differences in susceptibility between two animal models, rat and guinea pig, concerning ethyl benzene ototoxicity are discussed. The effects on hearing were determined by CAP measurements and the concentration of ethyl benzene in blood was determined after 1 and 3 days of exposure to ethyl benzene. 
In Chapters 7 and 8 , the results of investigations into the relation between the different measurements used in this thesis are discussed. In Chapter 7 we discuss the relation between the hearing loss derived from CAP measurements and OHC counts after exposure to noise and/or ethyl benzene in rats. Chapter 8 is an addendum and is the result of a joint study into the relation between electrophysiological measurements (CAPs) and behaviourally obtained reflex modification audiometry data. The animal data in this study were taken from in Chapter 2. 


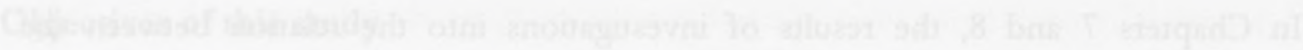

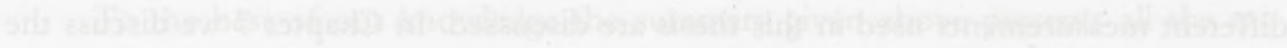

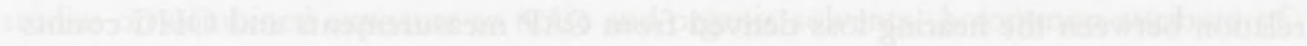

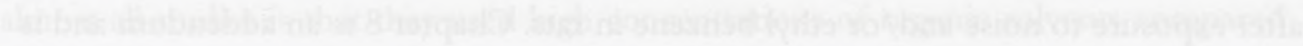

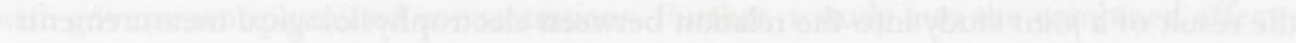

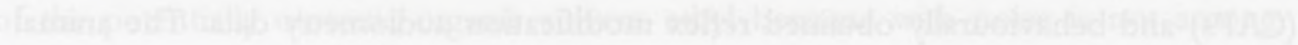

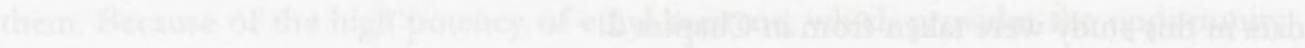

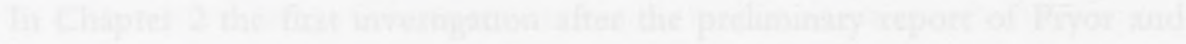




\section{THE OTOTOXIC EFFECTS OF ETHYL BENZENE IN RATS}

Exposure to organic solvents has been shown to be ototoxic in animals and there is evidence that these solvents can induce hearing loss in humans. In this study, the effects of inhalation of the possibly ototoxic solvent ethyl benzene on cochlear function and morphology were evaluated using three complementary techniques: 1) reflex modification audiometry (RMA), 2) electrocochleography and 3) histological examination of the cochleas.

Rats were exposed to either ethyl benzene ( $800 \mathrm{ppm}, 8$ hours/day for 5 days) or to control conditions. RMA threshold increased significantly by about $25 \mathrm{~dB}$ one and four weeks after the exposure, irrespective of the stimulus frequency tested $(4-24 \mathrm{kHz})$. Electrocochleography was performed between 8 and 11 weeks after exposure to the organic solvent. The threshold for the compound action potential increased significantly by 10 to $30 \mathrm{~dB}$ at all frequencies tested $(1-24 \mathrm{kHz})$. Histological examination of the cochlea showed outer hair cell loss, especially in the upper basal and lower middle turns (corresponding to the mid-frequency region) to an extent of $65 \%$. We conclude that exposure to $800 \mathrm{ppm}$ ethyl benzene for 8 hours/day during 5 days induces hearing loss in rats due to outer hair cell loss.

(Published as: Cappaert, Klis, Muijser, de Groot, Kulig, Smoorenburg, Hearing Research 1999; 137, 91-102) 


\section{INTRODUCTION}

Organic solvents are found in paints, varnishes, thinners, plastics and degreasers and are widely used in industries. Chronic exposure to some of these solvents can cause severe damage to the nervous system (Fornazzari et al., 1983; Spencer et al., 1985) and there is increasing evidence that exposure to some organic solvents is ototoxic in both humans and in animals (see: Rybak, 1992 and Pryor, 1994, for reviews).

Solvent abusers, exposed to high concentrations of toluene, showed signs of hearing loss, both in audiograms and in auditory brainstem responses (Metrick and Brenner, 1982; Lazar et al., 1983). Biscaldi et al. (1981) studied female employees in an electromechanical factory after acute exposure to high concentrations of toluene vapors. Three of the six workers showed hearing loss in the $3-6 \mathrm{kHz}$ region. Epidemiological research supported the idea that occupational exposure to organic solvents has an ototoxic effect on workers. In a Polish study, 40 trichloroethylene exposed workers, with urine levels of more than $40 \mathrm{mg}$ trichloroethylene per litre and exposure times of 1 to 23 years, were examined for auditory disorders. Hearing loss was detected in 26 of the examined workers (Szulc-Kuberska et al., 1976). In another study hearing was tested in workers who were exposed for about six years to a mixture of solvents, in which toluene was the major component (10-70 ppm). The adjusted relative risk for hearing loss in the exposed group was estimated to be 5 times greater, compared to the control group (Morata et al., 1993). Within a group of workers exposed to styrene for less than 1 month to 24 years (Muijser et al., 1988), the directly exposed workers (mean exposure: $138 \mathrm{mg} / \mathrm{m} 3$ ) had significantly more hearing loss than the indirectly exposed workers (mean exposure: $61 \mathrm{mg} / \mathrm{m} 3$ ). Sass-Kortsak et al. (1995), however, found no consistent relationship between styrene exposure and hearing loss. This lack of relationship might be due to confounding effects like age and noise exposure.

An ototoxic effect of organic solvents in laboratory animals was first reported by Pryor and co-workers (1983). Rats were exposed daily for 5 weeks, 14 hours/day, to 1200 or $1400 \mathrm{ppm}$ toluene by inhalation. Using a behavioural technique, hearing deficits were found predominantly in the mid-frequency range $(8-20 \mathrm{kHz})$. With the same exposure regimen, toluene-induced hearing loss was found also in auditory brainstem responses, suggesting a peripheral site of action (Rebert et al., 1983). This 
suggestion was confirmed by Johnson and Canlon (1994a). These authors applied the same compound (toluene applied at $1400 \mathrm{ppm}, 16$ hours/day for 8 days) to rats and found a depressing effect on the amplitude of the distortion product otoacoustic emission in the mid-frequency range, implying a loss in activity of the outer hair cells (OHCs). Histological examination confirmed this hypothesis. OHC loss was found in the mid-frequency region of the cochleas of rats exposed to toluene (Johnson et al., 1994b and Pryor et al, 1984; Sullivan et al., 1989; Campo et al., 1997). Similar observations of impaired hearing were reported after styrene exposure in rats. Inhalation of air containing styrene $(800,1000$ or $1200 \mathrm{ppm}, 14$ hours/day for 3 weeks) caused a marked hearing loss, which was related to both the exposure levels of styrene and stimulus frequency (Pryor et al., 1987). The higher frequencies were most affected, but measurements above $20 \mathrm{kHz}$ were not performed. A shorter exposure period, but higher exposure level, also produced hearing loss in rats. Exposure to 1600 ppm styrene ( 8 hours/day for 5 days) caused hearing loss at 8 and $16 \mathrm{kHz}$, but not at the lower and higher frequencies (Crofton et al., 1994). OHC loss was found by Yano and colleagues (1992) in the basal and middle turns of rats after exposure to $800 \mathrm{ppm}$ styrene (14 hours/day, 5 days/week for 3 weeks). Lower vapour levels of $600 \mathrm{ppm}$ styrene (Mäkitie, 1997) also resulted in OHC loss. Another organic solvent, trichloroethylene, applied at concentrations between 3000 and $4000 \mathrm{ppm}$, also produced a threshold elevation in the mid-frequency range measured behaviourally (Crofton and Zhao, 1993; Jaspers et al., 1993; Crofton et al., 1994; Fechter et al., 1998) and physiologically (Rebert et al., 1991; Rebert et al., 1993; Fechter et al., 1998). In contrast to toluene and styrene, trichloroethylene did not seem to affect the OHCs, but it caused a pronounced loss of the spiral ganglion cells (Fechter et al., 1998). This dissimilarity could be produced by the molecular structure of the solvent. Toluene and styrene are classified as aromatic hydrocarbons and trichloroethylene is a chlorinated hydrocarbon. Pryor (1994 and 1995) tried to investigate this structural requirement for organic solvents to cause hearing loss. He hypothesized that for benzene derivatives, the benzene ring needed a linear side chain (from methyl to n-propyl) to be ototoxic. Benzene itself was without effect and the only ototoxic compound of mixed xylene was p-xylene. For straight chain solvents tested thus far, only carbon-disulfide and trichloroethylene induced hearing loss. Although the three compounds described above are clearly ototoxic, it has to be kept in mind that several organic solvents do not induce hearing loss at all (Pryor, 1994). 
A compound that, to our knowledge, has not yet been extensively evaluated for ototoxic effects is ethyl benzene. Ethyl benzene (an aromatic hydrocarbon, $\mathrm{C}_{8} \mathrm{H}_{10}$, Threshold Limit Value: $100 \mathrm{ppm}$ ) is employed as a solvent, as an intermediate in the production of styrene, and in the plastic, rubber and petrol industries. Moreover, industrial grade xylene contains approximately $20 \%$ ethyl benzene. The annual production of ethyl benzene amounts to about 6.2 million metric tons in the U.S. alone (American Chemical Society, 1996). Thus, a considerable number of people is exposed to ethyl benzene on a daily basis.

This study was designed to address two issues. First, we wanted to evaluate the ototoxic potency of ethyl benzene in rats. Besides the obvious occupational safety aspects, it is interesting to study ethyl benzene as a model compound for organic solvent-induced ototoxicity because pilot experiments have shown that it causes ototoxic effects at concentrations much lower than other compounds (Pryor and Rebert, 1993). Second, we were interested in the mechanism of ototoxic action. As mentioned before, toluene (Pryor et al., 1984; Sullivan et al., 1989; Johnson and Canlon, 1994b; Campo et al., 1997) and styrene (Yano et al., 1992; Mäkitie, 1997) affect the ears of rats at a peripheral level, involving OHC loss. However, it is not clear whether OHC loss is the only effect responsible for hearing loss. Trichloroethylene, for example, exerts its ototoxic effect at the spiral ganglion level (Fechter et al., 1998). Given the well known neurotoxic properties of organic solvents more centrally located effects might also play a role. To our knowledge, there is no information yet on the site of ototoxic action of ethyl benzene, whether it is central or peripheral and, if peripheral, whether it involves OHCs or ganglion cells. On account of the chemical classification of ethyl benzene we expect an OHC involvement. Therefore, we investigated the ototoxicity of ethyl benzene by combining behavioural, electrophysiological and histological techniques.

\section{MATERIALS AND METHODS}

\section{Animals}

Thirty-two male rats (Wistar-derived strain Wag/Rij/Cpb/Hsd, obtained from Charles River, Germany), with an average weight of $200 \mathrm{~g}$ at the beginning of the experiment, were used. They were kept in wire mesh cages (4 animals/cage). Food and 
water were available ad libitum. Lights were on from 7:00 a.m. to 7:00 p.m. Animals were at random assigned to either control or treatment group. The animals were weighed weekly. Possible differences in weight gain between the two groups were tested with t-tests. Care and use of the animals reported on in this paper were approved by the Animal Care and Use Committee of the Faculty of Medicine, Utrecht University, under number DEC-GNK-95011.

\section{Ethyl benzene exposure}

The rats were exposed by inhalation to either air or ethyl benzene $(800 \mathrm{ppm}$ ethyl benzene, 8 hours/day for 5 days). They were placed in wire mesh cages in a stainless steel exposure chamber (Hazleton tank) from 8:30 a.m. till 4:30 p.m. Ethyl benzene (Merck, Schuchardt, 99\% pure) was vaporised at an elevated temperature and the saturated gas was injected into the air supply system, which operated at $20 \mathrm{~m}^{3} / \mathrm{hr}$. The gas concentration in the chamber was continuously monitored by a Brüel \& Kjær dual gas monitor (Type 3426). The concentration of ethyl benzene was kept within $10 \%$ of the desired concentration.

\section{Reflex modification audiometry}

Reflex modification audiometry (RMA) estimates auditory thresholds by recording the reduction of a noise evoked startle response when it is preceded by a single-frequency sound stimulus, the prepulse. The technique to measure reflex modification is described elsewhere (Jaspers et al., 1993) and is only summarised here. The animals were placed in a test cage, mounted on a force transducer assembly equipped with strain gauges (Kyowa, Tokyo, Japan, Model KFC-5C1-11). After a 10 minute adaptation period the rat received a total of 800 trials. The test session was divided into 25 blocks of 32 trials. Each block included 26 trials with prepulses, of 2 frequencies and 13 intensities. In addition, 6 blank trials in which the prepulse was absent were also presented. The order of presentation of the trials within each block was such that blank trials and trials with a prepulse frequency were regularly alternated. The startle-eliciting stimulus, which is presented in each trial, was a noise burst of $40 \mathrm{~ms}$ at $110 \mathrm{~dB}$ SPL. It was preceded by a $40 \mathrm{~ms}$ prepulse presented $100 \mathrm{~ms}$ before the onset of the startle eliciting noise burst. The prepulse was a Hanning shaped sinusoidal tone burst of $4,8,12,16,20$ or $24 \mathrm{kHz}$, presented at 13 levels with 
$6 \mathrm{~dB}$ increments. The frequencies were tested in 3 pairs ( 4 and $16 \mathrm{kHz} ; 8$ and $20 \mathrm{kHz}$; 12 and $24 \mathrm{kHz}$ ). Testing of 32 animals with the 3 pairs of prepulse frequencies was performed in 6 mornings and 6 afternoons on 6 consecutive days. Half of the rats of one group was tested in the morning and the other half was tested in the afternoon. The rats were always tested at the same time of the day and the same sequence of frequencies was kept, to minimize the variations in startle response between testing days. No more than 1 frequency pair was tested at one day. The auditory thresholds for all tested frequencies were obtained using a previously described model (Crofton, 1992; Jaspers et al., 1993). In the model the highest prepulse level that did not induce inhibition of the startle response was estimated. This estimate was defined as the auditory threshold. Auditory thresholds were determined for all frequencies one week before exposure to ethyl benzene (pre-condition) and one and four weeks afterwards (post_1 and post_4 condition).

The threshold shift was determined as follows: the mean threshold of the post_1 and post_ 4 condition of the control group (averaged over all control animals) minus the mean threshold of the post_1 and post_4 condition per animal. These threshold shifts per exposed animal were averaged and used as the threshold shift per frequency.

The behavioural data were evaluated by means of analysis of variance (ANOVA). The effect of ethyl benzene was tested as a between-subjects factor and frequency and time (pre-condition, post_1 and post_4) were within-subject factors.

\section{Electrocochleography}

Electrocochleography was performed 8 to 11 weeks after the exposure. The method was described previously for guinea pigs (Stengs et al., 1997). Only minor modifications were necessary to accommodate rats in our set-up. The rat was anaesthetised with an intramuscular injection of Thalamonal $(0.1 \mathrm{ml} / 100 \mathrm{~g}$ body weight; Thalamonal contains $2.5 \mathrm{mg} / \mathrm{ml}$ droperidol and $0.05 \mathrm{mg} / \mathrm{ml}$ fentanyl). This was followed by ventilation with a gas mixture containing $33 \% \mathrm{O}_{2}, 66 \% \mathrm{~N}_{2} \mathrm{O}$ and $1 \%$ Halothane through a trachea cannula. During surgery and experimental procedures, heart rate was monitored and rectal temperature was kept at $38^{\circ} \mathrm{C}$. Both the right and left cochlea were exposed through a ventrolateral approach. Auditory evoked responses were recorded differentially with a silverball electrode at the apex of the 
cochlea. A reference electrode was placed in the muscles of the neck. Stimulus generation and data acquisition were controlled by computer. Trains of $8 \mathrm{~ms}$ tone bursts of $1,2,4,8,12,16$ and $24 \mathrm{kHz}$ were presented, with cosine shaped rise and fall time of $1 \mathrm{~ms}(2 \mathrm{~ms}$ at $1 \mathrm{kHz} ; 1.5 \mathrm{~ms}$ at $2 \mathrm{kHz}$ ). Consecutive tone bursts were presented with alternating polarity. The inter burst-interval was $99 \mathrm{~ms}$. The responses were amplified, band-pass filtered ( $-12 \mathrm{~dB} /$ octave, $-3 \mathrm{~dB}$ at $100 \mathrm{~Hz}$ and $10 \mathrm{kHz}), \mathrm{AD}$ converted at a sample frequency of $33.3 \mathrm{kHz}$ and averaged (max. 500x). The responses, averaged separately for each polarity, were saved for off-line analysis.

The compound action potential (CAP) was obtained by addition of the responses of opposite polarity, the cochlear microphonics $(\mathrm{CM})$ by subtraction. CAP amplitude was defined as the difference between the highest negative peak and the highest positive peak in the complex waveform. The threshold is defined as the isoresponse level at which an acceptable signal to noise ratio is reached. We set this criterion at $1 \mu \mathrm{V}$. The threshold shift is defined as the difference in auditory threshold (based on the CAP data) between the control and exposed group. The CM was determined as the peak-to-peak value of the sinusoidal response. CM was not measured at 12, 16 and $24 \mathrm{kHz}$ because these frequencies exceeded the upper cut-off frequency of our measurement system.

All CAP and CM data were logarithmically transformed before analysis to improve homogeneity of variance. Subsequently, the data from left and right ears together were averaged per stimulus condition. ANOVA was performed with ethyl benzene exposure as a between-subjects factor and frequency and level of stimulation as within-subject factors.

\section{Histology}

Immediately after electrocochleography, the cochleas were fixed by transcardial perfusion with a chilled tri-aldehyde fixative consisting of $3 \%$ glutaraldehyde, $2 \%$ formaldehyde, $1 \%$ acrolein, $2.5 \%$ dimethylsulfoxide in $0.1 \mathrm{M}$ sodium cacodylate buffer ( $\mathrm{pH}$ 7.4), followed by overnight immersion in the same fixative at $4^{\circ} \mathrm{C}$. The cochleas were further processed according to the routine method used for guinea pigs (De Groot et al., 1987). Semithin $(1 \mu \mathrm{m})$ midmodiolar sections were cut and stained with $1 \%$ methylene blue, $1 \%$ azur II in $1 \%$ sodium tetraborate. Sections were studied under the light microscope (Zeiss Axiophot photomicroscope). In a midmodiolar section of 
the rat cochlea, the organ of Corti was examined at five locations separated by a halfturn distance. Hair cell counts were performed at each of these five locations in both the left and the right cochlea, using 5-6 subsequent sections. If a hair cell is not seen in these 5-6 subsequent $1 \mu \mathrm{m}$ sections, it can be reliably assumed that the cell is not present. OHC loss was expressed as the percentage of remaining OHCs per cross sectioned half turn ( 2 transections each for the basal and middle turns; 1 transection for the apical turn, see Fig. 1), relative to the expected number of OHCs. All OHCs counts were performed by 2 investigators, independently of one another, in a singleblind fashion. An estimate was made of the characteristic frequencies of the locations along the basilair membrane at which the OHCs were counted (Fig. 1).

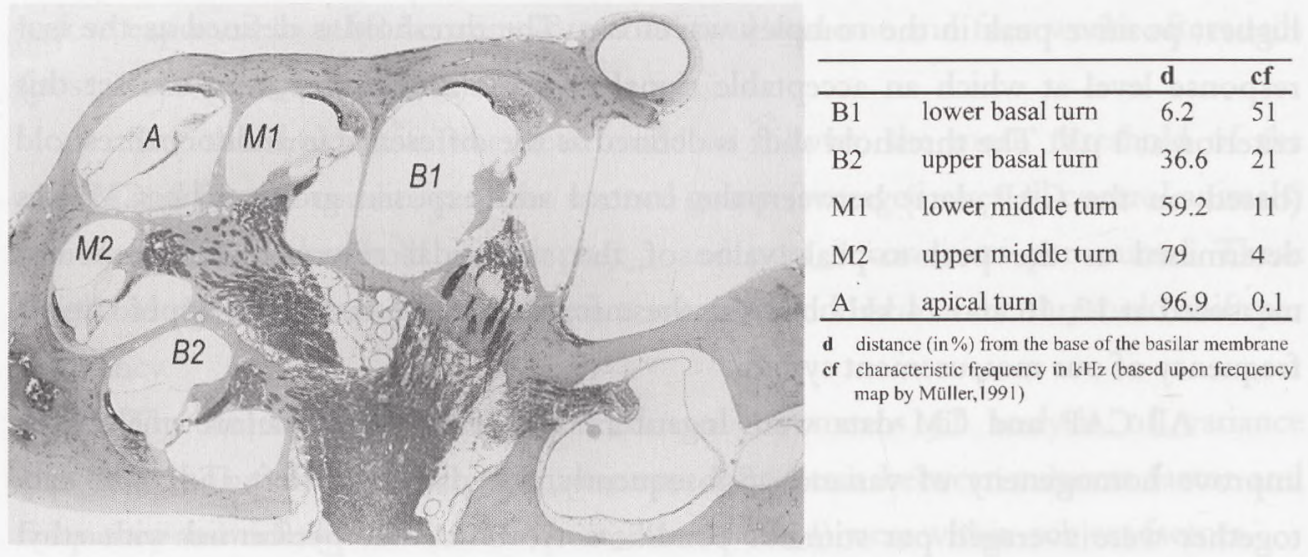

Figure 1. Midmodiolar section of the cochlea. Two transections each for basal (B1 and B2) and middle turns (M1 and M2), one transection for the apical turn (A). The distance (d) is determined (in \%) from the base of the basilar membrane and the characteristic frequency (cf) in $\mathrm{kHz}$ is computed (based upon frequency map by Müller, 1991).

The corresponding frequencies were calculated to be $52,21,11,4$ and $0.1 \mathrm{kHz}$, respectively, according to a frequency map published by Müller (1991). The average length of the basilar membrane in three cochleas of $2 \mathrm{Wag} / \mathrm{Rij}$ rats was estimated to be $12.9 \mathrm{~mm}$.

The results for the left and right cochleas were pooled and averaged per location. ANOVA was used for statistical evaluation of the OHC counts. Ethyl benzene exposure was a between-subjects factor, cochlear location a within-subjects 
factor. Significance of the differences in the OHC counts between the cochlear halfturns was tested post hoc using Tukey's HSD test.

\section{RESULTS}

\section{General health}

The rats exhibited no overt signs of ill health after ethyl benzene exposure. At the end of the exposure period the body weight of the ethyl benzene-treated animals did not differ from the control animals ( $\mathrm{t}$-test, $\mathrm{P}=0.11$ ). One animal in the control group fractured its nose by accident. After this accident it was not sufficiently fit for electrocochleography. However, its cochleas were collected and included in the histological examinations.

\section{Reflex modification audiometry}

Fig. 2 shows the auditory thresholds, measured with RMA. Fig 2A shows that there was no difference between the group of animals that was assigned to be the control group and the group that was going to be exposed to ethyl benzene. ANOVA confirmed that the thresholds in both groups were similar. Fig $2 \mathrm{~B}$ shows the thresholds in both these groups one week and four weeks post exposure to ethyl benzene. The thresholds of the exposed group shifted to higher levels at all frequencies. The average effect was $26 \mathrm{~dB}$. ANOVA, performed on the data from both groups with all frequencies and time conditions included, showed significant main effects of ethyl benzene, frequency and time. No interaction was found between ethyl benzene and frequency, thus ethyl benzene had a frequency-independent effect on the RMA thresholds (Table 1). However, a significant interaction between ethyl benzene and time was present (see Table 1). This interaction was further pursued with the Tukey HSD post hoc test including the factors ethyl benzene and time. This test showed significant differences $(\mathrm{P}<0.01)$ between the threshold measured after one and four weeks in the exposed group and the pre-exposure threshold in both the control and the exposed group. 


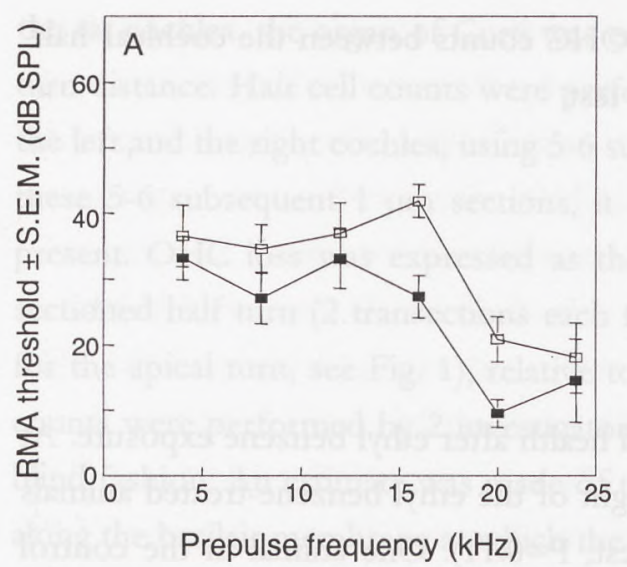

$\square \quad$ Control

- To be exposed

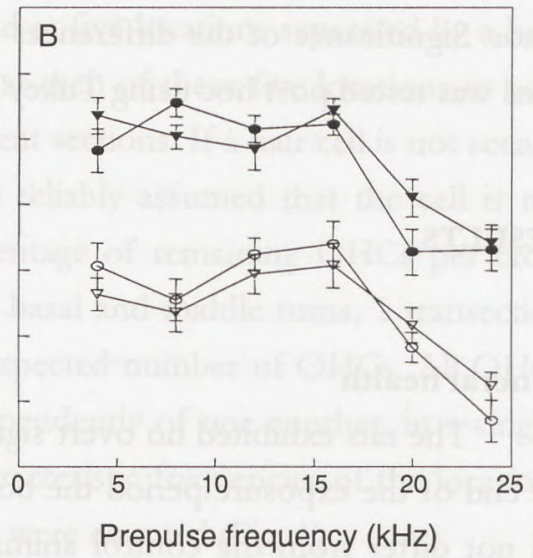

$\nabla$ Post_1 control

$\checkmark$ Post_1 ethyl benzene

- Post_4 control

- Post_4 ethyl benzene

Figure 2. Reflex modification audiometry (RMA) thresholds of rats measured before $(A)$ and after (B) exposure. RMA thresholds deteriorated in the group exposed to 800 ppm ethyl benzene ( 8 hours/day for 5 days). This deterioration was significant one week after exposure (post_1) and did not progress further nor did thresholds recover up to four weeks after exposure (post_4). In the control group the thresholds did not change over this period.

Table 1: Statistical evaluation (ANOVA) of the reflex modification audiometry data Ethyl benzene exposure is a between-groups factor and frequency and time are withingroup factors.

\begin{tabular}{lcc}
\hline & $\mathrm{F}$ & $\mathrm{P}$ \\
\hline ethyl benzene & $\mathrm{F}_{(1,29)}=14.3$ & $\mathrm{P}<0.01$ \\
frequency & $\mathrm{F}_{(5,145)}=2.5$ & $\mathrm{P}<0.05$ \\
time & $\mathrm{F}_{(2,58)}=6.7$ & $\mathrm{P}<0.01$ \\
ethyl benzene $\bullet$ frequency & n.s. & n.s. \\
ethyl benzene $\bullet$ time & $\mathrm{F}_{(2,58)}=12.3$ & $\mathrm{P}<0.01$ \\
frequency $\bullet$ time & n.s. & n.s. \\
ethyl benzene $\bullet$ frequency $\bullet$ time & n.s. & n.s. \\
\hline
\end{tabular}

Also, the differences between the post exposure thresholds of the exposed group and the post exposure thresholds of the control group were statistically significant $(\mathrm{P}<0.01)$. To investigate the effect of ethyl benzene in time, ANOVA was performed per group (the exposed and control group separately), with frequency and time as 
within-group factors. A significant effect of time was found in the exposed group only $\left(\mathrm{F}_{(2,30)}=64.0 ; \mathrm{P}<0.01\right)$. This effect was pursued further with the Tukey HSD post hoc test which showed differences between the pre and post_1 condition and the pre and post_4 condition (both $\mathrm{P}<0.01$ ). No differences in threshold were found between 1 week and four weeks after exposure.

\section{Electrocochleography}

CAP amplitude growth curves were measured 8 to 11 weeks after the exposure and are shown in Fig. 3. The most apparent effect of ethyl benzene was a decrease of the CAP amplitudes, at all frequencies tested. However, this decrease was extremely level-dependent and virtually absent, or even reversed $(4 \mathrm{kHz})$ at the highest stimulus levels tested. ANOVA, performed on the whole data set, averaged for left and right ears, as presented in Fig. 3, showed main effects of ethyl benzene, frequency and level of stimulation (Table 2). There was no significant interaction between ethyl benzene and frequency and thus, no frequency-dependent effect of ethyl benzene. A salient interaction was found between ethyl benzene and level of stimulation, which confirms the visually observed level dependence of the ethyl benzene effect. This interaction resembles the psychophysically defined phenomenon of "loudness recruitment" in patients with sensorineural hearing loss. Average threshold shift at $1 \mu \mathrm{V}$ was $20 \pm 2.2$ $\mathrm{dB}$ (mean \pm S.E.M.), at $10 \mu \mathrm{V}$ it was $8.1 \pm 2.1 \mathrm{~dB}$ (mean \pm S.E.M.); full recruitment (no difference between CAP amplitude of control and exposed group) was reached at $25 \pm 7.2 \mu \mathrm{V}$ (mean \pm S.E.M.).

Table 2: Statistical evaluation (ANOVA) of the compound action potential data. Ethyl benzene exposure is the between-groups factor and frequency and level of stimulation are within-group factors.

\begin{tabular}{lcc}
\hline & $\mathrm{F}$ & $\mathrm{P}$ \\
\hline ethyl benzene & $\mathrm{F}_{(1,29)}=71.2$ & $\mathrm{P}<0.01$ \\
frequency & $\mathrm{F}_{(6,174)}=163.8$ & $\mathrm{P}<0.01$ \\
level of stimulation & $\mathrm{F}_{(8,232)}=1587.6$ & $\mathrm{P}<0.01$ \\
ethyl benzene $\cdot$ frequency & n.s. & n.s. \\
ethyl benzene $\cdot$ level of stimulation & $\mathrm{F}_{(8,232)}=50.8$ & $\mathrm{P}<0.01$ \\
frequency $\bullet$ level of stimulation & $\mathrm{F}_{(48,1392)}=17.6$ & $\mathrm{P}<0.01$ \\
ethyl benzene $\cdot$ frequency $\cdot$ level of stimulation & $\mathrm{F}_{(48,1392)}=10.7$ & $\mathrm{P}<0.01$ \\
\hline
\end{tabular}



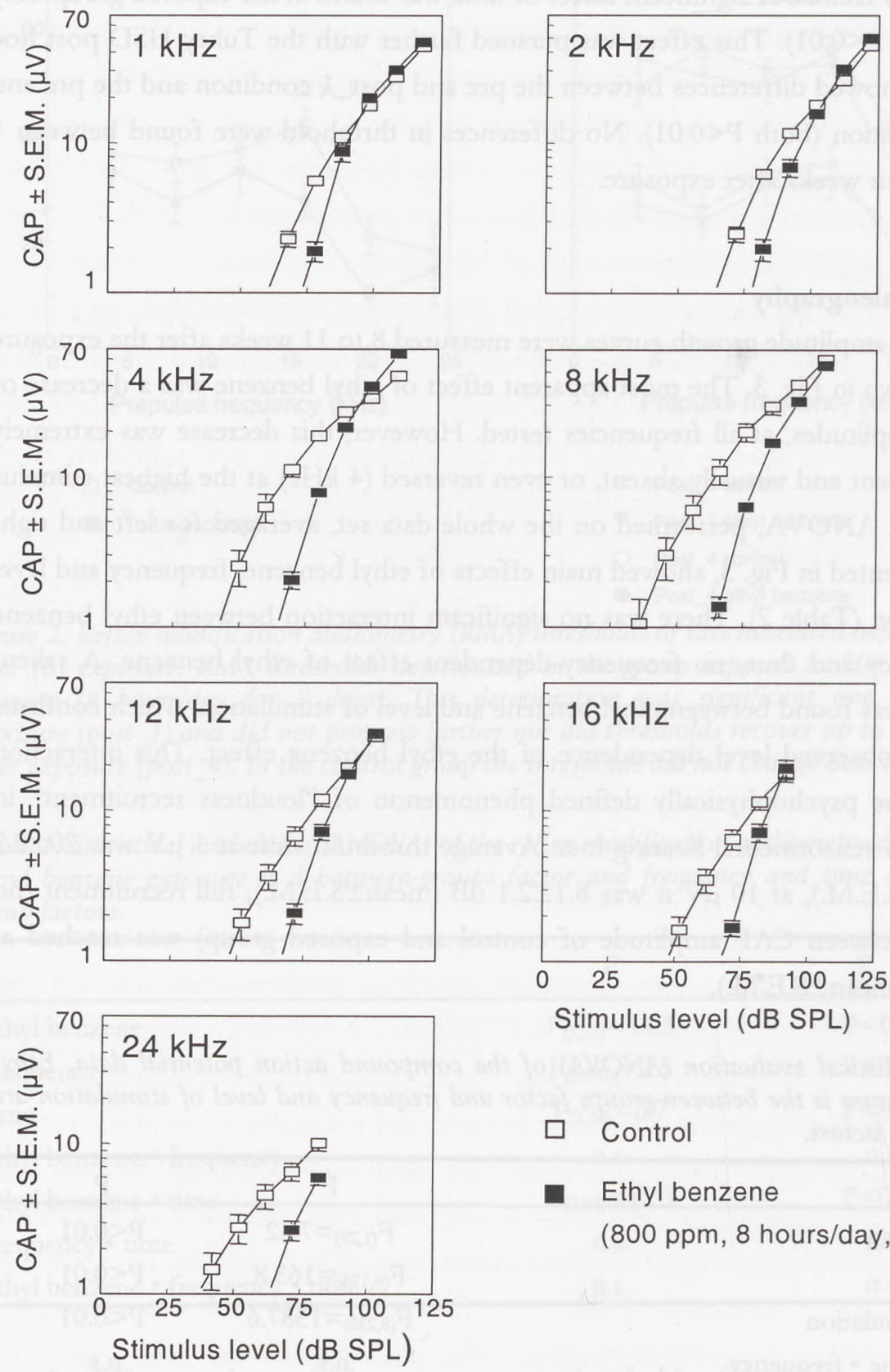

Stimulus level (dB SPL)

$\square$ Control

- Ethyl benzene (800 ppm, 8 hours/day, 5 days)

Figure 3. Effects of ethyl benzene on CAP amplitude growth curves at the indicated frequencies. Note the level dependence of the effect of ethyl benzene on the CAP amplitude. 
The effects of ethyl benzene on the CM amplitude are represented in the CM growth curves, arranged per frequency in Fig. 4. The most obvious effect of ethyl benzene is an apparently vertical shift of the CM growth curves. ANOVA, performed on the CM data, averaged for left and right ears, with all frequencies included, showed main effects of ethyl benzene, frequency and level of stimulation on CM amplitude (Table 3). There was no significant interaction between ethyl benzene and frequency. Thus, again no frequency-dependent effect of ethyl benzene on CM growth curves was found. A significant interaction between ethyl benzene and level of stimulation was found.

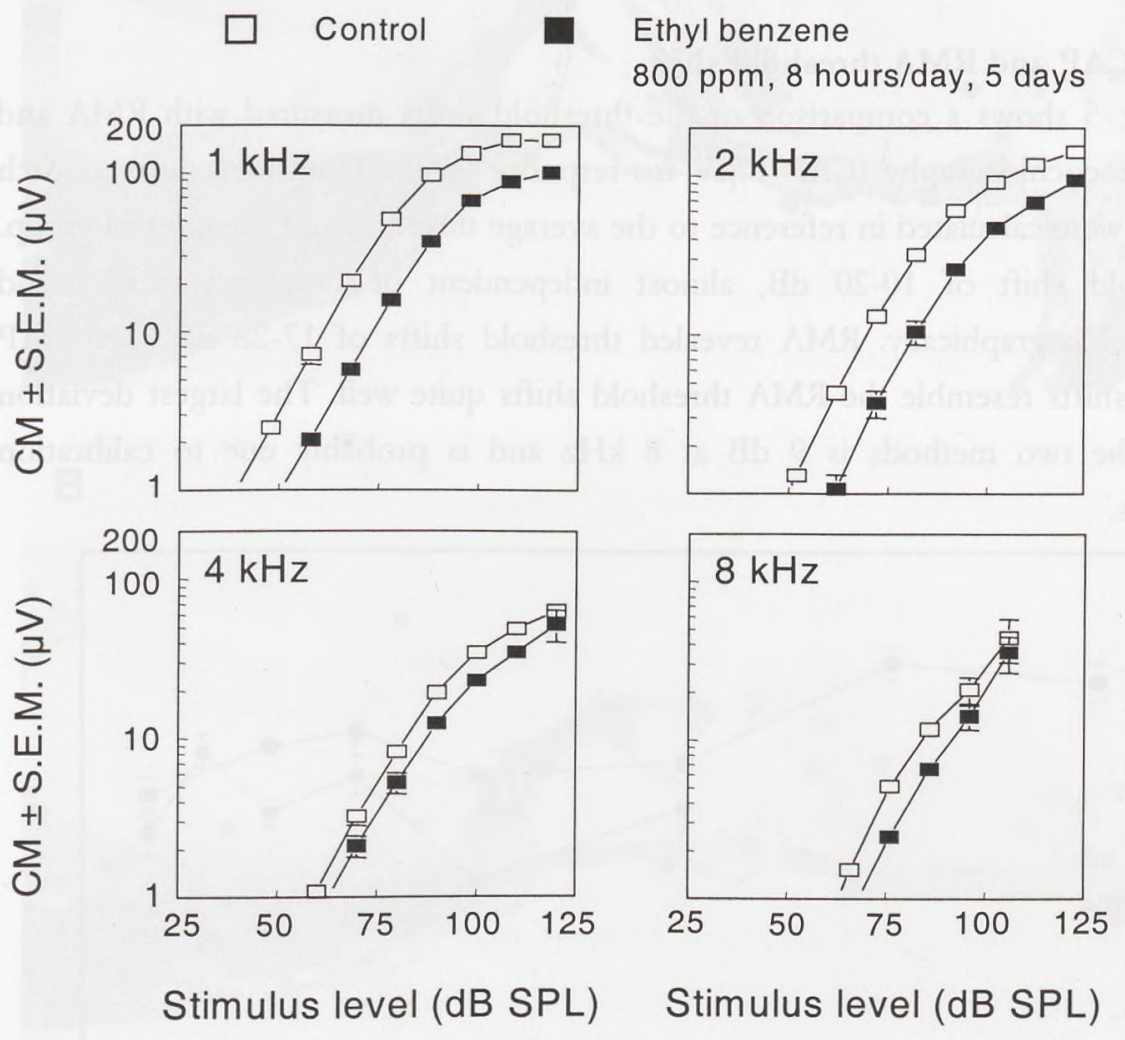

Figure 4. Effects of ethyl benzene on the CM amplitude growth curves at the indicated frequencies. 
Table 3: Statistical evaluation (ANOVA) of the cochlear microphonics data. Ethyl benzene exposure is the between-groups factor and frequency and level of stimulation are withingroup factors.

\begin{tabular}{lcc}
\hline & $\mathrm{F}$ & $\mathrm{P}$ \\
\hline ethyl benzene & $\mathrm{F}_{(1,29)}=34.6$ & $\mathrm{P}<0.01$ \\
frequency & $\mathrm{F}_{(3,87)}=425.7$ & $\mathrm{P}<0.01$ \\
level of stimulation & $\mathrm{F}_{(5,145}=1116.0$ & $\mathrm{P}<0.01$ \\
ethyl benzene $\cdot$ frequency & n.s. & n.s. \\
ethyl benzene $\cdot$ level of stimulation & $\mathrm{F}_{(5,145)}=10.8$ & $\mathrm{P}<0.01$ \\
frequency $\cdot$ level of stimulation & $\mathrm{F}_{(15,435)}=78.4$ & $\mathrm{P}<0.01$ \\
ethyl benzene $\cdot$ frequency $\bullet$ level of stimulation & n.s. & n.s. \\
\hline
\end{tabular}

\section{Relation CAP and RMA threshold shift}

Fig. 5 shows a comparison of the threshold shifts measured with RMA and with electrocochleography (CAP $1 \mu \mathrm{V}$ iso-response level). Threshold shifts at each frequency were calculated in reference to the average thresholds of the control group. A threshold shift of 10-20 dB, almost independent of frequency, was found electrochochleographically. RMA revealed threshold shifts of 17-28 dB. The CAP threshold shifts resemble the RMA threshold shifts quite well. The largest deviation between the two methods is $9 \mathrm{~dB}$ at $8 \mathrm{kHz}$ and is probably due to calibration differences.

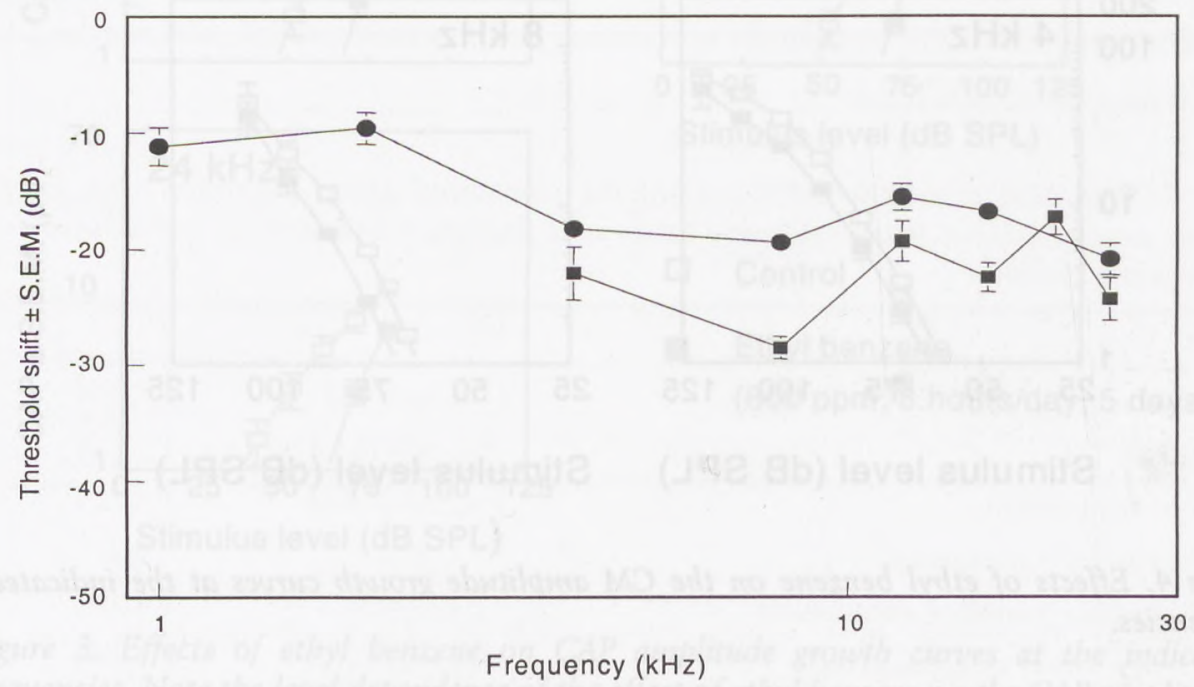

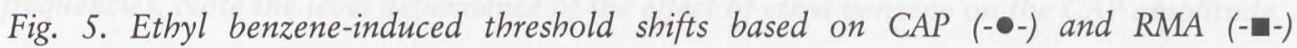
measurements as a function of frequency. 


\section{Histology}

Two representative light micrographs of the organ of Corti from the lower part of the middle turn of a control animal (A) and of an exposed animal (B) are shown in Fig. 6. All OHCs are present at this location in the cochlea of the control animal. In contrast, there is only one $\mathrm{OHC}$ remaining in the same area of the treated animal.
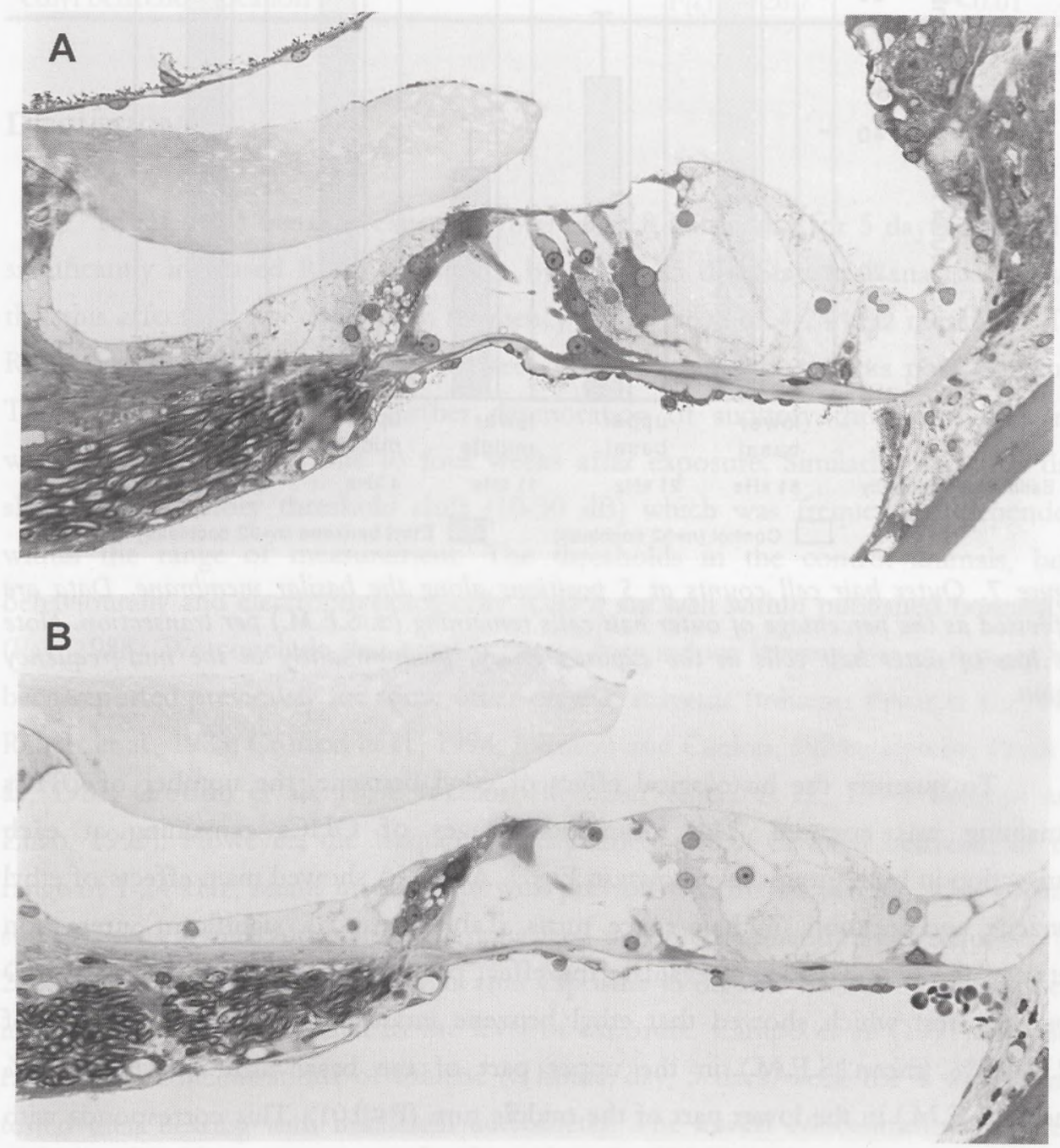

Figure 6. Light micrographs showing the organ of Corti as observed in the lower part of the middle turn of the rat cochlea. All outer hair cells in the control animal (A) were present. In the rat exposed to ethyl benzene (B) two of the three outer hair cells have disappeared and the remaining outer hair cell has a shrunken balloon-like appearance but still adheres to the reticular lamina. 


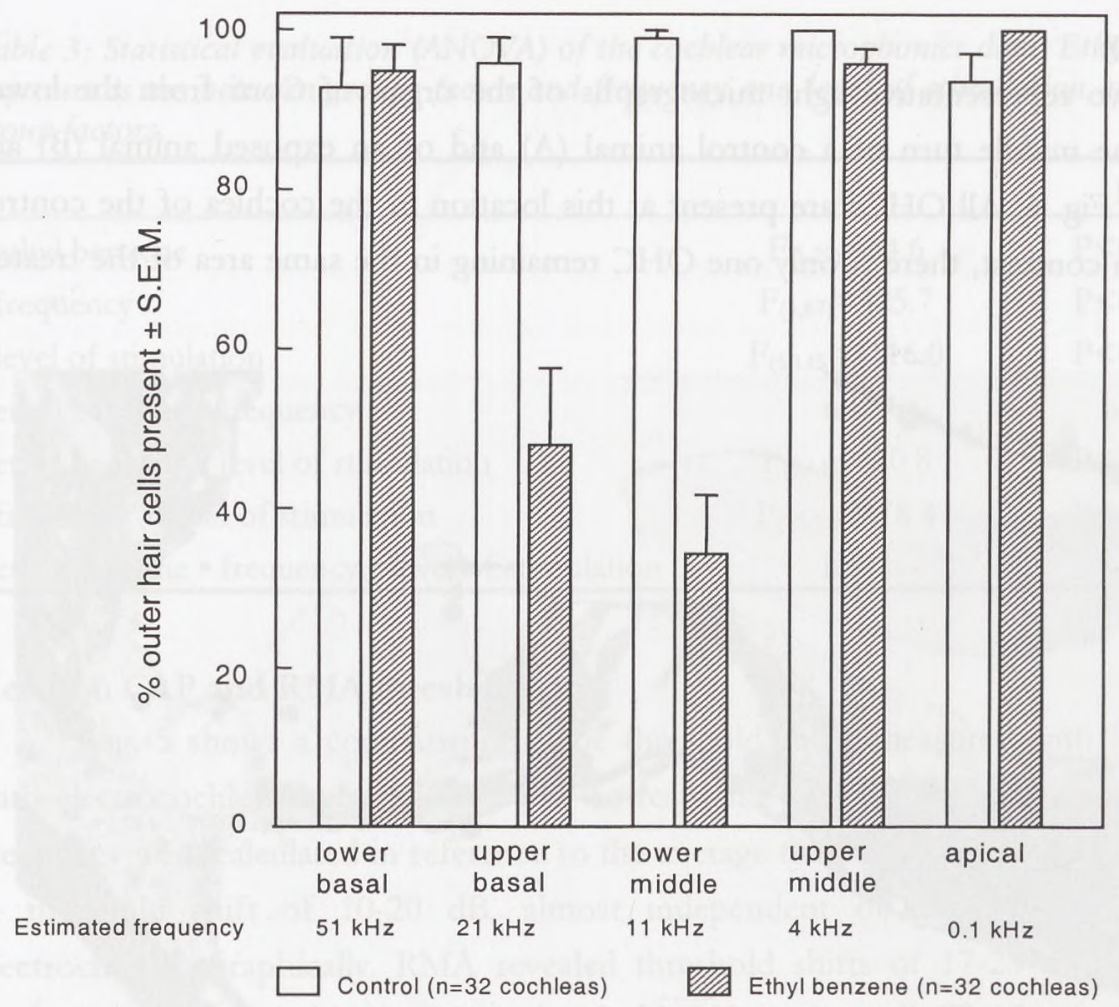

Figure 7. Outer hair cell counts at 5 positions along the basilar membrane. Data are expressed as the percentage of outer hair cells remaining ( \pm S.E.M.) per transection. Note the loss of outer hair cells in the exposed group, predominantly in the mid-frequency region.

To quantify the histological effect of ethyl benzene, the number of OHCs remaining was counted. The mean percentages of OHCs remaining at each transection in both groups are shown in Fig. 7. ANOVA showed main effects of ethyl benzene and location (in half space turns, Table 4) and a significant interaction between the two. To further evaluate the effect per location, we used Tukey's HSD post hoc test which showed that ethyl benzene induced significant OHC loss of $52.1 \pm 9.7 \%$ (mean \pm S.E.M.) in the upper part of the basal turn and $65.6 \pm 7.3 \%$ (mean \pm S.E.M.) in the lower part of the middle turn $(\mathrm{P}<0.01)$. This corresponds with the frequency range of $11-21 \mathrm{kHz}$, based on the frequency map of Müller (1991). Inner hair cells were present at $100 \%$ in both the exposed and the control group. Moreover, obvious spiral ganglion cell loss was never observed at the lightmicroscopical level. 
Table 4. Statistical evaluation (ANOVA) of the outer hair cell counts. Ethyl benzene exposure is the between-groups factor and the cochlear location (5 locations corresponding with the locations in Fig. 6) is the within-group factor.

\begin{tabular}{lll}
\hline & $F$ & $P$ \\
\hline ethyl benzene & $F_{(1,30)}=35.8$ & $P<0.01$ \\
location & $F_{(4,120)}=22.7$ & $P<0.01$ \\
ethyl benzene $\cdot$ location & $F_{(4,120)}=26.7$ & $P<0.01$ \\
\hline
\end{tabular}

\section{Discussion}

In rat, ethyl benzene exposure (800 ppm, 8 hours/day for 5 days) resulted in significantly increased RMA thresholds by about $25 \mathrm{~dB}$. Statistical analysis showed that this effect did not depend on frequency in the range of $4-24 \mathrm{kHz}$ measured. The RMA thresholds did not change between one week and four weeks post exposure. Thus, neither recovery nor further deterioration of auditory thresholds occurred within the period from one to four weeks after exposure. Similarly, the CAP data showed an auditory threshold shift (10-30 dB) which was frequency independent within the range of measurement. The thresholds in the control animals, both behaviourally and electrophysiologically (CAP), are well within published boundaries (Fay, 1988). We conclude that ethyl benzene does induce hearing loss in rats, as has been reported previously for some other organic solvents (toluene: Pryor et al., 1983; Rebert et al., 1983; Crofton et al., 1994; Johnson and Canlon, 1994a; styrene: Pryor et al., 1987; Crofton et al., 1994; trichloroethylene: Jaspers et al., 1993; Crofton and Zhao, 1993). However, the frequency independent effect of ethyl benzene, in the range of $1-24 \mathrm{kHz}$, seems inconsistent with previous reports on various other organic solvents. Numerous authors have emphasized that predominantly mid-frequency (8$20 \mathrm{kHz}$ ) hearing deficits occur in rats after exposure to organic solvents. This apparent inconsistency may be related to the level of exposure. Campo et al. (1997) exposed rats to five concentrations of toluene ( 6 hours/day, 5 days/week for 4 weeks) and tested their hearing with brainstem audiometry. The lowest concentrations applied, 1000 and $1250 \mathrm{ppm}$, induced no significant threshold shift. At $1500 \mathrm{ppm}$, the shift was relatively weak and restricted to the $20 \mathrm{kHz}$ region. The concentration of 1750 ppm induced hearing deficits at $8,10,12,16,20$ and $24 \mathrm{kHz}$. The highest concentration level of $2000 \mathrm{ppm}$ produced significant threshold shifts at all 
frequencies tested $(2-32 \mathrm{kHz})$. Thus, the frequency range affected by toluene exposure expanded with increasing concentrations of toluene. Similar results were reported for rats exposed to various concentrations of styrene and mixed xylenes (Pryor et al., 1987). Possibly, these findings for toluene, styrene and mixed xylenes can be generalised to other organic solvents, including ethyl benzene. This would imply that the concentration of ethyl benzene applied in this study was rather high. Thus, compared to other organic solvents, ethyl benzene seems to be a very potent ototoxicant. Toluene, styrene and trichloroethylene have to be dosed at higher concentrations and for a longer period of time to induce effects comparable to the ethyl benzene effects observed in this study (Pryor et al., 1987; Crofton et al., 1994; Campo et al., 1997).

The threshold shifts after ethyl benzene exposure, measured both behaviourally and electrophysiologically (CAP), were similar (Fig. 5). This result provides a clue as to the site of action of ethyl benzene. With RMA, the complete auditory pathway, both the peripheral and central part, is involved. Electrocochleography on the other hand, reflects exclusively the effects of ethyl benzene at the periphery. Because the threshold shifts from both methods were very similar, we may conclude that ethyl benzene exerts its effects predominantly in the peripheral part of the auditory pathway. The threshold shifts obtained from the CAP data are possibly even larger. These thresholds were based on the iso-response level of $1 \mu \mathrm{V}$. When the CAP data are extrapolated to lower iso-response levels, the amplitude growth curves of the exposed group and control group diverge even more. This may yield even larger threshold shifts. This gives even more support to the hypothesis that ethyl benzene effects only the peripheral part of the auditory pathway.

Recruitment, which is seen in the CAP data (Fig. 3), is generally attributed to malfunctioning of the OHCs (Patuzzi et al., 1989), suggesting an effect of ethyl benzene on the OHCs. Support for this idea can be found in our CM data (Fig. 4). The CM amplitude of the exposed group was smaller, which once again implies that the state of the OHCs was deteriorated after ethyl benzene exposure (Dallos, 1972; Dallos and Cheatham, 1976). This was confirmed by the histological analysis of the cochleas. The OHCs in the upper basal and lower middle turns have partly disappeared after ethyl benzene exposure. These two cochlear turns correspond with the mid-frequency region (Fig. 7). Our histological data are consistent with previous reports on $\mathrm{OHC}$ loss following exposure to various organic solvents. Rats exposed to 
toluene, either systemically (Sullivan et al., 1989) or by inhalation (Pryor et al., 1984; Johnson and Canlon, 1994b and Campo et al., 1997), showed OHC loss in the midfrequency region. Exposure to styrene caused similar OHC loss (Yano et al., 1992; Mäkitie, 1997). In contrast, only sporadic $\mathrm{OHC}$ loss was found after trichloroethylene exposure. Instead, a remarkable decrease in density of spiral ganglion cells was observed (Fechter et al., 1998). Obvious ganglion cell involvement was not found in this study. In this respect, the organic solvent ethyl benzene showed more resemblance to styrene and toluene than to trichloroethylene in its mode of action. Toluene $\left(\mathrm{C}_{7} \mathrm{H}_{8}\right)$, styrene $\left(\mathrm{C}_{8} \mathrm{H}_{8}\right)$ and ethyl benzene $\left(\mathrm{C}_{8} \mathrm{H}_{10}\right)$ are aromatic hydrocarbons, and all three consist of a benzene ring and a linear side chain. This in contrast to trichloroethylene $\left(\mathrm{C}_{2} \mathrm{HCl}_{2}\right)$, which is a chlorinated hydrocarbon. All four solvents mentioned above do cause identical mid-frequency hearing loss. But the mechanism of ototoxicity seems to be dissimilar. This diversity might be based on the molecular structure of the solvents.

There appears to be a discrepancy between the extensive hearing loss we have measured with RMA and electrocochleography and the focused OHC loss. A possible explanation is that subcellular damage has occurred in the remaining OHCs. This damage could be so severe that the function of the OHCs was affected, but not so serious that the OHCs had completely disappeared. The fact that the threshold shifts occurred already at one week after exposure and did not recover or not further deteriorate at 4 weeks suggests persistent sublethal damage to the OHCs. Most pathological insults either result in cell death or, especially after removal of the insult, revert to cell recovery. However, a notable example is sublethal damage to OHCs after noise damage (Engström, 1984). Further histological investigation, preferably at the electron-microscopical level might resolve this issue.

The ototoxic effect of ethyl benzene in rats occurs at a level that is only 8 times the US threshold limit value, which raises questions about the safety of human workers exposed to the compound. The TLV gives the concentration to which it is believed that daily exposure is without adverse effect. As we have shown in this study, ethyl benzene levels of 8 times TLV caused severe hearing loss. However, extrapolation from short-term animal studies at high exposure levels to long-term human exposures at lower levels requires caution (Crofton and Zhao, 1997). 
In summary, our data showed an ototoxic effect of ethyl benzene in rats with a predominantly peripheral involvement. Further analysis showed that the OHCs, especially in the mid-frequency region, are the primary targets for this compound.

\section{ACKNOWLEDGEMENTS}

This study was supported by grants of NWO-MW (grant number 9004-63085) and by the Heinsius-Houbolt Fund. The authors would like to thank R. Bulder and F. Hendriksen for their histological assistance. 


\section{ETHYL BENZENE-INDUCED OTOTOXICITY IN RATS: A DOSE-DEPENDENT MID- FREQUENCY HEARING LOSS}

Rats were exposed to ethyl benzene at 0, 300, 400 and $550 \mathrm{ppm}$ for eight hours/day for five consecutive days. Three to six weeks after the exposure auditory function was tested by measuring compound action potentials (CAP) and $2 \mathrm{f}_{1}-\mathrm{f}_{2}$ distortion product otoacoustic emissions (DPOAEs). In addition, outer hair cell (OHC) loss was quantified by histological examination. The lowest concentration ethyl benzene had no effect on any of the above measures. At $400 \mathrm{ppm}$, auditory thresholds were increased by 15 and $16 \mathrm{~dB}$ at 12 and $16 \mathrm{kHz}$, respectively and at $550 \mathrm{ppm}$ by 24,31 and $22 \mathrm{~dB}$ at 8,12 and $16 \mathrm{kHz}$, respectively. DPOAE amplitude growth with stimulus-level was affected only after 550 ppm at 5.7, 8 and $11.3 \mathrm{kHz}$. OHCs loss was found in two of the five examined locations in the cochlea. At $400 \mathrm{ppm}, 25 \%$ OHC loss was found at the 11 and $21 \mathrm{kHz}$ region. The highest concentration evoked $40 \%$ and $75 \%$ OHC loss at the 11 and $21 \mathrm{kHz}$ location, respectively. Thus, the mid-frequency region of rats is affected after exposure to relatively low concentrations of ethyl benzene ( $400-550 \mathrm{ppm})$. These results indicate that ethyl benzene is one of the most potent ototoxic organic solvents known today.

(Accepted by the Journal of Association for Research in Otorhinolaryngology; co-authors: Klis, Baretta, Muijser, Smoorenburg) 


\section{INTRODUCTION}

Several volatile organic solvents are suspected of inducing hearing impairment in humans (e.g., Morata and Dunn, 1994) and have been proven to affect hearing in animals (e.g., Crofton et al., 1994). The most widely studied agent in animal models is toluene. Originally, the location of solvent-induced hearing loss was reported to be situated in the high-frequency region of the rat cochlea, after inhalation of 1200-1400 ppm toluene for 14 hours/day, 7 days/week for 5 weeks (Pryor et al., 1983; Rebert et al., 1983). However in 1989, Sullivan and coworkers described low and mid-frequency hearing loss after oral administration of toluene. Loss of outer hair cells (OHCs) in the middle and basal turn of the cochlea accompanied this toluene-induced hearing loss. Nowadays, most authors agree on a predominantly mid-frequency hearing impairment $(8-20 \mathrm{kHz})$ after toluene exposure in rats (e.g., Crofton et al., 1994; Campo et al., 1997). Additionally, other solvents like styrene (Yano et al., 1992; Crofton et al., 1994), xylene (Crofton et al., 1994) and trichloroethylene (Crofton and Zhao, 1993; Jaspers et al., 1993; Crofton et al., 1994; Fechter et al., 1998) were found to induce midfrequency hearing loss in rats. Together with the threshold shifts in the $8-20 \mathrm{kHz}$ region, a loss of $\mathrm{OHCs}$ in the corresponding region of the basilar membrane was found as a result of toluene and styrene exposure Johnson and Canlon, 1994 and Yano et al., 1992, respectively). The affected frequency range seems to depend on the solvent concentration. The affected frequency range will spread towards lower and higher frequencies when the applied concentration is increased Johnson, 1992; Johnson and Canlon, 1994b; Lataye et al., 1999; Loquet et al., 1999).

In a previous study in our laboratory (Cappaert et al., 1999), we investigated the effect of $800 \mathrm{ppm}$ ethyl benzene on hearing in rats. This concentration was moderate compared to ototoxic concentrations of other organic solvents. However, rats exposed to $800 \mathrm{ppm}$ ethyl benzene ( 8 hours/day, 5 days) showed a severe hearing loss of $20-25 \mathrm{~dB}$ in the entire investigated $1-24 \mathrm{kHz}$ region. Although the OHC loss was large in the mid-frequency region, the finding of a flat hearing loss was not in line with the results for other organic solvents. In the present study we have exposed three groups of rats to three lower concentrations of ethyl benzene (300, 400 and $550 \mathrm{ppm})$ in order to establish a dose-response relation for ethyl benzene-induced hearing loss and to determine the extent of the affected frequency region as a function of the 
concentration of ethyl benzene. The auditory function of the animals was tested three to six weeks after the last exposure, by measuring distortion product otoacoustic emissions (DPOAEs) and compound action potentials (CAPs). In addition, we examined the number of OHCs present in the organ of Corti after the ethyl benzene exposure.

\section{METHODS}

\section{Animal care and experimental design}

We used $32 \mathrm{Wag} /$ Rij rats (obtained from Charles River, Germany) with an average weight of $200 \mathrm{~g}$ at the beginning of the experiment. The rats were grouphoused in wire-mesh cages (four per cage). Food and water were available ad libitum. Lights were on from 7:00 a.m. to 7:00 p.m. The animals' weight was recorded weekly. Care and use of the animals reported on in this paper were approved by the Animal Care and Use Committee of the Faculty of Medicine, University Medical Center Utrecht, under number DEC-GNK-95011.

The rats were exposed to either $0,300,400$ or $550 \mathrm{ppm}$ ethyl benzene (eight rats per group) for 8 hours/day for 5 consecutive days. During exposure, the animals were placed in wire mesh cages in a stainless steel exposure chamber (Hazleton Systems Inc., Aberdeen, UK). The exposure started at 8:30 a.m. Ethyl benzene (Merck, Darmstadt, Germany, 99\% pure) was evaporated at an elevated temperature and the saturated gas was injected into the air supply system that operated at 25 $\mathrm{m}^{3} / \mathrm{hr}$. A dual gas monitor (Brüel \& Kjær, Copenhagen, Denmark, Type 3426) continuously monitored the ethyl benzene concentration in the chamber. The concentration was kept within $5 \%$ of the desired concentration. The overall ambient noise-level did not exceed $66 \mathrm{~dB}$ SPL. Possible differences in weight gain between the ethyl benzene-treated animals and that of the control animals were tested with a t-test. Measurements of DPOAE, CAP and morphological examination of the organ of Corti were performed in the period between three to six weeks after the last ethyl benzene exposure. The rats were anaesthetised during surgery and during the DPOAE and CAP measurements with an intramuscular injection of Thalamonal $(0.1 \mathrm{ml} / 100 \mathrm{~g}$ body weight; Thalamonal contains $2.5 \mathrm{mg} / \mathrm{ml}$ droperidol and $0.05 \mathrm{mg} / \mathrm{ml}$ fentanyl). This was followed by ventilation with a gas mixture containing $33 \% \mathrm{O}_{2}, 66 \% \mathrm{~N}_{2} \mathrm{O}$ 
and $1 \%$ Halothane through a trachea cannula. Heart frequency was monitored and body temperature was kept at $38^{\circ} \mathrm{C}$.

\section{Distortion product otoacoustic emissions}

A host computer, with signal processing board (AP2) and modular audiometry system (Tucker Davis Technologies, Gainesville, FL, USA) controlled stimulus generation and acquisition of DPOAE. This system was controlled by custom-made software. Two primaries ( $f_{1}$ and $f_{2}$ ), both $240 \mathrm{~ms}$ in duration, were generated by the computer and sent to two separate D/A converters. Subsequently, the primaries were fed through low-pass cut-off filters $(36 \mathrm{kHz})$, attenuators and headphone buffers. The resulting stimuli were delivered to the ear canal through an ER-10C probe system (Etymotic Research, Elk Grove Village, IL, USA), incorporating two speakers and one low-noise microphone. The microphone signal was amplified (40 dB) and A/D converted. Data acquisition started $100 \mathrm{~ms}$ after the onset of the primaries, to avoid start-up transients. The primaries and the response were both sampled at a rate of 66.7 $\mathrm{kHz}$. The recorded signals were time-averaged, windowed (Hanning shaped) and transformed by fast Fourier analysis. To calibrate the microphone of the probe system in $\mathrm{dB}$ SPL, a microphone correction table was generated, using the frequencyresponse of the ER-10C microphone provided by the manufacturer. At the beginning of each measurement, the probe was positioned in the ear canal and the primaries were adjusted per ear. According to the table, $f_{1}$ and $f_{2}$ were presented at $60 \mathrm{~dB}$ SPL and the responses were measured via the ER-10C microphone. If the measured amplitudes deviated from the target amplitude, the attenuator settings were adjusted. $2 \mathrm{f}_{1}-\mathrm{f}_{2}$ amplitude growth functions with stimulus level were obtained for the primary $\mathrm{f}_{2}$ frequencies of $4,5.7,8,11.3,16$ and $22.6 \mathrm{kHz}$. The $f_{1} / f_{2}$ ratio was 1.25 . The level of the $f_{1}$ primary $\left(L_{1}\right)$ was increased in $5 \mathrm{~dB}$ steps from 30 to $80 \mathrm{~dB}$ SPL and the level of the $\mathrm{f}_{2}$ primary $\left(\mathrm{L}_{2}\right)$ was $10 \mathrm{~dB}$ lower than $\mathrm{L}_{1}\left(\mathrm{~L}_{2}=\mathrm{L}_{1}-10\right)$. Each pair of primaries was presented 5 times. DPOAE growth curves with stimulus levels were obtained from both ears. The level of the noise floor at the location of the $2 f_{1}-f_{2}$ was measured immediately before the $2 f_{1}-f_{2}$ determination, with the same procedure and using the same recording and stimulus set-up, but with the $f_{1}$ and $f_{2}$ switched off.

Analysis of variance (ANOVA) was performed on the DPOAE growth curves averaged over left and right ears. Ethyl benzene exposure was a between-subjects 
factor and frequency and level of stimulation were within-subject factors. Post hoc Tukey's HSD test was used to further examine the significance of the differences.

\section{Compound action potentials}

CAP measurement was performed immediately after DPOAE measurement. Electrocochleographic techniques were described elsewhere for guinea pigs (Stengs et al. 1997) and are summarized here. Auditory evoked responses were recorded differentially with a silver ball electrode at the apex of the cochlea. Trains of $8 \mathrm{~ms}$ tone bursts of $1,2,4,8,12,16$ and $24 \mathrm{kHz}$ were presented, with cosine-shaped rise and fall time of $1 \mathrm{~ms}$, except at $1 \mathrm{kHz}(2 \mathrm{~ms})$ and at $2 \mathrm{kHz}(1.5 \mathrm{~ms})$. The inter-stimulus interval was $99 \mathrm{~ms}$. The responses were amplified and band-pass filtered ( $-12 \mathrm{~dB} /$ octave, -3 $\mathrm{dB}$ at $1 \mathrm{~Hz}$ and $10 \mathrm{kHz}$ ). The averaged responses were saved for off-line analysis. CAP amplitude was defined as the difference between the first negative peak and the summating potential in the electrophysiologic response. As the objective threshold we used an iso-response-criterion at a $1 \mu \mathrm{V}$ level. CAP data was logarithmically transformed before analysis to improve homogeneity of variance. Subsequently, data from left and right ears were averaged per stimulus condition. ANOVA was performed as for the DPOAE data.

\section{Histology}

Immediately after electrocochleography, the cochleas were fixed by transcardial perfusion with a chilled tri-aldehyde fixative (3\% glutaraldehyde, $2 \%$ formaldehyde, $1 \%$ acrolein, $2.5 \%$ dimethylsulfoxide in $0.1 \mathrm{M}$ sodium cacodylate buffer ( $\mathrm{pH} 7.4)$ ). The harvested cochleas were immersed in the same fixative at $4^{\circ} \mathrm{C}$ for one night. The cochleas were further processed according to the routine method used for guinea pigs (De Groot et al. 1987). In mid-modiolar sections $(1 \mu \mathrm{m})$ of the rat cochlea, the organ of Corti was examined at five locations, separated by a half-turn distance. Hair cell counts were performed at each of these five locations in both the left and the right cochlea, using 5-6 serial sections. If a hair cell is not seen in these 5-6 serial sections, it can be reliably assumed that there is no cell present. OHC loss was expressed as the percentage of remaining OHCs per cross-sectioned half turn, relative to the expected number of OHCs. Two investigators, independently of one other, performed all OHC counts in a single-blind fashion. An estimate was made of the characteristic 
frequencies of the locations along the basilar membrane at which the OHCs were counted (Müller, 1991).

The results for the left and right cochleas were averaged per location. ANOVA was used for statistical evaluation of the $\mathrm{OHC}$ counts. Ethyl benzene exposure was a between-subjects factor, cochlear location a within-subject factor. The significance of differences was tested post hoc using Tukey's HSD test.

\section{RESULTS}

\section{Animals}

The rats exhibited no overt signs of ill health following ethyl benzene exposure. The weight of the exposed rats did not differ from the weight of the control animals at the end of the exposure period for all concentrations of ethyl benzene applied.

\section{Distortion product otoacoustic emissions}

The effects of ethyl benzene on the mean $2 \mathrm{f}_{1}-\mathrm{f}_{2}$ amplitude growth curves as a function of $L_{1}$ at 8 and $11.3 \mathrm{kHz}\left(f_{2}\right)$ are depicted in Fig. 1.
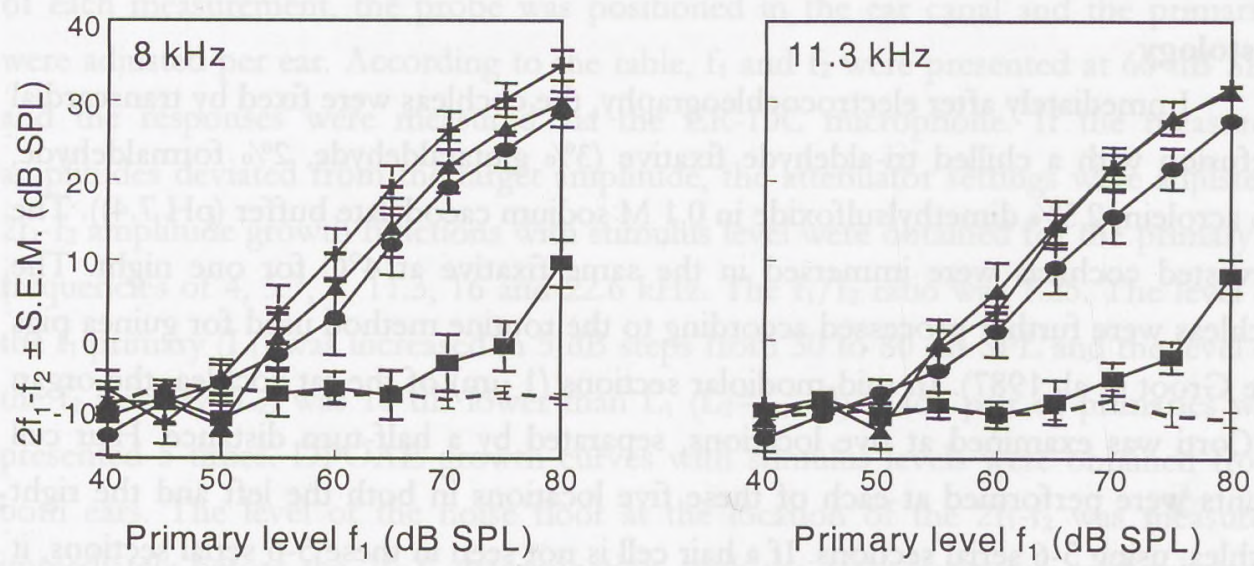

Figure 1. Effect of ethyl benzene exposure on $2 f_{1}-f_{2} D P O A E$ amplitude growth curves with stimulus-level obtained with $f_{2}$ at 8 and $11.3 \mathrm{kHz}$. Shown are the growth curves obtained after control conditions (+), 300 ppm ethyl benzene $(\mathbf{\Delta}), 400$ ppm ethyl benzene $(\bullet)$ and 550 ppm ethyl benzene ( $\mathbf{\square}$. The dashed line is the noise floor. 
The DPOAE growth curves were affected by ethyl benzene applied at $550 \mathrm{ppm}$. Ethyl benzene applied at 300 and $400 \mathrm{ppm}$ did not induce decreased DPOAE growth curve at any frequency. This was confirmed by ANOVA (performed on all tested frequencies), that showed a main effect of ethyl benzene concentration $\left(F_{(3,26)}=10.36\right.$, $\mathrm{P}<0.01)$. Also a frequency effect $\left(\mathrm{F}_{(5,130)}=117.9, \mathrm{P}<0.01\right)$ and an interaction between solvent concentration and frequency $\left(\mathrm{F}_{(8,208)}=437.8, \mathrm{P}<0.01\right)$ was found. This interaction was further pursued by ANOVA per frequency and subsequent post hoc tests. Effects of ethyl benzene were detected at $5.7 \mathrm{kHz}$ (not shown, $\mathrm{F}_{(3,26)}=5.7$; $\mathrm{P}<0.01), 8 \mathrm{kHz}\left(\mathrm{F}_{(3,26)}=18.5 ; \mathrm{P}<0.01\right)$ and $11.3 \mathrm{kHz}\left(\mathrm{F}_{(3,26)}=19.9 ; \mathrm{P}<0.01\right)$. Post hoc analysis (Tukey HSD) on ethyl benzene showed significantly decreased growth curves after $550 \mathrm{ppm}$ ethyl benzene at $5.7(\mathrm{P}<0.05), 8$ and 11.3 (both $\mathrm{P}<0.01$ ). Other frequencies remained unaffected.

A DPOAE "threshold" was defined as the stimulus level $\left(\mathrm{L}_{1}\right)$ required to evoke a $0 \mathrm{~dB}$ SPL $2 \mathrm{f}_{1}-\mathrm{f}_{2}$ DPOAE. This DPOAE iso-response criterion was chosen because this level was well above the noise floor at all tested frequencies and thus could be determined unambiguously. In control animals, these levels were typically 20 $\mathrm{dB}$ above $\mathrm{CAP} 1 \mu \mathrm{V}$ iso-response levels.

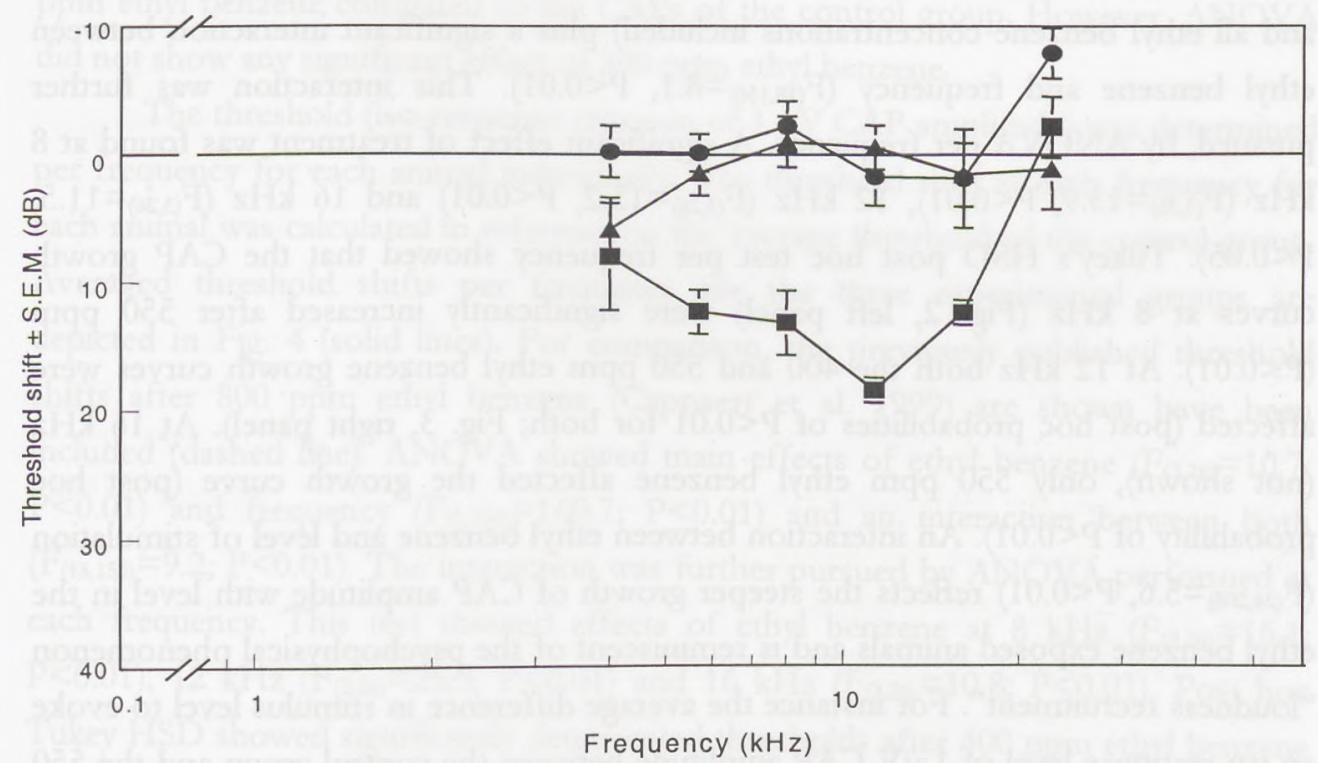

Figure 2. Threshold shifts (based on a $0 \mathrm{~dB}$ SPL $2 f_{1}-f_{2}$ DPOAE iso-response criterion) as a result of 300 ppm ethyl benzene (山), 400 ppm ethyl benzene $(\bullet)$ and 550 ppm ethyl benzene (घ) exposure. Threshold shifts are plotted downward. 
Threshold shifts in each exposed animal at each frequency were determined in reference to the average threshold level in the control group. The average threshold shifts in the three exposed groups are shown in Fig. 2.

ANOVA showed a main effect of ethyl benzene concentration $\left(F_{(3,26)}=1013.2\right.$, $\mathrm{P}<0.01)$ and frequency $\left(\mathrm{F}_{(5,130)}=1608.3, \mathrm{P}<0.01\right)$, and an interaction between these factors $\left(\mathrm{F}_{(15,130)}=106.8, \mathrm{P}<0.01\right)$. The interaction was further pursued by ANOVA performed at each frequency. This test showed effects of ethyl benzene at $5.7 \mathrm{kHz}$ $\left(\mathrm{F}_{(3,26)}=282.6, \mathrm{P}<0.01\right), 8 \mathrm{kHz}\left(\mathrm{F}_{(3,26)}=405.7, \mathrm{P}<0.01\right), 11.3 \mathrm{kHz}\left(\mathrm{F}_{(3,26)}=642.4, \mathrm{P}<0.01\right)$ and $16 \mathrm{kHz} \quad\left(\mathrm{F}_{(3,26)}=249.3, \mathrm{P}<0.01\right)$. Post hoc Tukey HSD showed significantly increased stimulus levels after $550 \mathrm{ppm}$ ethyl benzene at $5.7 \mathrm{kHz}(\mathrm{P}<0.01), 8 \mathrm{kHz}$ $(\mathrm{P}<0.01), 11.3 \mathrm{kHz}(\mathrm{P}<0.01)$ and $16 \mathrm{kHz}(\mathrm{P}<0.05)$.

\section{Compound action potentials}

Fig. 3 depicts mean CAP amplitude growth curves as a function of stimulus level at 8 and $12 \mathrm{kHz}$ (all groups included). Main effects of ethyl benzene concentration $\left(\mathrm{F}_{(3,26)}=8.2, \mathrm{P}<0.01\right)$, frequency $\left(\mathrm{F}_{(6,156)}=231.7, \mathrm{P}<0.01\right)$ and level of stimulation $\left(\mathrm{F}_{(8,208)}=1561.2, \mathrm{P}<0.01\right)$ were found with ANOVA (all tested frequencies and all ethyl benzene concentrations included) plus a significant interaction between ethyl benzene and frequency $\left(\mathrm{F}_{(18,156)}=8.1, \mathrm{P}<0.01\right)$. This interaction was further pursued, by ANOVA per frequency. A significant effect of treatment was found at 8 $\mathrm{kHz}\left(\mathrm{F}_{(3,26)}=13.9, \mathrm{P}<0.01\right), 12 \mathrm{kHz}\left(\mathrm{F}_{(3,26)}=15.2, \mathrm{P}<0.01\right)$ and $16 \mathrm{kHz}\left(\mathrm{F}_{(3,26)}=11.5\right.$, $\mathrm{P}<0.05)$. Tukey's HSD post hoc test per frequency showed that the CAP growth curves at $8 \mathrm{kHz}$ (Fig. 2, left panel) were significantly increased after $550 \mathrm{ppm}$ $(\mathrm{P}<0.01)$. At $12 \mathrm{kHz}$ both the 400 and $550 \mathrm{ppm}$ ethyl benzene growth curves were affected (post hoc probabilities of $\mathrm{P}<0.01$ for both; Fig. 3, right panel). At $16 \mathrm{kHz}$ (not shown), only $550 \mathrm{ppm}$ ethyl benzene affected the growth curve (post hoc probability of $\mathrm{P}<0.01)$. An interaction between ethyl benzene and level of stimulation $\left(F_{(24,208)}=5.6, P<0.01\right)$ reflects the steeper growth of CAP amplitude with level in the ethyl benzene exposed animals and is reminiscent of the psychophysical phenomenon "loudness recruitment". For instance the average difference in stimulus level to evoke an iso-response level of $1 \mu \mathrm{V} \mathrm{CAP}$ amplitude between the control group and the 550 ppm ethyl benzene group at $12 \mathrm{kHz}$, was $30.6 \pm 2 \mathrm{~dB}$. At $10 \mu \mathrm{V}$ the difference was decreased to $24.3 \pm 3.3 \mathrm{~dB}$. Full recruitment (no difference between the CAP amplitude 

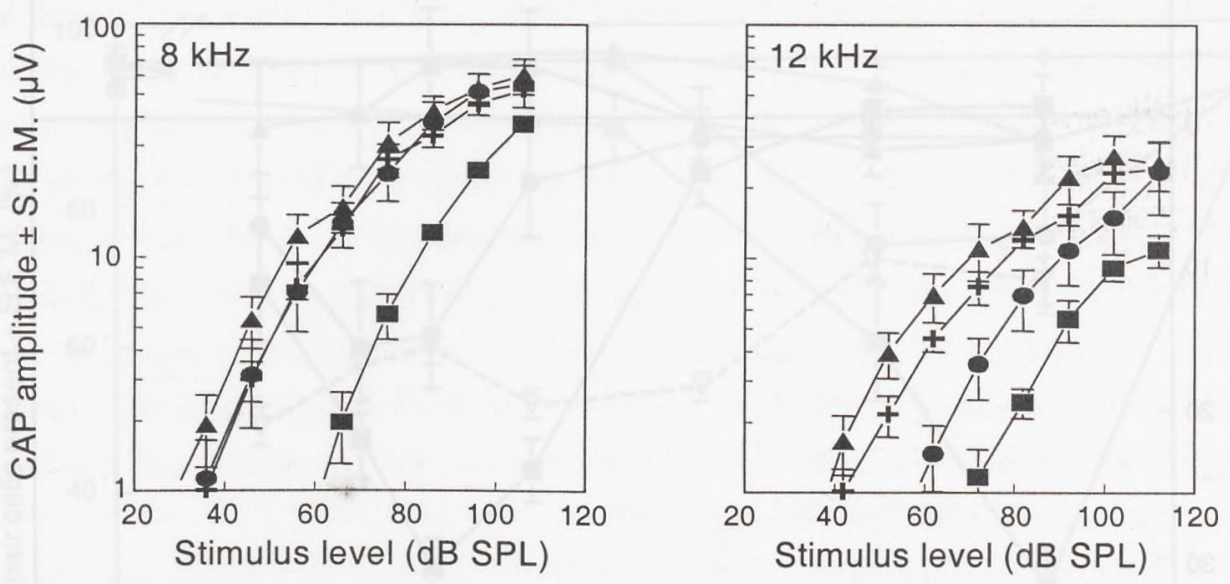

Figure 3. CAP amplitude growth curves with stimulus level for 8 and $12 \mathrm{kHz}$ after exposure to control conditions (+), 300 ppm ethyl benzene $(\mathbf{\Delta}), 400$ ppm ethyl benzene (•) and

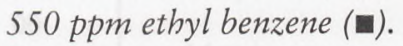

of the control and the exposed group at high stimulus levels) was not reached. Both panels of Fig. 3 show slightly larger CAPs as a function of stimulus level after 300 ppm ethyl benzene compared to the CAPs of the control group. However, ANOVA did not show any significant effect of $300 \mathrm{ppm}$ ethyl benzene.

The threshold (iso-response criterion of $1 \mu \mathrm{V}$ CAP amplitude) was determined per frequency for each animal individually. The threshold shift at each frequency for each animal was calculated in reference to the average threshold of the control group. Averaged threshold shifts per frequency for the three experimental groups are depicted in Fig. 4 (solid lines). For comparison, the previously published threshold shifts after 800 ppm ethyl benzene (Cappaert et al. 1999) are shown have been included (dashed line). ANOVA showed main effects of ethyl benzene $\left(\mathrm{F}_{(3,26)}=10.7\right.$; $\mathrm{P}<0.01)$ and frequency $\left(\mathrm{F}_{(0,156)}=160.7 ; \mathrm{P}<0.01\right)$ and an interaction between both $\left(\mathrm{F}_{(18,156)}=9.2 ; \mathrm{P}<0.01\right)$. The interaction was further pursued by ANOVA performed at each frequency. This test showed effects of ethyl benzene at $8 \mathrm{kHz}\left(\mathrm{F}_{(3,26)}=16.1\right.$; $\mathrm{P}<0.01), 12 \mathrm{kHz}\left(\mathrm{F}_{(3,26)}=25.9 ; \mathrm{P}<0.01\right)$ and $16 \mathrm{kHz}\left(\mathrm{F}_{(3,26)}=10.8 ; \mathrm{P}<0.01\right)$. Post hoc Tukey HSD showed significantly deteriorated thresholds after $400 \mathrm{ppm}$ ethyl benzene at 12 and $16 \mathrm{kHz}(\mathrm{P}<0.05)$. The highest concentration ethyl benzene in the present study $(550 \mathrm{ppm})$ affected an even broader range of frequencies: significant threshold 


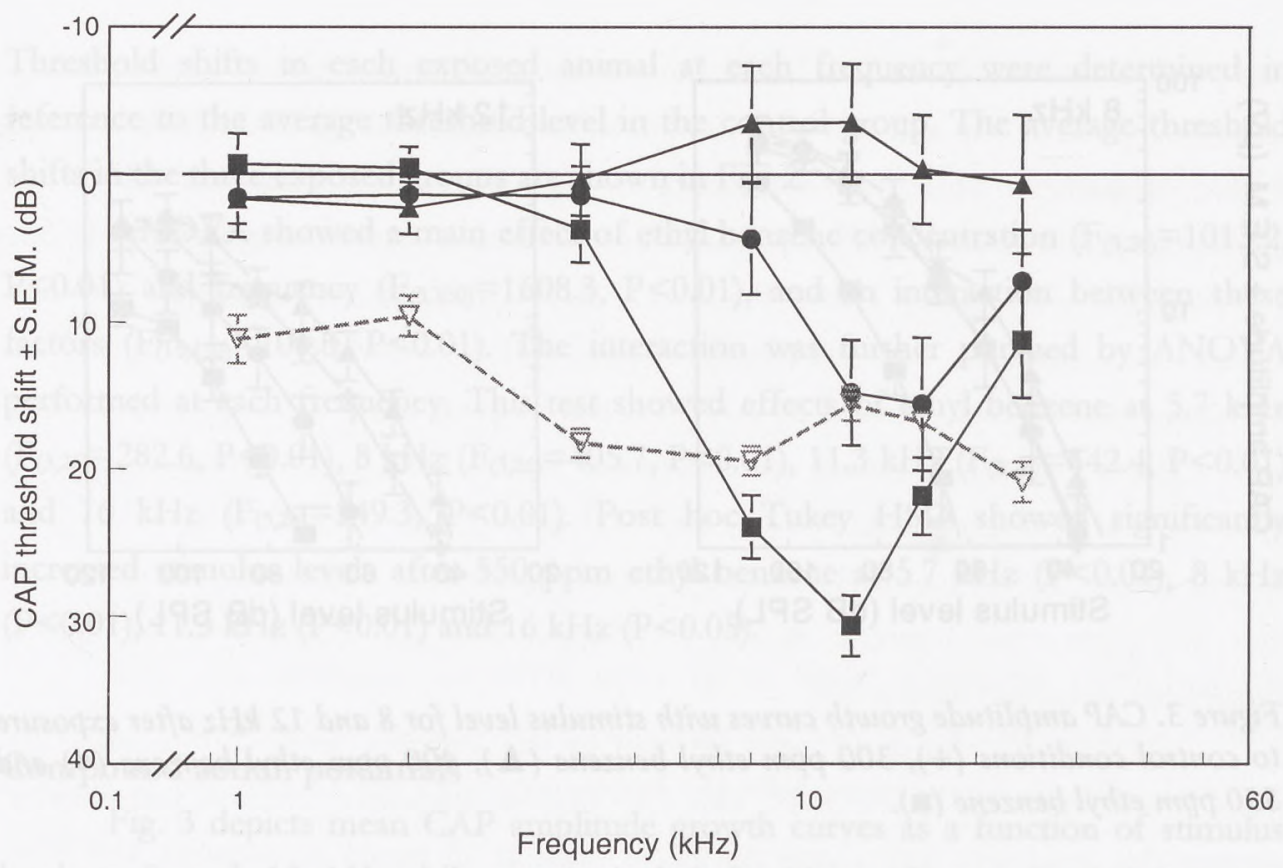

Figure 4. Threshold shifts (based on a CAP $1 \mu \mathrm{V}$ iso-response criterion) as a result of 300

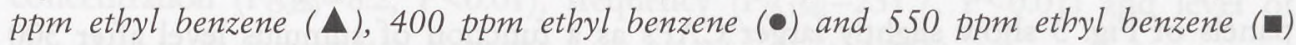
exposure. Threshold shifts are plotted downward. Threshold shifts as a result of 800 ppm ethyl benzene exposure (Cappaert et al. 1999) are added (dashed line).

shifts were detected at 8,12 and $16 \mathrm{kHz}(\mathrm{P}<0.01)$. In contrast, exposure at $300 \mathrm{ppm}$ ethyl benzene did not significantly affect the auditory thresholds.

\section{Histology}

OHC counts are shown in Fig. 5. The mean percentage of OHCs present is shown for each location examined. OHC loss was most severe in the 550 ppm group. $60 \%$ and $25 \%$ of the OHC remained at the 11 and $21 \mathrm{kHz}$ location, respectively. The effects at $400 \mathrm{ppm}$ were less severe than the effects at $550 \mathrm{ppm}$. About $75 \%$ of the OHCs were present after $400 \mathrm{ppm}$ ethyl benzene at both the 11 and $21 \mathrm{kHz}$ location. A small OHC loss was found in the $21 \mathrm{kHz}$ region of the $300 \mathrm{ppm}$ group ( $12 \%$ loss). The control group showed no OHC loss. Statistical analysis (ANOVA) showed main effects of ethyl benzene concentration $\left(\mathrm{F}_{(3,27)}=28.8, \quad \mathrm{P}<0.01\right)$ and location $\left(F_{(4,108)}=38.1, P<0.01\right)$ and a significant interaction between the two $\left(F_{(12,108)}=13.8\right.$, $\mathrm{P}<0.01)$. A Tukey's HSD post hoc test was used to further evaluate the effect of ethyl 


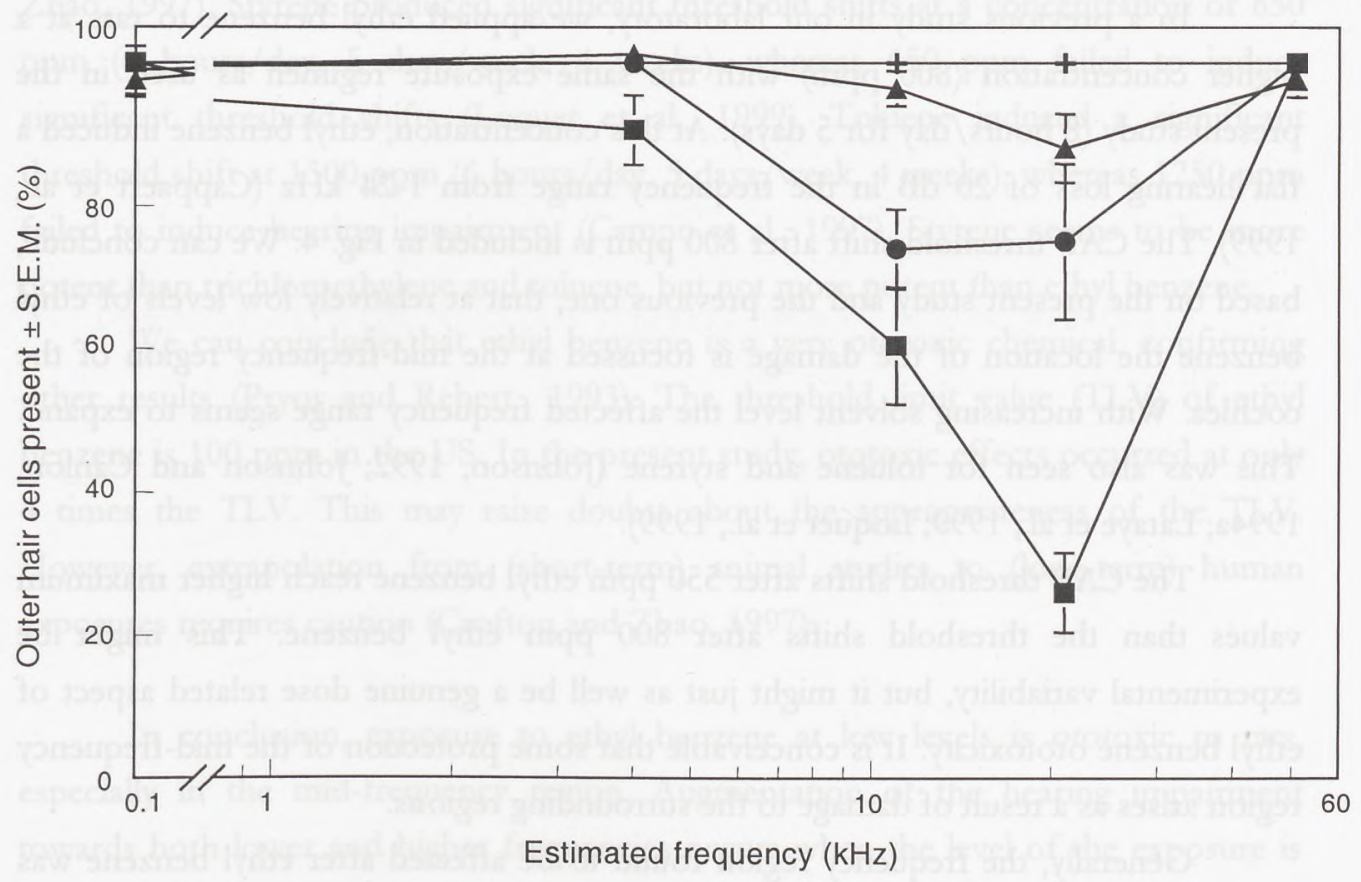

Figure 5. Percentage of $\mathrm{OHC}$ present at 5 locations in the cochlea after ethyl benzene exposure (control conditions (+), 300 ppm ethyl benzene $(\mathbf{\Delta}), 400$ ppm ethyl benzene $(\bullet)$ and $550 \mathrm{ppm}$ ethyl benzene (-)). The locations are presented as estimates of the characteristic frequencies at the locations examined (see Material and Methods section).

benzene per location. Significant effects of $550 \mathrm{ppm}$ ethyl benzene were found at the 11 and $21 \mathrm{kHz}$ location (both $\mathrm{P}<0.01$ ). A significant $\mathrm{OHC}$ loss after $400 \mathrm{ppm}$ was found only at the $11 \mathrm{kHz}$ location $(\mathrm{P}<0.01)$.

\section{Discussion}

The auditory periphery of the rat is clearly affected after 400 and $550 \mathrm{ppm}$ ethyl benzene. Threshold shifts and $\mathrm{OHC}$ loss are located in the $5.7-16 \mathrm{kHz}$ region. This indicates a mid-frequency effect of this agent, as was observed in other studies for organic solvents like toluene, xylene, styrene and trichloroethylene (Yano et al., 1992; Crofton and Zhao, 1993; Jaspers et al., 1993; Crofton et al., 1994; Fechter et al., 1998). 
In a previous study in our laboratory, we applied ethyl benzene to rats at a higher concentration (800 ppm) with the same exposure regimen as used in the present study ( 8 hours/day for 5 days). At this concentration, ethyl benzene induced a flat hearing loss of $20 \mathrm{~dB}$ in the frequency range from 1-24 kHz (Cappaert et al., 1999). The CAP threshold shift after $800 \mathrm{ppm}$ is included in Fig. 4. We can conclude, based on the present study and the previous one, that at relatively low levels of ethyl benzene the location of the damage is focussed at the mid-frequency region of the cochlea. With increasing solvent level the affected frequency range seems to expand. This was also seen for toluene and styrene Johnson, 1992; Johnson and Canlon, 1994a; Lataye et al., 1999; Loquet et al., 1999).

The CAP threshold shifts after 550 ppm ethyl benzene reach higher maximum values than the threshold shifts after $800 \mathrm{ppm}$ ethyl benzene. This might be experimental variability, but it might just as well be a genuine dose related aspect of ethyl benzene ototoxicity. It is conceivable that some protection of the mid-frequency region arises as a result of damage to the surrounding regions.

Generally, the frequency region found to be affected after ethyl benzene was comparable, irrespective of the method used for evaluation. Thus, the highest concentration of ethyl benzene applied in this study (550 ppm) decreased the $2 \mathrm{f}_{1}-\mathrm{f}_{2}$ amplitude growth curves at $5.7,8$ and $11.3 \mathrm{kHz}$, it induced CAP threshold shifts at 8 , 12 and $16 \mathrm{kHz}$ and $\mathrm{OHCs}$ were affected at the 11 and $21 \mathrm{kHz}$ location. However, at 400 ppm ethyl benzene, CAP threshold shifts were in line with OHC loss, but not with DPOAE measurements. The latter seem to be less sensitive than CAP measurements and OHC counts. This is in agreement with observations reported by Subramaniam et al. $(1994,1995)$. They reported normal DPOAE growth curves in chinchilla's with a hearing loss of approximately 25 to $30 \mathrm{~dB}$ and clear $\mathrm{OHC}$ pathology.

The minimum concentration of ethyl benzene required to induce significant threshold shifts was 400 ppm, applied for 8 hours/day during five days, whereas 300 ppm ethyl benzene did not affect the auditory system significantly (except for some minor OHC loss, which might be enhanced when combined with other ototoxic chemicals or noise (Johnson et al., 1988; Lataye and Campo, 1997; Lataye et al., 2000). Compared to the available data for other ototoxic solvents, ethyl benzene affects the auditory periphery in rats at a rather low value. The lowest ototoxic concentration of trichloroethylene ( 6 hours/day, 5 days) was between 1600 and 2400 ppm (Crofton and 
Zhao, 1997). Styrene produced significant threshold shifts at a concentration of 850 $\mathrm{ppm}$ (6 hours/day, 5 days/week, 4 weeks), whereas $650 \mathrm{ppm}$ failed to induce significant threshold shifts (Loquet et al., 1999). Toluene induced a significant threshold shift at $1500 \mathrm{ppm}$ (6 hours/day, 5 days/week, 4 weeks), whereas $1250 \mathrm{ppm}$ failed to induce hearing impairment (Campo et al., 1997). Styrene seems to be more potent than trichloroethylene and toluene, but not more potent than ethyl benzene.

We can conclude that ethyl benzene is a very ototoxic chemical, confirming other results (Pryor and Rebert., 1993). The threshold limit value (TLV) of ethyl benzene is $100 \mathrm{ppm}$ in the US. In the present study, ototoxic effects occurred at only 4 times the TLV. This may raise doubts about the appropriateness of the TLV. However, extrapolation from (short-term) animal studies to (long-term) human exposures requires caution (Crofton and Zhao, 1997).

In conclusion, exposure to ethyl benzene at low levels is ototoxic to rats, especially in the mid-frequency region. Augmentation of the hearing impairment towards both lower and higher frequencies occurs when the level of the exposure is increased.

\section{ACKNOWLEDGEMENTS}

The authors wish to thank R. Bulder and F. Hendriksen for their histological assistance and E. Duistermaat for his technical assistance with the ethyl benzene exposures. This study was supported by the Netherlands Organization for Scientific Research, Council of Medical Sciences (NWO-MW, grant number 9004-63-085) and by the Heinsius-Houbolt Fund. 


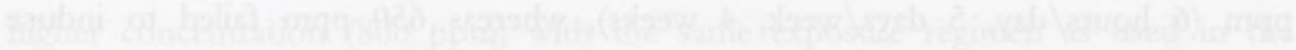

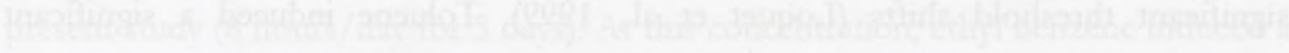

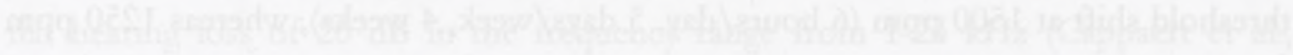

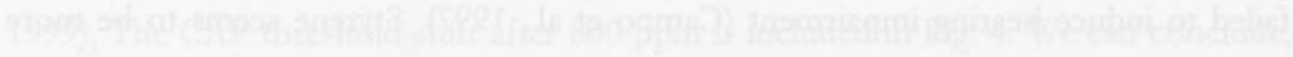

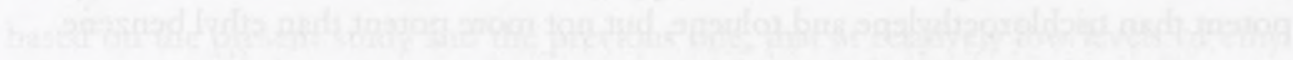

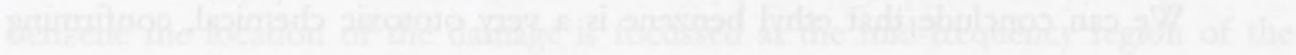

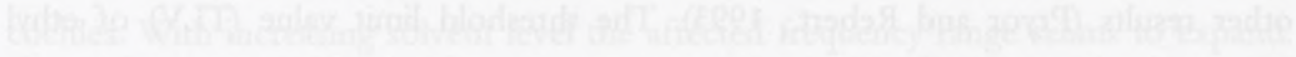

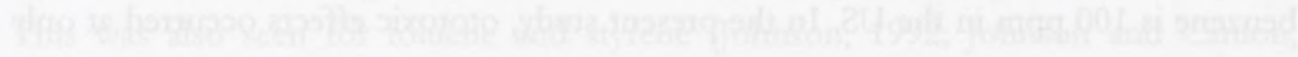

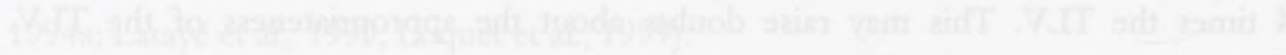

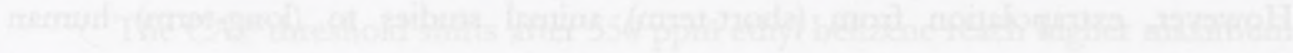

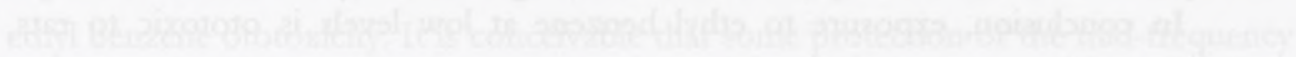

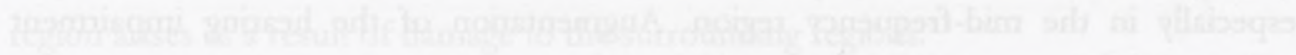

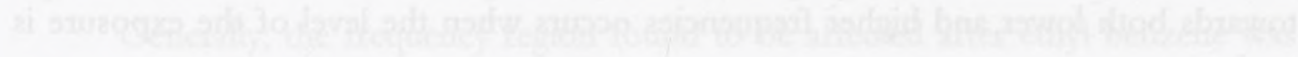

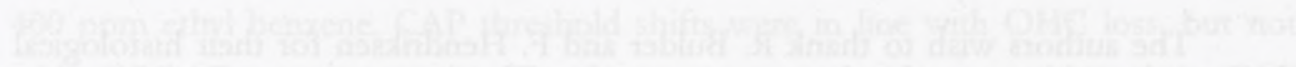

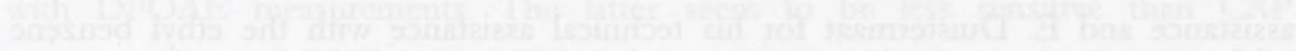

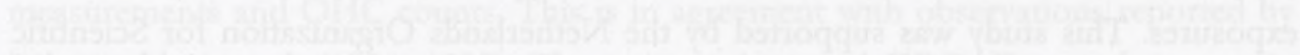

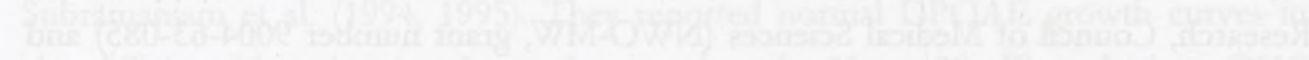

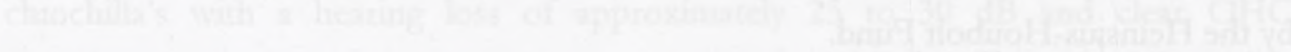




\section{NOISE-INDUCED HEARING LOSS IN RATS}

The effect of noise exposure on the auditory system is well known from animal studies. However, most of the studies concern short-term exposure conditions. The purpose of the present research was to find the dose-effect curve for hearing loss in rats following 5 days of noise exposure. Three groups of eight Wag/Rij rats were exposed to broad-band noise at levels of 90,100 and $110 \mathrm{~dB}$ SPL for 8 hours/day and 5 consecutive days. An additional group of eight rats served as the control group. Between three and seven weeks after the exposure, hearing was tested by electrochochleography (CAPs) and distortion product otoacoustic emissions (DPOAEs). Subsequently, the cochleas were morphologically examined. Only the highest two exposure levels affected hearing. The DPOAE growth curves at 4, 8 and $16 \mathrm{kHz}$ and the CAP growth curves at 4, 8, 12, 16 and $24 \mathrm{kHz}$ were affected after the $110 \mathrm{~dB}$ SPL broad-band noise. After the $100 \mathrm{~dB}$ SPL noise, only the $12 \mathrm{kHz}$ CAP growth curve was affected. At the light-microscopic level, OHC damage was not detected in this study.

(Accepted by Noise \& Health; co-authors: Klis, Muijser, Kulig, Smoorenburg) 


\section{INTRODUCTION}

Noise-induced hearing loss (NIHL) is a significant occupational health problem in industrialised countries (Alberti, 1998). NIHL has been studied extensively in both humans and animals (see: Saunders et al., 1985; Borg et al., 1995; Salvi et al., 1995 for review). A complex interaction between intensity, duration, spectral energy distribution and temporal patterns of the sound determines the amount of hearing loss, the frequencies at which hearing is affected and reversibility of the hearing loss (see: Borg et al., 1995; Salvi et al., 1995 for review). At present, at least two mechanisms have been proposed for NIHL: mechanical injuries to the receptor cells induced by excessive movement of the cochlear partition (Saunders et al., 1985) and damage due to metabolic exhaustion resulting in distortion of the homeostasis of the organ of Corti (Slepecky, 1986). Probably, these two mechanisms both contribute to NIHL.

Several studies have been performed with rats exposed to noise alone for a period longer than one day. However, these studies used different strains of rats, different exposure paradigms and different types of noise spectra (Borg, 1982a,b; Engström, 1984, Johnson et al., 1988; 1990; Lataye and Campo, 1997; Sullivan and Conolly, 1988). The purpose of the present study was to establish the relationship between the level of broad-band noise ( 8 hours/day for 5 consecutive days) and the amount of functional hearing loss together with the accompanying morphological changes in the organ of Corti of the Wag/Rij rat. With these data, it is possible to determine a dose-effect curve to quantify the damage.

This dose-effect curve is important with regard to the issue of combined exposure to noise and ototoxic chemicals. Exposure to various chemical compounds, like aminoglycosides, cisplatin, loop diuretics, salicylate and organic solvents has been proven to impair hearing. Moreover, these chemical compounds seem to potentiate NIHL (see Boettcher et al., 1987 for review). To investigate the combined effects of noise and ototoxic compounds, it is important to expose groups of experimental animals both to the noise and the ototoxic compound alone and to the combinations of the two, at moderate levels of the noise and chemicals. Thus, the purpose of this study is 1) to provide a baseline for the effects of noise on hearing in rats, which will be used in ongoing studies on combined exposures in our laboratory, and 2) to add to 
the rather limited information we have on the effects of noise on hearing in this species.

\section{MATERIALS AND METHODS}

\section{Animals}

Male Wag/Rij rats, with an average weight of $200 \mathrm{~g}$ at the beginning of the experiment, were obtained from Charles River (Germany). Animals were grouphoused in wire mesh cages (four per cage). Food and water were available ad libitum. Lights were on from 7:00 a.m. to 7:00 p.m. The animals were weighed weekly. The possible differences in weight-gain between the noise-exposed animals and control animals were tested with t-tests. Care and use of the animals reported on in this paper were approved by the Animal Care and Use Committee of the Faculty of Medicine, Utrecht University, under number DEC-GNK-95011.

\section{Protocol and noise exposure}

Rats were assigned at random to the following four groups (eight rats per group): control (exposed to ambient noise of about $65 \mathrm{~dB}$ ) and N90, N100 and N110 exposed to 90,100 and $110 \mathrm{~dB}$ SPL broad-band noise, respectively.

The rats were exposed in wire mesh cages (1: $40 \mathrm{~cm}$; w: $25 \mathrm{~cm}$; h: $15 \mathrm{~cm}$; four rats per cage), with an acoustically transparent mesh floor. The cages were placed in Hazleton cabins (standard whole-body inhalation exposure chambers) with a dimension of approximately $2 \times 2 \times 2$ metre. Noise was generated (precision noise generator type 8075A, Hewlett Packard), amplified (Akai AM-17 or Sony AF470) and presented through four piezo ceramic tweeters (Motorola L054) mounted 0.34 metre above the cages. The resulting sound field was measured, without the rats present, at multiple locations to assess the uniformity of the noise (using a $1 / 4$ " Brüel \& Kjær microphone (type 4135) and a measuring amplifier (Brüel \& Kjær, type 2610) with linear filter settings). Typically, the variation in broad-band noise level from location to location was within $1 \mathrm{~dB}$. One-third-octave-band spectra $(50 \mathrm{~Hz}-40 \mathrm{kHz})$ were obtained with a spectrum analyser (Stanford Research Systems, model SR760 FFT). These one-third-octave-band spectra of the four conditions are depicted in Fig. 1. Ambient noise was below $50 \mathrm{~dB}$ SPL over most of the frequency range. At very low 
frequencies $(500 \mathrm{~Hz})$, it was highly variable and could reach levels up to $60 \mathrm{~dB}$ SPL, which explains why in Fig. 1 the ambient noise level in the $500 \mathrm{~Hz}$ band is higher than that of the $90 \mathrm{~dB}$ exposure level. Most energy in the noise exposure fell between 2 and $12.5 \mathrm{kHz}$.

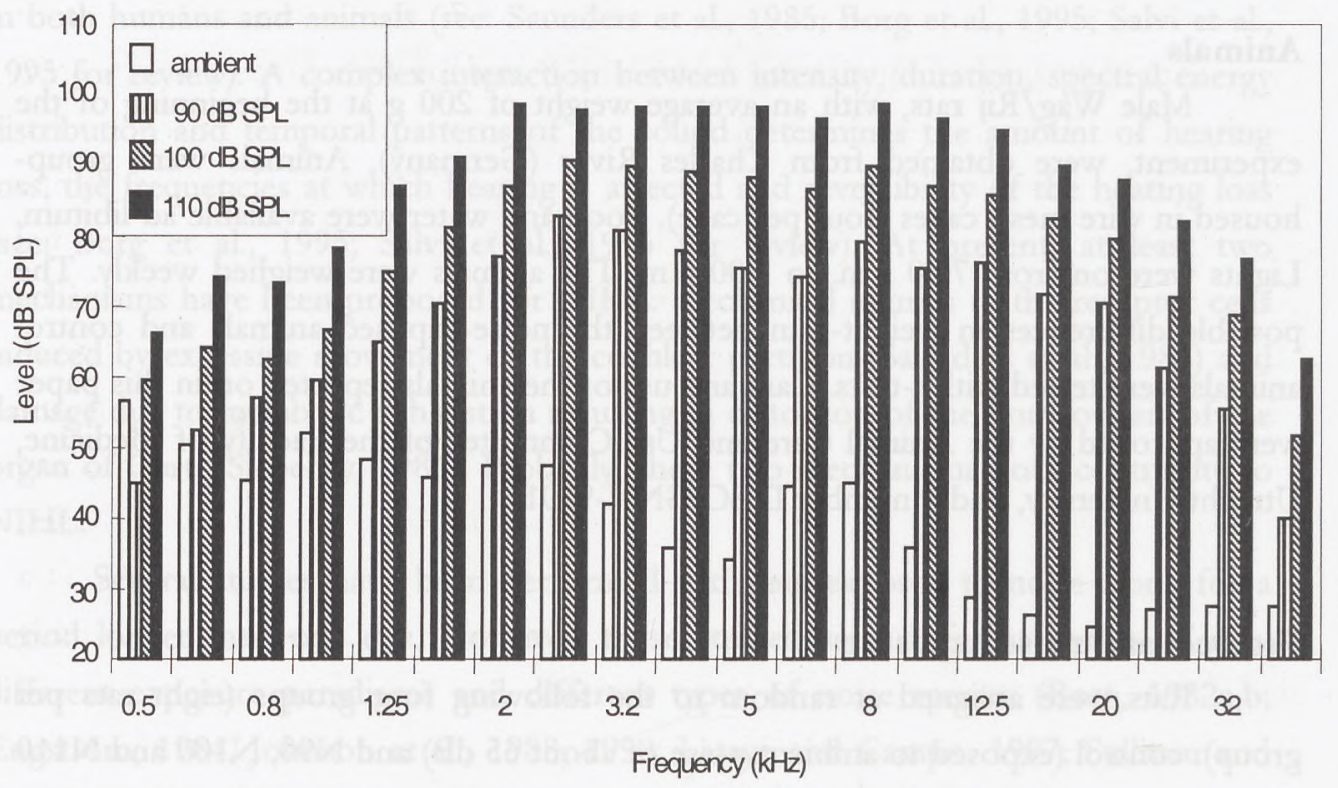

Figure 1. One-third-octave-band noise spectra of the four exposure conditions.

Measurement of $2 \mathrm{f}_{1}-\mathrm{f}_{2}$ distortion product otoacoustic emissions (DPOAEs), electrocochleography and harvesting of the cochleas took place between 3 to 7 weeks after the noise exposure. Generally, two animals were processed per day. Each day, we obtained animals from an experimental group different from that of the day before, to avoid unwanted bias. The 3 to 7 weeks post-exposure interval was chosen because noise effects that still exist 20 to 30 days after the last exposure, are considered to be permanent (Salvi et al., 1995).

The rats were anaesthetised during surgery and during the DPOAE and electrophysiological measurements. The animals were anaesthetised with an intramuscular injection of Thalamonal $(0.1 \mathrm{ml} / 100 \mathrm{~g}$ body weight; Thalamonal contains $2.5 \mathrm{mg} / \mathrm{ml}$ droperidol and $0.05 \mathrm{mg} / \mathrm{ml}$ fentanyl). This was followed by ventilation with a gas mixture containing $33 \% \mathrm{O}_{2}, 66 \% \mathrm{~N}_{2} \mathrm{O}$ and $1 \%$ Halothane through a trachea cannula. Heart frequency was monitored and temperature was kept 
at $38^{\circ} \mathrm{C}$. The rat was placed on its back in an animal holder and the pinnae were resected.

\section{Stimulus generation, distortion product recording and system calibration}

Stimulus generation and acquisition of DPOAEs were controlled by a host computer, with a signal processing board (AP2) and modular audiometry system (Tucker Davis Technology, Gainesville, USA). This system was controlled by custommade software. Two primaries $\left(f_{1}\right.$ and $\left.f_{2}\right), 240 \mathrm{~ms}$ in duration, were generated by the computer and sent to two separate D/A converters. Subsequently, they were fed through low-pass cut-off filters ( $36 \mathrm{kHz})$, attenuators and headphone buffers. The resulting stimuli were delivered to the ear canal through an ER-10C probe system (Etymotic Research, Elk Grove Village, USA), incorporating two speakers and one low-noise microphone. The recorded microphone signal was amplified (40 dB) and A/D converted. Data acquisition was started $100 \mathrm{~ms}$ after the onset of the primaries, to avoid start-up transients. The primaries and the response were both sampled at a rate of $66.67 \mathrm{kHz}$. The recorded signals were time-averaged, windowed (Hanning shaped) and transformed by fast Fourier analysis.

To calibrate the microphone of the probe system in $\mathrm{dB}$ SPL, a microphone correction table was generated, using the frequency-response of the ER-10C microphone, provided by the manufacturer. At the beginning of each experiment, the probe was positioned in the ear canal and the primaries were adjusted per ear. According to the table, $\mathrm{f}_{1}$ and $\mathrm{f}_{2}$ were presented at $60 \mathrm{~dB}$ SPL and the responses were measured via the ER-10C microphone. If the measured amplitudes of the primaries deviated from the target amplitude, the attenuator settings were adjusted.

Amplitude growth curves of the $2 \mathrm{f}_{1}-\mathrm{f}_{2}$ DPOAE were obtained for the primary $f_{2}$ frequencies $4,8,16$ and 22.6 (half an octave above 16$) \mathrm{kHz}$, where the $\mathrm{f}_{2} / \mathrm{f}_{1}$ ratio was 1.25. The levels of the primaries were $L_{2}=L_{1}-10$ and the intensity of $L_{1}$ was increased in $5 \mathrm{~dB}$ steps from 40 to $80 \mathrm{~dB}$ SPL. Each pair of primaries was presented 8 times. DPOAE growth curves were obtained from both ears.

The level of the noise floor at the frequency of the DPOAE was measured with the same procedure and using the same recording and stimulus set up, immediately before the $2 f_{1}-f_{2}$ determination, but with the $f_{1}$ and $f_{2}$ switched off.

Analysis of variance (ANOVA) was performed on the DPOAE growth curves measured after the respective noise exposures, for all tested frequencies and averaged 
over right and left ears. Noise exposure was a between-subjects factor and frequency and level of stimulation were within-subject factors. Post hoc Tukey's HSD test was used to further examine significant differences.

\section{Electrocochleography}

Electrocochleography was performed immediately after the distortion product measurement. Techniques for electrocochleography were described previously (Stengs et al., 1997) and are only summarised here. Auditory evoked responses were recorded differentially with a silverball electrode at the apex of the cochlea and a reference electrode in the muscles of the neck. Trains of $8 \mathrm{~ms}$ tone bursts of $1,2,4,8,12,16$ and $24 \mathrm{kHz}$ were presented, with cosine shaped rise and fall time of $1 \mathrm{~ms}$, except at 1 $\mathrm{kHz}(2 \mathrm{~ms})$ and $2 \mathrm{kHz}(1.5 \mathrm{~ms})$. Consecutive tone bursts were presented with alternating polarity. The inter-burst interval was $99 \mathrm{~ms}$. The responses were preamplified (100 times), band-pass filtered $(-12 \mathrm{~dB} /$ octave, $-3 \mathrm{~dB}$ at $1 \mathrm{~Hz}$ and $10 \mathrm{kHz})$, $\mathrm{A} / \mathrm{D}$ converted at a sample frequency of $33.3 \mathrm{kHz}$ and averaged. The averaged responses to the opposite polarity tone bursts were saved separately for off-line analysis. The compound action potential (CAP) was obtained by addition of the responses of opposite polarity. The CAP amplitude was defined as the difference between the highest negative peak and the summating potential in the complex waveform. The threshold is defined as the stimulus level that produced a $1 \mu \mathrm{V}$ CAP response (iso-response level).

The CAP data were logarithmically transformed before analysis to improve homogeneity of variance and to make proportional effects linear. Subsequently, data from left and right ears were averaged for each stimulus condition. ANOVA was performed as for the DPOAEs.

\section{Histology}

Immediately after electrocochleography, the cochleas of the rats were fixed by transcardial perfusion with a chilled tri-aldehyde fixative consisting of $3 \%$ glutaraldehyde, $2 \%$ formaldehyde, $1 \%$ acrolein, $2.5 \%$ dimethylsulfoxide in $0.1 \mathrm{M}$ sodium cacodylate buffer ( $\mathrm{pH}$ 7.4), followed by overnight immersion in the same fixative at $4^{\circ} \mathrm{C}$. Subsequently, cochleas were processed according to the routine method used for guinea pigs (De Groot et al., 1987). 
Semithin $(1 \mu \mathrm{m})$ midmodiolar sections were cut and stained with $1 \%$ methylene blue, $1 \%$ azur II in 1\% tetraborate. Sections were studied under the light microscope. In a midmodiolar section of the rat cochlea, the organ of Corti was examined at five locations, separated by a half-turn distance. Hair cell counts were performed at each of these five locations in both the left and right cochlea, using 5-6 serial sections. If a hair cell is not seen in these 5-6 serial $1 \mu \mathrm{m}$ sections, it can be reliably assumed that the cell is not present. Two investigators, independently of one other, in a single-blind fashion performed all OHC counts. Estimates were made of the characteristic frequencies of the locations along the basilar membrane at which the $\mathrm{OHCs}$ were counted; the corresponding frequencies were calculated to be $52,21,11,4$ and $0.1 \mathrm{kHz}$, according to a frequency map published by Müller (1991).

The results for the left and right cochleas were pooled and averaged per location. ANOVA was used for statistical evaluation of the $\mathrm{OHC}$ counts. Noise exposure was a between-subjects factor, cochlear location a within-subjects factor.

\section{RESUlts}

\section{General health}

During the experiment, the rats exhibited no overt signs of ill health following the noise exposure. At the end of the treatment period the weight of the exposed rats was not different from that of the control animals ( $\mathrm{t}$-test, $\mathrm{P}=0.39$ ).

\section{Distortion product otoacoustic emission}

DPOAE growth curves for $2 \mathrm{f}_{1}-\mathrm{f}_{2}$ amplitude of the four groups are presented in Fig. 2, for all tested frequencies and averaged for left and right ears per stimulus condition. Obvious deterioration of the DPOAE growth curves for the N110 group was observed at 4, 8 and $16 \mathrm{kHz}$. At $22.6 \mathrm{kHz}$, the decrease was less severe. After 90 and $100 \mathrm{~dB}$ SPL noise, the DPOAE growth curves resembled that of the control group. However, a trend of enhancement of the growth curves for the N90 and N100 group could be distinguished at $22.6 \mathrm{kHz}$ for the highest primary levels.

ANOVA performed on the whole data set of DPOAE growth curves (all frequencies and stimulus levels included) showed main effects of noise level, frequency and stimulus level and significant interactions. The significant interaction 
between noise level and frequency was further pursued by ANOVA performed at each frequency separately. A significant effect of noise level was found at $4 \mathrm{kHz}$ $\left(\mathrm{F}_{(3,27)}=4.9 ; \mathrm{P}<0.01\right), 8 \mathrm{kHz}\left(\mathrm{F}_{(3,27)}=49.3 ; \mathrm{P}<0.01\right)$ and $16 \mathrm{kHz}\left(\mathrm{F}_{(3,27)}=8.8 ; \mathrm{P}<0.01\right)$. No significant decrease was found at $22.6 \mathrm{kHz}$ after the $110 \mathrm{~dB}$ SPL noise, although Fig. 2 suggested such a trend. Subsequently, post hoc Tukey HSD tests were performed at each frequency. They showed differences in DPOAE growth curves between the control group and that of the $\mathrm{N} 110$ group at 4,8 and $16 \mathrm{kHz}$ (all $\mathrm{P}<0.01$ ).
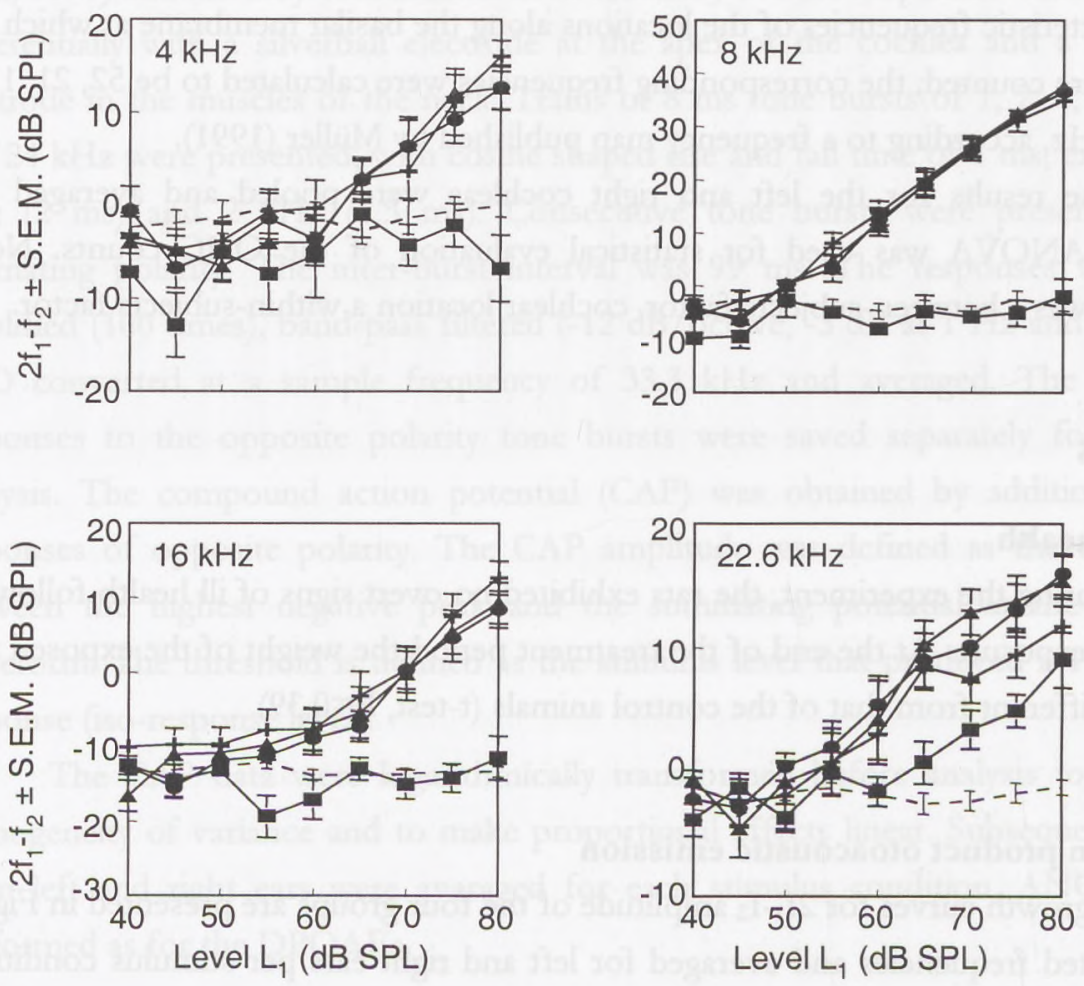

Figure 2. The effects of noise exposure on the DPOAE $\left(2 f_{1}-f_{2}\right)$ growth curves (mean \pm S.E.M.), displayed as function of the stimulus level of $f_{1}\left(L_{1}\right):+=$ control group, $\boldsymbol{\Delta}=90$ dB SPL, $\bullet=100 \mathrm{~dB} S P L, \mathbf{\square}=110 \mathrm{~dB} S P L$, dashed line = background noise .

\section{Electrocochleography}

CAP amplitude growth curves are shown in Fig. 3, for all tested frequencies and averaged over left and right ears. An apparent effect of noise exposure level was observed in the N110 group at 4, 8, 12, 16 and $24 \mathrm{kHz} .100 \mathrm{~dB}$ SPL noise only slightly 

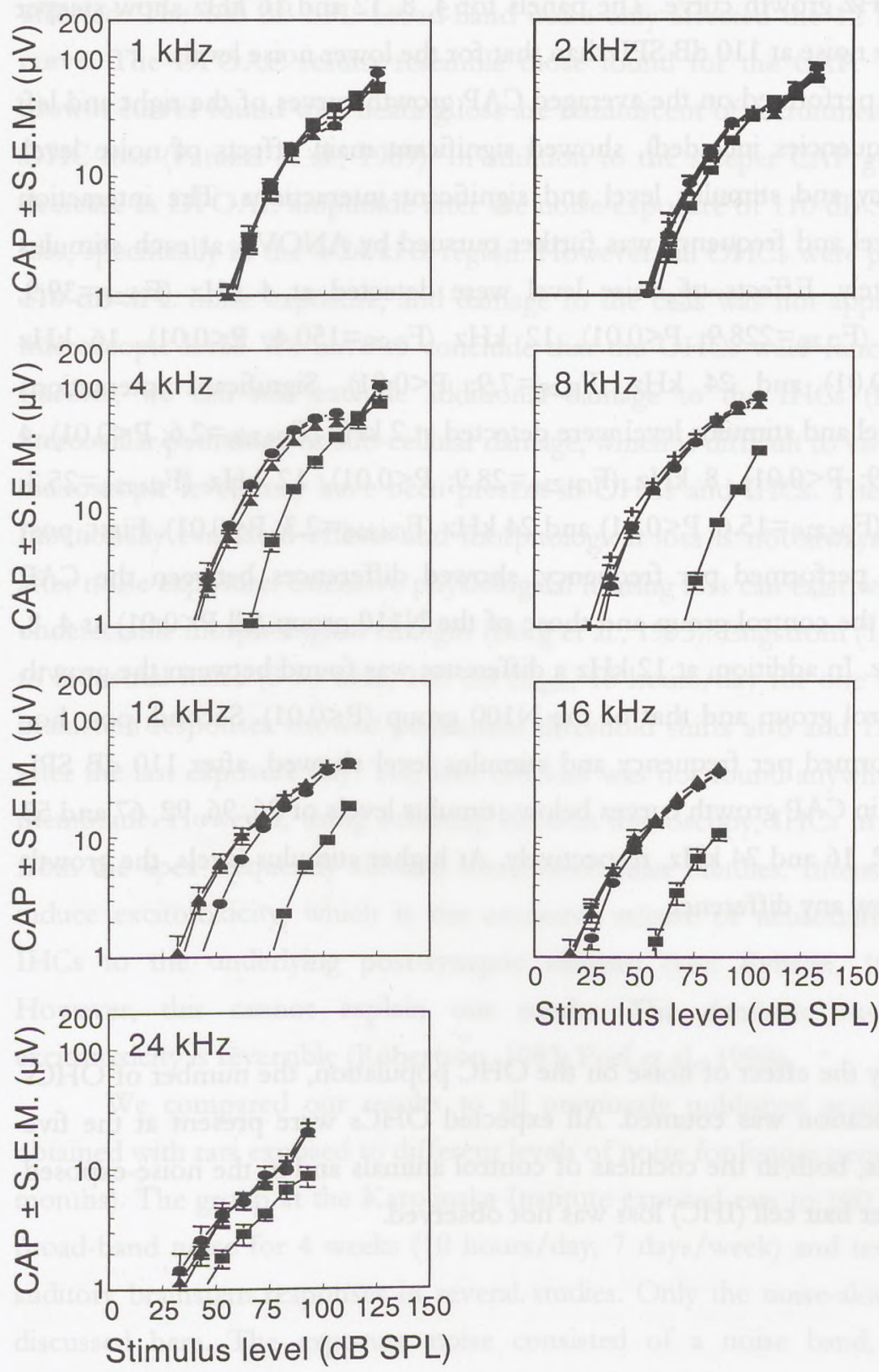

Stimulus level (dB SPL)

Figure 3. Effects of noise exposure on the CAP amplitude growth curves (mean \pm S.E.M.) at: $1,2,4,8,12,16$ and $24 \mathrm{kHz}:+=$ control group, $\boldsymbol{\Delta}=90 \mathrm{~dB} \mathrm{SPL}, \bullet=100 \mathrm{~dB} \mathrm{SPL}$, - $=110 \mathrm{~dB} S P L$. 
affected the $12 \mathrm{kHz}$ growth curve. The panels for 4, 8, 12 and $16 \mathrm{kHz}$ show steeper growth curves for noise at $110 \mathrm{~dB}$ SPL than that for the lower noise levels.

ANOVA, performed on the averaged CAP growth curves of the right and left ears (with all frequencies included), showed significant main effects of noise level, stimulus frequency and stimulus level and significant interactions. The interaction between noise level and frequency was further pursued by ANOVA at each stimulus frequency separately. Effects of noise level were detected at $4 \mathrm{kHz} \quad\left(\mathrm{F}_{(3,28)}=39.9\right.$; $\mathrm{P}<0.01), 8 \mathrm{kHz}\left(\mathrm{F}_{(3,28)}=228.9 ; \mathrm{P}<0.01\right), 12 \mathrm{kHz}\left(\mathrm{F}_{(3,28)}=150.4 ; \mathrm{P}<0.01\right), 16 \mathrm{kHz}$ $\left(\mathrm{F}_{(3,28)}=96.2 ; \mathrm{P}<0.01\right)$ and $24 \mathrm{kHz}\left(\mathrm{F}_{(3,28)}=7.9 ; \mathrm{P}<0.01\right)$. Significant interactions between noise level and stimulus level were detected at $2 \mathrm{kHz}\left(\mathrm{F}_{(24,224)}=2.6 ; \mathrm{P}<0.01\right), 4$ $\mathrm{kHz}\left(\mathrm{F}_{(24,224)}=43.9 ; \mathrm{P}<0.01\right), 8 \mathrm{kHz} \quad\left(\mathrm{F}_{(24,224)}=28.9 ; \mathrm{P}<0.01\right), 12 \mathrm{kHz}\left(\mathrm{F}_{(24,224)}=25.3\right.$; $\mathrm{P}<0.01), 16 \mathrm{kHz}\left(\mathrm{F}_{(24,224)}=15.6 ; \mathrm{P}<0.01\right)$ and $24 \mathrm{kHz}\left(\mathrm{F}_{(24,224)}=2.3 ; \mathrm{P}<0.01\right)$. First, post hoc Tukey HSD performed per frequency, showed differences between the CAP growth curves of the control group and those of the N110 group (all $\mathrm{P}<0.01$ ) at 4,8 , 12,16 and $24 \mathrm{kHz}$. In addition, at $12 \mathrm{kHz}$ a difference was found between the growth curve of the control group and that of the $\mathrm{N} 100$ group $(\mathrm{P}<0.01)$. Second, post hoc Tukey HSD performed per frequency and stimulus level showed, after $110 \mathrm{~dB}$ SPL noise, differences in CAP growth curves below stimulus levels of 96, 96, 92, 67 and 53 dB SPL at 4, 8, 12, 16 and $24 \mathrm{kHz}$, respectively. At higher stimulus levels, the growth curves did not show any difference.

\section{Histology}

To quantify the effect of noise on the $\mathrm{OHC}$ population, the number of $\mathrm{OHCs}$ present at each location was counted. All expected OHCs were present at the five examined locations, both in the cochleas of control animals and in the noise-exposed animals. Also, inner hair cell (IHC) loss was not observed.

\section{DISCUSSION}

Three groups of eight rats were exposed to broad-band noise at levels of 90 , 100 and $110 \mathrm{~dB}$ SPL for 8 hours/day and 5 consecutive days. An additional group of eight rats (exposed to the ambient noise at $65 \mathrm{~dB}$ SPL) served as the control group. Effects on hearing were abundant after the highest noise level: the DPOAE growth curves at 4,8 and $16 \mathrm{kHz}$ and the $\mathrm{CAP}$ growth curves at 4, 8, 12, 16 and $24 \mathrm{kHz}$ were 
affected. The $100 \mathrm{~dB}$ SPL broad-band noise only affected the $12 \mathrm{kHz}$ CAP growth curve. The DPOAE results resemble those found for the CAP. The steeper CAP growth curves found with hearing loss are reminiscent of recruitment, which indicates OHC loss (Patuzzi et al., 1989). In addition to the steeper CAP growth curves, the decrease in DPOAE amplitude after the noise-exposure of $110 \mathrm{~dB}$ SPL suggest OHC loss, specifically in the $4-24 \mathrm{kHz}$ region. However, all $\mathrm{OHCs}$ were present, even after $110 \mathrm{~dB}$ SPL noise exposure, and damage to the cells was not apparent at the lightmicroscopic level. We have to conclude that the OHCs were functionally damaged. Further, we can not exclude additional damage to the IHCs (Engström, 1984). Stereociliar pathology or sub-cellular damage, which is difficult to visualise at the lightmicroscopic level, may have been present in OHCs and IHCs. The relation between functionally evaluated effects and morphological loss is not always straight forward after noise exposure: extensive physiological hearing loss can exist with minor or even undetectable morphological changes (Borg et al., 1995). Engström (1984) exposed rats to industrial noise ( $3-30 \mathrm{kHz}, 100 \mathrm{~dB} \mathrm{~L}_{\text {eq, lin, }} 10$ hours/day for one month). Auditory brainstem responses showed permanent threshold shifts at 6 and $12 \mathrm{kHz}$ two weeks after the last exposure day. Yet, hair cell loss was not found anywhere on the basilar membrane. However, using scanning electron microscopy, IHCs in the area 6-8 $\mathrm{mm}$ from the apex frequently showed fused stereociliar bundles. Intense sound can also induce excitotoxicity, which is the excessive release of neurotransmitter from the IHCs to the underlying post-synaptic element (see: Eybalin, 1993 for review). However, this cannot explain our results. The dendritic swelling caused by excitotoxicity is reversible (Robertson, 1983; Puel et al., 1998).

We compared our results to all previously published results known to us, obtained with rats exposed to different levels of noise for longer periods (1 week to 15 months). The group at the Karolinska Institute exposed rats to $100 \mathrm{~dB} \mathrm{~L}_{\text {eq, lin }}$ variable broad-band noise for 4 weeks ( 10 hours/day, 7 days/week) and tested hearing with auditory brainstem responses in several studies. Only the noise-alone results will be discussed here. The exposure noise consisted of a noise band, $1680 \mathrm{~Hz}$ wide, sweeping from 3 to $30 \mathrm{kHz}$. Most of the energy fell between 5 and $15 \mathrm{kHz}$ (Borg, 1982a; Engström, 1984; Johnson et al., 1988, 1990). Maximum hearing loss was $50 \mathrm{~dB}$ at $12.5 \mathrm{kHz}$ (Johnson et al., 1988), $26 \mathrm{~dB}$ at $12.5 \mathrm{kHz}$ (Johnson et al., 1990) and $30 \mathrm{~dB}$ between 12 and $20 \mathrm{kHz}$ (Borg, 1982a). Borg (1982b) exposed rats for periods of up to 15 months with the noise protocol described above. Hearing loss kept increasing with 
exposure duration and reached a maximal of $75 \mathrm{~dB}$ at 15 months $(37 \mathrm{~dB}$ at 1 month, $44 \mathrm{~dB}$ at 2 months and $62 \mathrm{~dB}$ at 3 months; maximal loss always between 20 and 44 $\mathrm{kHz}$ ). Sullivan and Conolly (1988) exposed rats to $110 \mathrm{~dB}$ broad-band noise with peak energy at 0.5 and $20 \mathrm{kHz}$ respectively for 6 hours/day, 5 days/week for 4 weeks. Maximum hearing loss was found around $50 \mathrm{~dB}$ between 4 and $8 \mathrm{kHz}$. Lataye and Campo (1997) exposed rats for 6 hours/day, 5 days/week for 4 weeks to a $92 \mathrm{~dB}$ octave band noise centred at $8 \mathrm{kHz}$. Auditory function was tested by recording brainstem auditory evoked potentials. A $30 \mathrm{~dB}$ hearing loss was detected after the exposure at 10-12 kHz. Rats exposed to $95 \mathrm{~dB}$ SPL broad-band noise (18 hours/day, 5 days/week, 3 weeks; energy peak between 2 and $16 \mathrm{kHz}$ ) showed a maximum threshold shift of $25 \mathrm{~dB}$ at $8 \mathrm{kHz}$ with reflex modification audiometry (Muijser et al., 2000). The maximum loss in this study was $42 \mathrm{~dB}$ at $12 \mathrm{kHz}$, about half an octave above the peak in the energy spectrum of the $110 \mathrm{~dB}$ SPL noise.

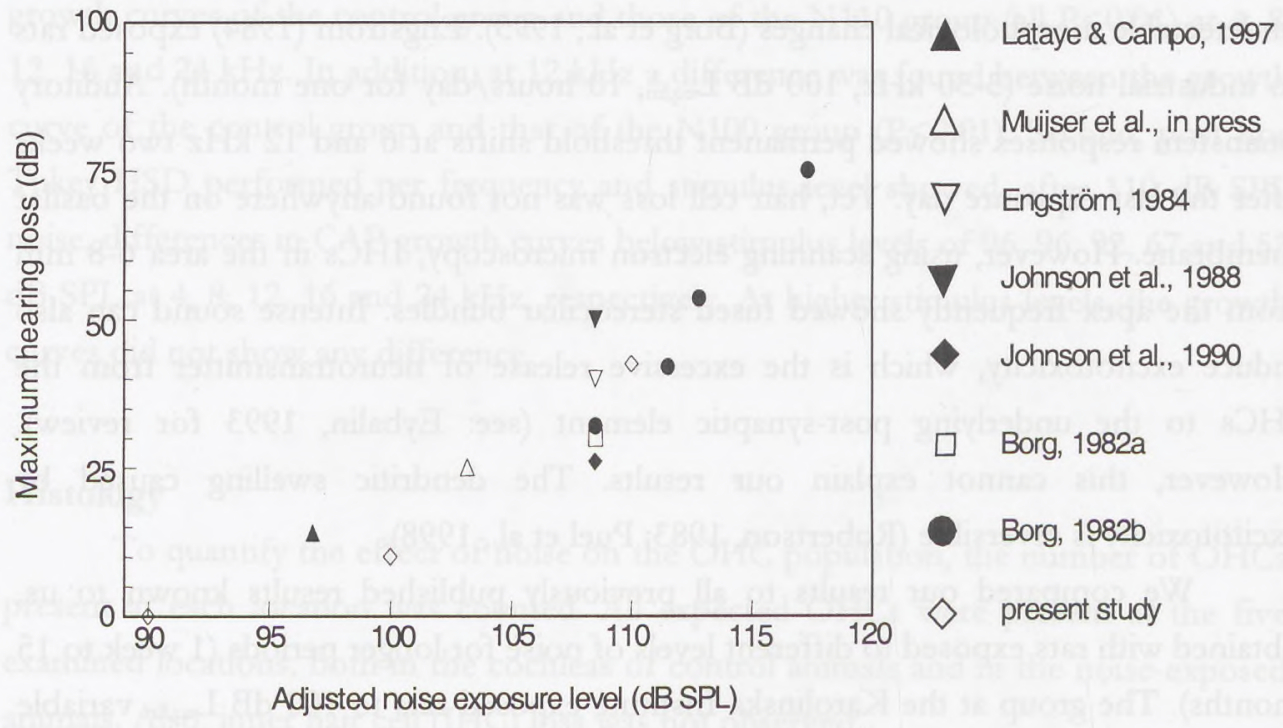

Figure 4. Comparison of noise-induced threshold shifts found in literature and in the present study. The ordinate gives the maximal threshold shift; The abscissa the noise exposure level adjusted to a one week, 5 days, 8 hours per day equivalent level.

As a common measure for noise exposition in the various studies, we calculated the total exposure based upon the equal-energy theory. The equal-energy theory proposes that the damaging effect of noise on hearing depends directly upon the total sound energy in such a way that there is a trade-off between the level of the 
exposure and its duration (Vertes et al., 1981; Ward et al., 1981). This principle was formulated to estimate hearing loss in workers who are exposed for many years. We used the following formula to adjust the noise exposures in the various rat studies to an equivalent 8 hours/day, 5 days/week, 1 week exposure (the exposure of our study):

$$
10 \bullet{ }^{10} \log \{(\mathrm{h} / 8) \bullet(\mathrm{d} / 5) \bullet \mathrm{w}\}
$$

in which $\mathrm{h}=$ hours per day, $\mathrm{d}=$ days per week and $\mathrm{w}=$ number of weeks of exposure

Fig. 4 shows the result of this comparison. On the horizontal axis the adjusted exposure levels are presented. On the vertical axis we plotted the maximum hearing loss at any frequency encountered in the various studies, independently of the frequency spectrum of noise or what method was used to evaluate the hearing loss (behavioural, brainstem evoked potentials or electrocochleography). All hearing loss was expressed in threshold shift $(\mathrm{dB})$. This comparison shows a consistent relation amongst the results from the studies included. The higher the adjusted noise exposure level, the higher the accompanying hearing loss. This relationship is clear, despite the fact that the types of noise, exposure regimens used and method of evaluating hearing loss were variable in the studies included in this comparison. Thus, the equal-energy concept provides a useful descriptor of hearing loss in rats exposed to noise for 6 to 18 hours per day, 5 to 7 days per week over periods of 1 week to 15 months at equivalent levels of 95 to $120 \mathrm{~dB}$ re an 8 hour, 5 days a week, one week exposure. Fig. 4 demonstrates that the results of the present study are well in line with the previous results.

In conclusion, this study (and previous studies) show that it takes 100 to 110 $\mathrm{dB}$ SPL noise to cause measurable damage to the rat cochlea in an exposure paradigm representative of industrial situations. This information will be valuable for ongoing experiments into the combined effect of exposure to noise and ototoxic chemicals.

\section{ACKNOWLEDGMENTS}

This study was supported by grants of NWO-MW (grant-number 9004-63085) and by the Heinsius-Houbolt Foundation. The authors would like to thank J.C.M.J. de Groot, R. Bulder and F. Hendriksen for their histological assistance. 


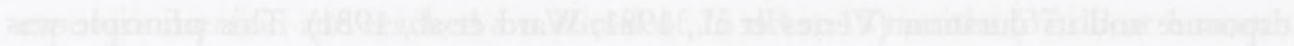

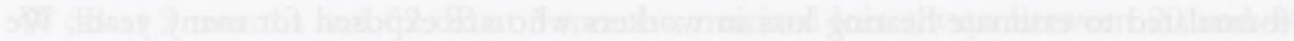

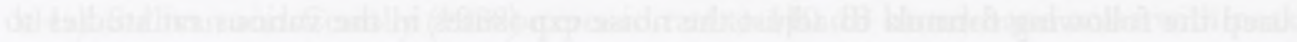

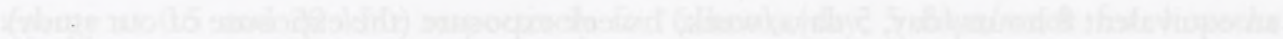

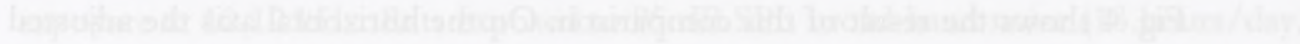

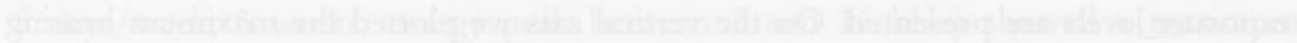

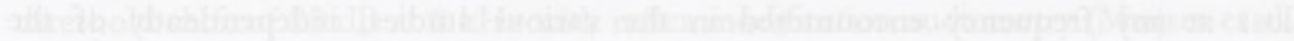

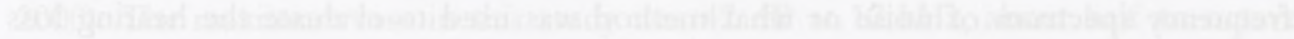

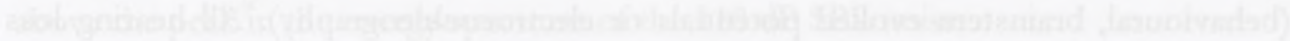

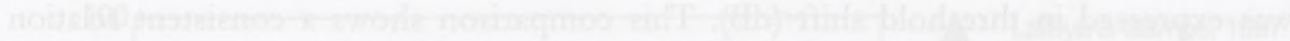

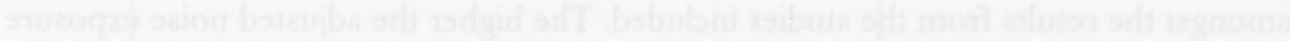

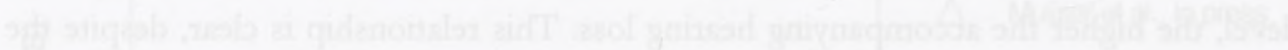

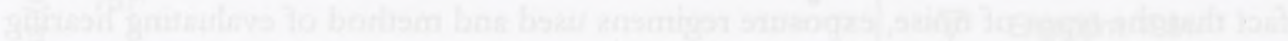

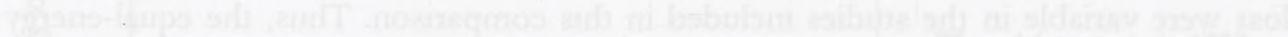

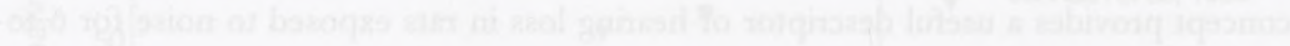

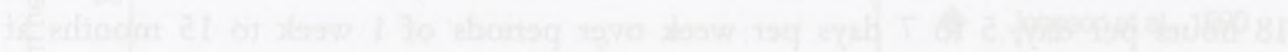

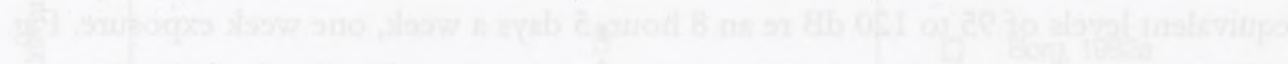

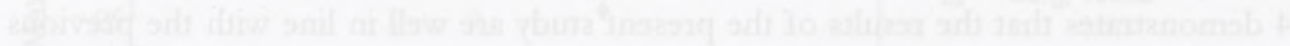

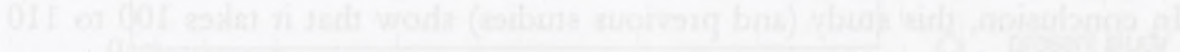

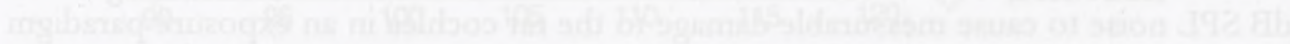

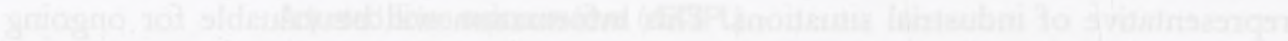

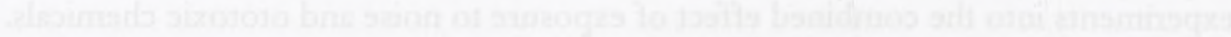




\section{EFFECTS OF SIMULTANEOUS EXPOSURE TO ETHYL BENZENE AND BROAD-BAND NOISE ON HEARING IN RATS}

The effects on hearing of simultaneous exposure to the ototoxic organic solvent ethyl benzene and broad-band noise were evaluated in rats. The effects of three ethyl benzene concentrations ( $0 \mathrm{ppm}, 300 \mathrm{ppm}$ or $400 \mathrm{ppm}$ ) and three noise levels (95 or $105 \mathrm{dBlin}$ SPL or background noise at $65 \mathrm{~dB}_{\operatorname{lin}} \mathrm{SPL}$ ) and all their combinations were investigated. The animals were exposed for eight hours/day for 5 days. Distortion product otoacoustic emissions (DPOAEs) and compound action potentials (CAPs) were affected after $105 \mathrm{~dB}$ noise alone, and after $105 \mathrm{~dB}$ noise in combination with ethyl benzene (300 ppm or 400 $\mathrm{ppm})$. However, the amount of loss after exposure to these combinations did not exceed the loss induced after $105 \mathrm{~dB}$ noise alone. All other exposures did not affect DPOAEs or CAPs significantly. Outer hair cell (OHC) loss after exposure to $300 \mathrm{ppm}$ ethyl benzene was located in the third row of OHCs. When the concentration was increased, the loss spread out to the second and first row of OHCs. Noise hardly affected the OHC counts except for minor loss in the first row of OHCs after $105 \mathrm{~dB}$ SPL. Synergistic interactions were not significant in the physiological measurements, but they were clearly present in OHC counts.

(Submitted; co-authors: Klis, Muijser, Kulig, Smoorenburg) 


\section{INTRODUCTION}

Simultaneous exposure to noise and chemicals is not uncommon in workplaces. Exposure to noise may result in damage to the inner ear. Noise-induced hearing loss (NIHL) is a significant occupational health problem in industrialized countries (Alberti, 1998). Besides noise, a wide range of chemicals can impair inner ear function. Among these ototoxic agents are the organic solvents, which are used as degreasers, adhesives, fuel additives, glues and thinners. Human and animal studies have shown that several organic solvents can induce hearing loss in the mid-frequency region of the cochlea (e.g., Morata and Dunn, 1994; Crofton et al., 1994).

A point of concern with ototoxic agents is that they may interact synergistically when administered together with noise (Rybak, 1992). In the present paper, synergism is defined as an interaction, which leads to a combined effect that is larger than the arithmetic sum of the effects observed with each exposure separately. Barregard and Axelsson (1984) were among the first to suggest a synergistic interaction between noise and solvents. They tested the hearing of shipyard painters who were exposed to noise as well as to organic solvents. They reported a more pronounced hearing loss, based on tone audiograms, than the hearing loss that would be predicted from noise exposure alone. Possible interactions between noise and organic solvents were further investigated by a number of authors. Despite these investigations, a controversy still exists: some studies provided evidence that there is a synergistic interaction between noise and solvent exposure in humans (Morata et al., 1993; Morata and Dunn, 1994), while others did not find any synergistic effect (Jacobsen et al., 1993; Sass-Kortsak et al., 1995).

In addition to the epidemiological results described above, animal studies have been performed to investigate the combined effects of noise and organic solvents on hearing. An interaction between noise and toluene was reported by Johnson et al. (1988). Rats were first exposed to $1000 \mathrm{ppm}$ toluene (16 hours/day, 5 days/week, 2 weeks). This was followed by $100 \mathrm{~dB} \mathrm{~L}_{\text {eq }}$ noise (10 hours/day, 7 days/week, 4 weeks). The hearing loss was determined by evaluating brainstem auditory evoked potentials. The combination of toluene and noise resulted in a synergistic interaction (Johnson et al., 1988). However, when the sequence was reversed, with noise exposure preceding toluene exposure, the hearing loss in $\mathrm{dB}$ did not exceed the summated loss caused by noise alone and toluene alone (Johnson et al., 1990). In a more recent experiment by 
Lataye and Campo (1997), rats were exposed simultaneously to toluene (2000 ppm) and noise (92 dB SPL) for 6 hours/day, 5 days/week, 4 weeks. The auditory function was tested by recording brainstem auditory evoked potentials. This study confirmed the synergistic interaction between toluene and noise in rats. Muijser et al. (2000) worked with another ototoxic organic solvent, trichloroethylene. Rats simultaneously exposed to 3000 ppm of this compound and $95 \mathrm{~dB}$ SPL broad band noise (18 hours/day, 5 days/week for 3 weeks) showed a significantly enlarged threshold shift at $4 \mathrm{kHz}$, measured with reflex modification audiometry, as compared to the sum of the threshold shifts in $\mathrm{dB}$ induced after exposure to noise alone and trichloroethylene alone. However, at other frequencies tested $(8,16$ and $20 \mathrm{kHz})$ this synergistic effect did not appear. Finally, the effects of combined exposure to $750 \mathrm{ppm}$ styrene and a 97 $\mathrm{dB}$ SPL octave band of noise centred at $8 \mathrm{kHz}$ ( 6 hours/day, 5 days/week for 4 weeks) on hearing were examined in the rat (Lataye et al., 2000). The combination induced synergistic loss in the amplitudes of brainstem auditory evoked potentials. Furthermore, OHC loss exceeded the summated loss due to exposure to one of the agents alone.

The present study was designed to evaluate whether an additive or synergistic interaction will occur after simultaneous exposure to the organic solvent ethyl benzene and noise. This solvent is probably the ototoxic solvent most widely used (American Chemical Society, 1996). Moreover, Pryor and Rebert (1993) reported that ethyl benzene was one of the most potent ototoxic organic solvents tested in their comparative study. In spite of these facts, the ototoxicity of ethyl benzene has not been extensively examined. Studies in our laboratory confirmed the ototoxic potency of ethyl benzene (Cappaert et al., 1999; Chapters 2 and 3). In the present study, rats were exposed to ethyl benzene, noise or their combination for 8 hours/day for 5 consecutive days. The ethyl benzene concentrations and noise levels applied in this study were relatively low and meant to cause only minor effects. This choice was made to study the question of whether or not the two toxic agents, considered to be safe individually, may be unsafe in combination. We used a variety of methods to assess hearing loss which included the measurement of distortion product otoacoustic emissions (DPOAEs), electrocochleographic recording of compound action potentials (CAPs) and histopathological analysis involving hair cell counts. 


\section{MATERIALS AND METHODS}

\section{Animals and experimental design}

Wag/Rij rats (obtained from Charles River, Germany) were used. They had an average weight of $200 \mathrm{~g}$ at the beginning of the experiment. The rats were grouphoused in wire-mesh cages (four per cage). Food and water were available ad libitum. Lights were on from 7:00 a.m. to 7:00 p.m. The animals' weight was recorded regularly during the experiment. Possible differences in weight gain between the exposed animals and the control animals was tested with t-tests. Care and use of the animals reported on in this paper were approved by the Animal Care and Use Committee of the University Medical Center Utrecht, under number DEC-GNK-95011.

A factorial design was used to investigate the effects of three levels of noise (95 or $105 \mathrm{~dB}_{\text {lin }} \mathrm{SPL}$ or background noise at $65 \mathrm{~dB}_{\text {lin }} \mathrm{SPL}$ ) and three concentrations of ethyl benzene $(0,300$ or $400 \mathrm{ppm})$ and all combinations. The rats were exposed for 8 hours/day for 5 consecutive days. In case of combined exposure, noise and ethyl benzene were applied simultaneously. The concentrations of ethyl benzene and levels of noise applied in this study were based on dose-response data previously collected in our laboratory (Chapters 3 and 4 in this thesis). 300 ppm ethyl benzene and $95 \mathrm{~dB}$ SPL noise were chosen because there were no effects at these levels in previous studies. The highest noise level and the highest ethyl benzene concentration in the present study induced moderate hearing loss in the $8-12 \mathrm{kHz}$ region in the previous studies. For logistic reasons, the rats were tested in three cohorts of three or four exposure groups (Table 1). Each group consisted of 8 animals. A control group was included in each cohort.

Table 1. The arrangement of the experimental groups into the three subsequent cohorts, indicated with 1, 2 and 3.

\begin{tabular}{lccc}
\hline & $\begin{array}{c}0 \text { ppm ethyl } \\
\text { benzene }\end{array}$ & $\begin{array}{c}300 \text { ppm ethyl } \\
\text { benzene }\end{array}$ & $\begin{array}{c}400 \text { ppm ethyl } \\
\text { benzene }\end{array}$ \\
\hline background noise & $1,2,3$ & 3 & 1 \\
$95 \mathrm{~dB}$ SPL & 1 & 2 & 3 \\
$105 \mathrm{~dB} \mathrm{SPL}$ & 3 & 1 & 2 \\
\hline
\end{tabular}


Measurement of DPOAEs, electrocochleography and harvesting of the cochlea's, all performed on both sides, took place between 3 to 7 weeks after the exposure. This post-exposure interval was chosen because noise-induced hearing loss that still exists 20 to 30 days after the last exposure, is commonly considered to be permanent (Salvi et al., 1995). Generally, two animals were processed per day. Each day, we processed animals from exposure groups different from those of the day before. The rats were anaesthetised during surgery and during the physiological measurements. The animals were given an intramuscular injection of Thalamonal (0.1 $\mathrm{ml} / 100 \mathrm{~g}$ body weight; Thalamonal contains $2.5 \mathrm{mg} / \mathrm{ml}$ Droperidol and $0.05 \mathrm{mg} / \mathrm{ml}$ Fentanyl) followed by artificial ventilation with a gas mixture containing $33 \% \mathrm{O}_{2}, 66 \%$ $\mathrm{N}_{2} \mathrm{O}$ and $1 \%$ Halothane through a trachea cannula. Heart frequency was monitored and temperature was kept at $38^{\circ} \mathrm{C}$. The rat was placed on its back in an animal holder and the pinnae were resected.

\section{Exposures}

The animals were placed in acoustically transparent wire mesh cages $(1: 40 \mathrm{~cm}$; w: $25 \mathrm{~cm}$; h: $15 \mathrm{~cm}$; four rats per cage). The cages were placed in a stainless steel exposure chamber (Hazleton Systems Inc., Aberdeen, UK) with dimensions of approximately $2 \times 2 \times 2 \mathrm{~m}$. The exposure started at 8:30 a.m.

\section{Ethyl benzene}

Ethyl benzene (Merck, Darmstadt, Germany, 99\% pure) was evaporated at an elevated temperature and the saturated gas was injected into the air supply system, which operated at $25 \mathrm{~m} 3 / \mathrm{hr}$. Total carbon analysers (Ratfisch, RS 55-T, Poing, Germany) were used to monitor the ethyl benzene concentration during the exposure. The concentration was kept within $5 \%$ of the desired concentration.

\section{Noise}

Broad-band noise was generated (precision noise generator type 8075A, Hewlett Packard), amplified (Akai AM-17 or Sony AF470) and presented through four piezo ceramic tweeters (Motorola L054) mounted $34 \mathrm{~cm}$ above the cages. The resulting sound field was measured at multiple locations, without the rats present, to check uniformity of the sound level (using a 1/4" Brüel \& Kjær microphone (type 4135) and a measuring amplifier (Brüel \& Kjær, type 2610) with linear filter settings). 
Typically, the variation in broad-band noise level from location to location was within $1 \mathrm{~dB}$. One-third-octave-band spectra $(50 \mathrm{~Hz}-40 \mathrm{kHz})$ were obtained with a spectrum analyser (Stanford Research Systems, Sunnyvale, CA, model SR760 FFT). These onethird-octave-band spectra of the three conditions (background noise and the two experimental noise levels) are depicted in Fig. 1. Ambient noise was below $50 \mathrm{~dB}$ SPL over most of the frequency range. At very low frequencies $(<500 \mathrm{~Hz})$, it was highly variable and could reach levels up to $60 \mathrm{~dB}$ SPL (Fig. 1) and was probably due to the air supply system.

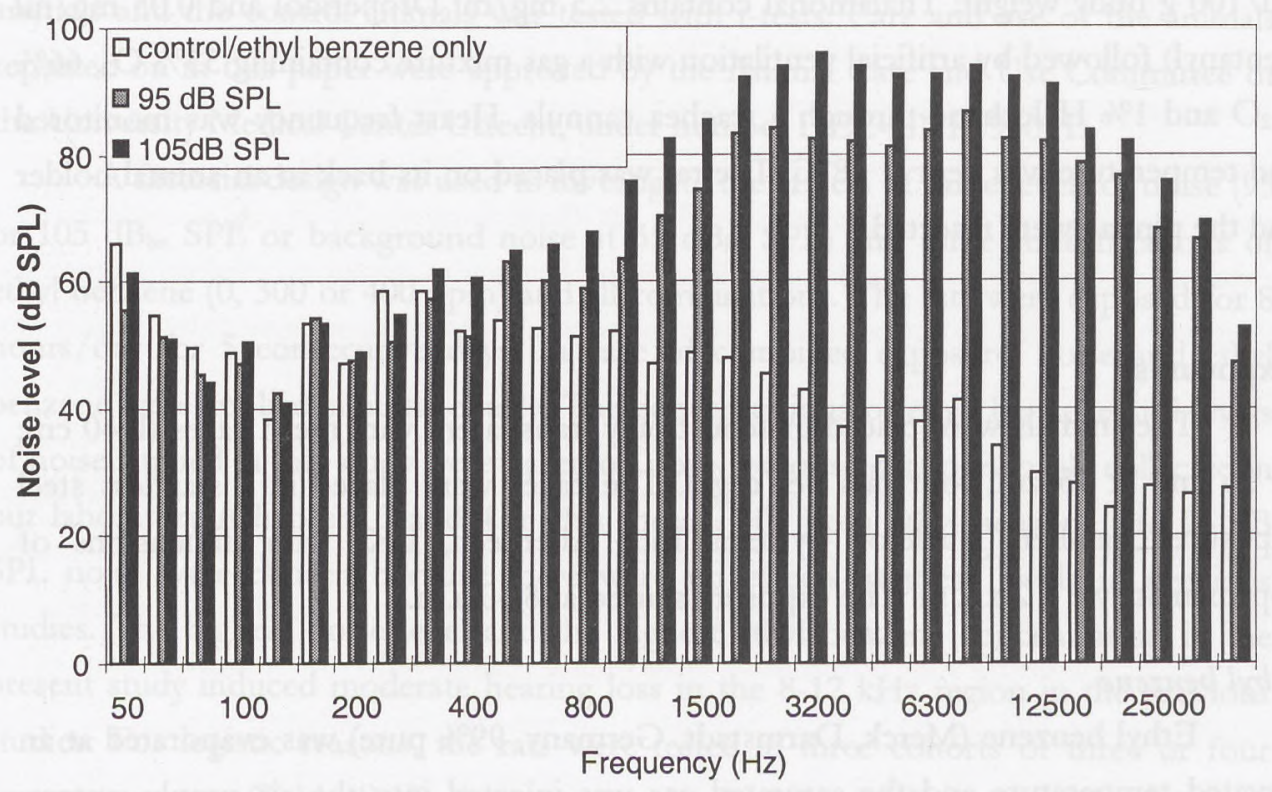

Figure 1. One-third-octave-band noise spectra of the three noise exposure conditions. Most energy is concentrated in the frequency range from 1.5 to $12.5 \mathrm{kHz}$. The high levels of background noise in the low-frequency range are probably due to the air supply system. Total SPL is based on linear weighting.

This explains the high ambient noise levels in the bands below $500 \mathrm{~Hz}$ compared to those of the 95 and $105 \mathrm{~dB}$ SPL exposure level. Most energy in the noise exposure fell between 1.5 and $12.5 \mathrm{kHz}$ which is related to the type of amplifier and tweeter used. The overall background noise level did not exceed $65 \mathrm{~dB}$ SPL in case of control groups and ethyl benzene alone exposure. As explained before, the background noise had most of its energy in frequency bands below $500 \mathrm{~Hz}$, where rats are very insensitive (Fay, 1988). 


\section{Measurements}

\section{Distortion product otoacoustic emissions}

A host computer, with a signal processing board (AP2) and modular audiometry system (Tucker Davis Technologies, Gainesville, FL), controlled stimulus generation and acquisition of the emissions. This system was controlled by custommade software. Two primaries $\left(f_{1}\right.$ and $f_{2}$ ), both $240 \mathrm{~ms}$ in duration, were generated by the computer and sent to two separate D/A converters. The primaries were generated at a sample rate of $66.7 \mathrm{kHz}$. Subsequently, the primaries were fed through low-pass cut-off filters $(36 \mathrm{kHz})$, attenuators and headphone buffers. The resulting stimuli were delivered to the ear canal through an ER-10C probe system (Etymotic Research, Elk Grove Village, IL), incorporating two speakers and one low-noise microphone. The recorded microphone signal was amplified $(40 \mathrm{~dB})$ and $\mathrm{A} / \mathrm{D}$ converted. Data acquisition started $100 \mathrm{~ms}$ after the onset of the primaries, to avoid start-up transients. The emission was sampled at a rate of $66.7 \mathrm{kHz}$. The recorded signals were timeaveraged, windowed (Hanning-shaped) and transformed by fast Fourier analysis. To calibrate the microphone of the probe system in $\mathrm{dB}$ SPL, a microphone correction table was generated, using the frequency response of the ER-10C microphone provided by the manufacturer. At the beginning of each measurement, the probe was positioned in the ear canal and the primaries were adjusted per ear. According to the table, $\mathrm{f}_{1}$ and $\mathrm{f}_{2}$ were presented at $60 \mathrm{~dB}$ SPL and the responses were measured via the ER-10C microphone. If the measured amplitudes deviated from the target amplitude, the attenuator settings were adjusted. Amplitude growth functions of the $2 \mathrm{f}_{1}-\mathrm{f}_{2}$ DPOAEs were obtained for the primary $\mathrm{f}_{2}$ frequencies at half octave distances $(4,5.7$, $8,11.3,16$ and $22.6 \mathrm{kHz})$. The $\mathrm{f}_{1} / \mathrm{f}_{2}$ ratio was 1.25 . The level of $\mathrm{f}_{1}\left(\mathrm{~L}_{1}\right)$ was increased in $5 \mathrm{~dB}$ steps from 40 to $80 \mathrm{~dB}$ SPL and the level of the accompanying $\mathrm{f}_{2}\left(\mathrm{~L}_{2}\right)$ was always $10 \mathrm{~dB}$ lower $\left(\mathrm{L}_{2}=\mathrm{L}_{1}-10\right)$. Each pair of primaries was presented 5 times and resulting spectra were averaged. DPOAE growth curves were obtained from both ears. The level of the noise floor at the frequency of the DPOAE was measured with the same procedure and using the same recording and stimulus set-up, immediately before the $2 f_{1}-f_{2}$ determination, but with the $f_{1}$ and $f_{2}$ switched off. 
CAP measurements were performed immediately after the DPOAE measurements. Electrocochleographic techniques were described elsewhere (Cappaert et al., 1999) and are only summarised here. Auditory evoked responses were recorded with a silver ball electrode at the apex of the cochlea. Trains of $8 \mathrm{~ms}$ tone bursts of 1 , $2,4,8,12,16$ and $24 \mathrm{kHz}$ were presented, with cosine-shaped rise and fall times of 1 $\mathrm{ms}$, except at $1 \mathrm{kHz}(2 \mathrm{~ms})$ and at $2 \mathrm{kHz}(1.5 \mathrm{~ms})$. The inter-stimulus interval was 99 $\mathrm{ms}$. The responses were amplified and band-pass filtered $(-12 \mathrm{~dB} /$ octave, $-3 \mathrm{~dB}$ at 1 $\mathrm{Hz}$ and $10 \mathrm{kHz}$ ). The averaged responses were saved for off-line analysis. The CAP amplitude was defined as the difference between the first negative peak and the summating potential in the electrophysiologic response.

\section{Hair cell counts}

Immediately after electrocochleography, the cochleas were fixed by transcardial perfusion with a chilled tri-aldehyde fixative consisting of 3\% glutaraldehyde, $2 \%$ formaldehyde, $1 \%$ acrolein, $2.5 \%$ dimethylsulfoxide in $0.1 \mathrm{M}$ sodium cacodylate buffer $\left(\mathrm{pH}\right.$ 7.4). This was followed by overnight immersion in the same fixative at $4^{\circ} \mathrm{C}$. The cochleas were processed further according to the routine method used for guinea pigs (De Groot et al., 1987). Two types of hair cell counts were performed. The first method, based on mid-modiolar sections, determines the number of hair cell bodies present at a limited number of locations along the basilar membrane and was performed in all animals. The second method quantifies the number of hair cells present along the entire length of the basilar membrane (cytocochleograms) but is based only on the presence of stereociliar bundles. The latter method was performed on a limited set of ears. We examined the right ears of 5 randomly selected animals in those groups which demonstrated synergistic effects with the first histological method.

\section{- Mid-modiolar sections}

In a mid-modiolar section $(1 \mu \mathrm{m})$ of the rat cochlea, the organ of Corti was examined at five locations, separated by a half-turn distance. Hair cell counts were performed at each of these five locations in both the left and the right cochlea, using 5-6 subsequent serial sections. If a hair cell is not seen in these 56 serial sections, it can be reliably assumed that the cell is not present. OHC 
loss was expressed as the percentage of remaining OHCs per cross-sectioned half turn, relative to the expected number of OHCs. No attempt was made to assess the degree of possible subcellular damage to surviving cells. Two investigators, independently of one another, performed all $\mathrm{OHC}$ counts in a single-blind fashion. An estimate was made of the characteristic frequencies of the locations along the basilar membrane at which the OHCs were counted (Müller, 1991). The estimated frequencies at the five examined locations are: $0.1,5,11,21$, and $51 \mathrm{kHz}$.

- Cytocochleograms

Orthogonal cuts were made between the coils of each half cochlea to separate it into six individual slices containing the half turns. The slices were mounted on microscope slides with epoxy resin, thinned with sanding disks and examined with a microscope equipped with differential interference contrast (Nomarski) optics. Cytocochleograms were established for each cochlea by plotting the percentage of missing inner hair cells and OHCs in each row against the distance of the basilar membrane as measured from the apex. The characteristic frequency correlate of each length of basilar membrane was estimated according to the place-frequency map for the rat (Müller, 1991).

\section{Data analysis and presentation}

In both the DPOAE recordings and the electrocochleopgraphic recordings, we acquired full amplitude growth curves at each frequency. For clarity, and to be able to compare these data directly with the histological data we needed a method of characterizing the amplitude growth curves with a single attribute. For the DPOAE measurements we chose an iso-response level at $3 \mathrm{~dB}$ SPL for 4, 5.7, 16 and $22.6 \mathrm{kHz}$ and at $10 \mathrm{~dB}$ SPL for 8 and $11.3 \mathrm{kHz}$ because at these emission levels, the emission was unequivocally above the noise level. Stimulus levels needed to evoke the required emission levels were between 60 and $80 \mathrm{~dB}$ SPL in the control groups (the $\mathrm{L}_{1}$ levels in the control group were $68.8,66.4,62.5,63.4,76.6,63.4 \mathrm{~dB}$ SPL for 4, 6, 8, 12, 16 and $24 \mathrm{kHz}$, respectively). In relation to these stimulus levels, a CAP iso-response level was set at $10 \mu \mathrm{V}$ at $1,2,4,8$ and $12 \mathrm{kHz}$ and a $3 \mu \mathrm{V}$ iso-response level was used at 16 and $24 \mathrm{kHz}$. This resulted in required sound levels comparable to those for the DPOAEs given above (the average stimulus level needed to evoke the CAP iso- 
response levels in the control group were 80.3, 76.9, 63.1, 59.8, 74.3, 47.9, $61 \mathrm{~dB}$ SPL for $1,2,4,8,12,16$ and $24 \mathrm{kHz}$, respectively). In the figures, iso-response levels in the exposed groups were presented as shifts with respect to the levels from the control groups (averaged over right and left ear and pooled for all three cohorts, $n=24 \times 2$ ears). Statistical analysis was performed with the STATISTICA package. ANOVA was based on ethyl benzene concentration and noise level as between-subject factors and frequency as a within-subject factor. When appropriate, post hoc Tukey's HSD test was used to further investigate the differences. In addition, we checked for addititvity in the combined exposure of the effects due to noise and ethyl benzene separately by testing whether of not the sum of the separate effects minus the effects of the combined exposure differs significantly from zero (null hypothesis: $\mathrm{OHCloss}_{\text {(ethylbenzene) }}+\mathrm{OHCloss}_{\text {(noise) }}-\mathrm{OHCloss}_{\text {(combination) }}=0$ ).

\section{RESUlTS}

\section{General}

The rats exhibited no overt signs of ill health during and after the exposure. The body weight of the exposed animals did not differ from the weight of the animals in the control group at the end of the exposure period.

\section{DPOAEs}

The average shifts in DPOAE iso-response levels as a function of stimulus frequency $\left(f_{2}\right)$ are shown in Fig. 2 for all 8 experimental conditions. As expected on the basis of the choices we made for the exposure levels, the shifts are quite modest, within + and $-10 \mathrm{~dB}$ under most conditions.

ANOVA revealed significant interactions between ethyl benzene and noise $\left(F_{(4,76)}=4.4, P<0.01\right)$, between noise and frequency $\left(F_{(10,380)}=4.7, P<0.01\right)$ and between ethyl benzene, noise and frequency $\left(\mathrm{F}_{(20,380)}=4.0, \mathrm{P}<0.01\right)$. Post hoc Tukey's HSD test was used to further examine significance of the differences. The probabilities of these tests, collapsed over frequencies, are shown in Table 2. The only significant differences between the control group and the exposed groups are found at a $105 \mathrm{~dB}$ SPL noise level (with and without 400 ppm ethyl benzene exposure). 

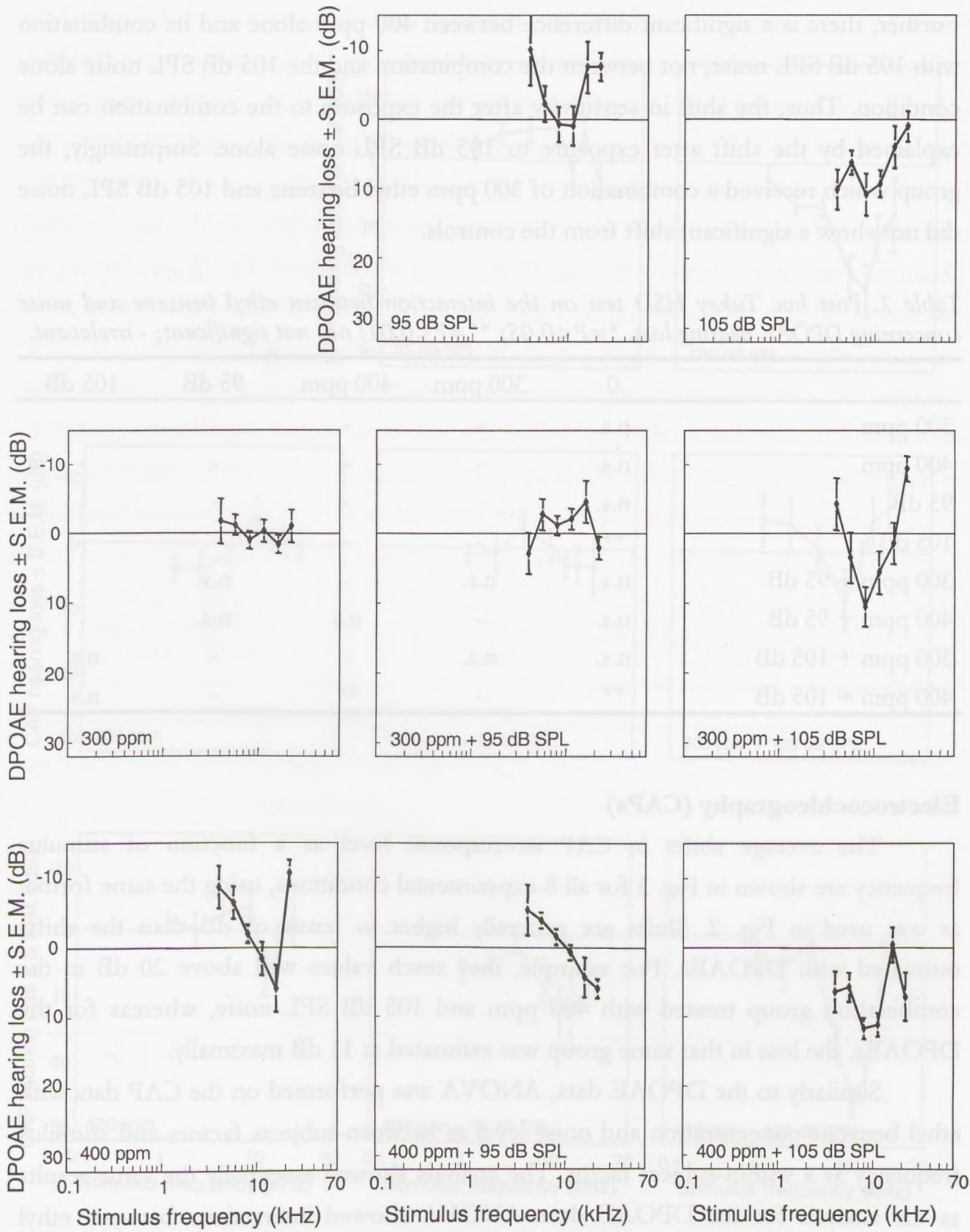

Figure 2. Average DPOAE hearing loss \pm S.E.M. as a function of frequency. Each graph represents the results for a certain type of exposure. Ethyl benzene concentration (ppm) and/or noise level (dB SPL) are indicated in each graph. Shown are $d B$ shifts in iso-response level relative to the average iso-response level in the control group. Hearing loss is plotted downward. 
Further, there is a significant difference between $400 \mathrm{ppm}$ alone and its combination with $105 \mathrm{~dB}$ SPL noise, not between the combination and the $105 \mathrm{~dB}$ SPL noise alone condition. Thus, the shift in sensitivity after the exposure to the combination can be explained by the shift after exposure to $105 \mathrm{~dB}$ SPL noise alone. Surprisingly, the group which received a combination of $300 \mathrm{ppm}$ ethyl benzene and $105 \mathrm{~dB}$ SPL noise did not show a significant shift from the controls.

Table 2. Post hoc Tukey HSD test on the interaction between ethyl benzene and noise concerning DPOAE hearing loss. ${ }^{*}=P<0.05 ;{ }^{* *}=P<0.01$; n.s. not significant; - irrelevant.

\begin{tabular}{lccccc}
\hline & 0 & $300 \mathrm{ppm}$ & $400 \mathrm{ppm}$ & $95 \mathrm{~dB}$ & $105 \mathrm{~dB}$ \\
\hline $300 \mathrm{ppm}$ & n.s. & - & - & - & - \\
$400 \mathrm{ppm}$ & n.s. & - & - & - & - \\
$95 \mathrm{~dB}$ & n.s. & - & - & - & - \\
$105 \mathrm{~dB}$ & $* *$ & - & - & - & - \\
$300 \mathrm{ppm}+95 \mathrm{~dB}$ & n.s. & n.s. & - & n.s. & - \\
$400 \mathrm{ppm}+95 \mathrm{~dB}$ & n.s. & - & n.s & n.s. & - \\
$300 \mathrm{ppm}+105 \mathrm{~dB}$ & n.s. & n.s. & - & - & n.s. \\
$400 \mathrm{ppm}+105 \mathrm{~dB}$ & $* *$ & - & $* *$ & - & n.s. \\
\hline
\end{tabular}

\section{Electrocochleography (CAPs)}

The average shifts in CAP iso-response level as a function of stimulus frequency are shown in Fig. 3 for all 8 experimental conditions, using the same format as was used in Fig. 2. Shifts are generally higher in terms of $d B$ than the shifts estimated with DPOAEs. For example, they reach values well above $20 \mathrm{~dB}$ in the combination group treated with $400 \mathrm{ppm}$ and $105 \mathrm{~dB}$ SPL noise, whereas for the DPOAEs, the loss in that same group was estimated at $11 \mathrm{~dB}$ maximally.

Similarly to the DPOAE data, ANOVA was performed on the CAP data with ethyl benzene concentration and noise level as between-subjects factors and stimulus frequency as a within-subject factor. The analysis showed essentially the same results as the analysis for the DPOAE data. ANOVA showed interactions between ethyl benzene and noise $\left(\mathrm{F}_{(4,76)}=3.7, \mathrm{P}<0.01\right)$, between ethyl benzene and frequency $\left(\mathrm{F}_{(12,456)}=3.9, \mathrm{P}<0.01\right)$ and between noise and frequency $\left(\mathrm{F}_{(12,456)}=7.4, \mathrm{P}<0.01\right)$. The higher interaction between ethyl benzene, noise and frequency was not significant. 

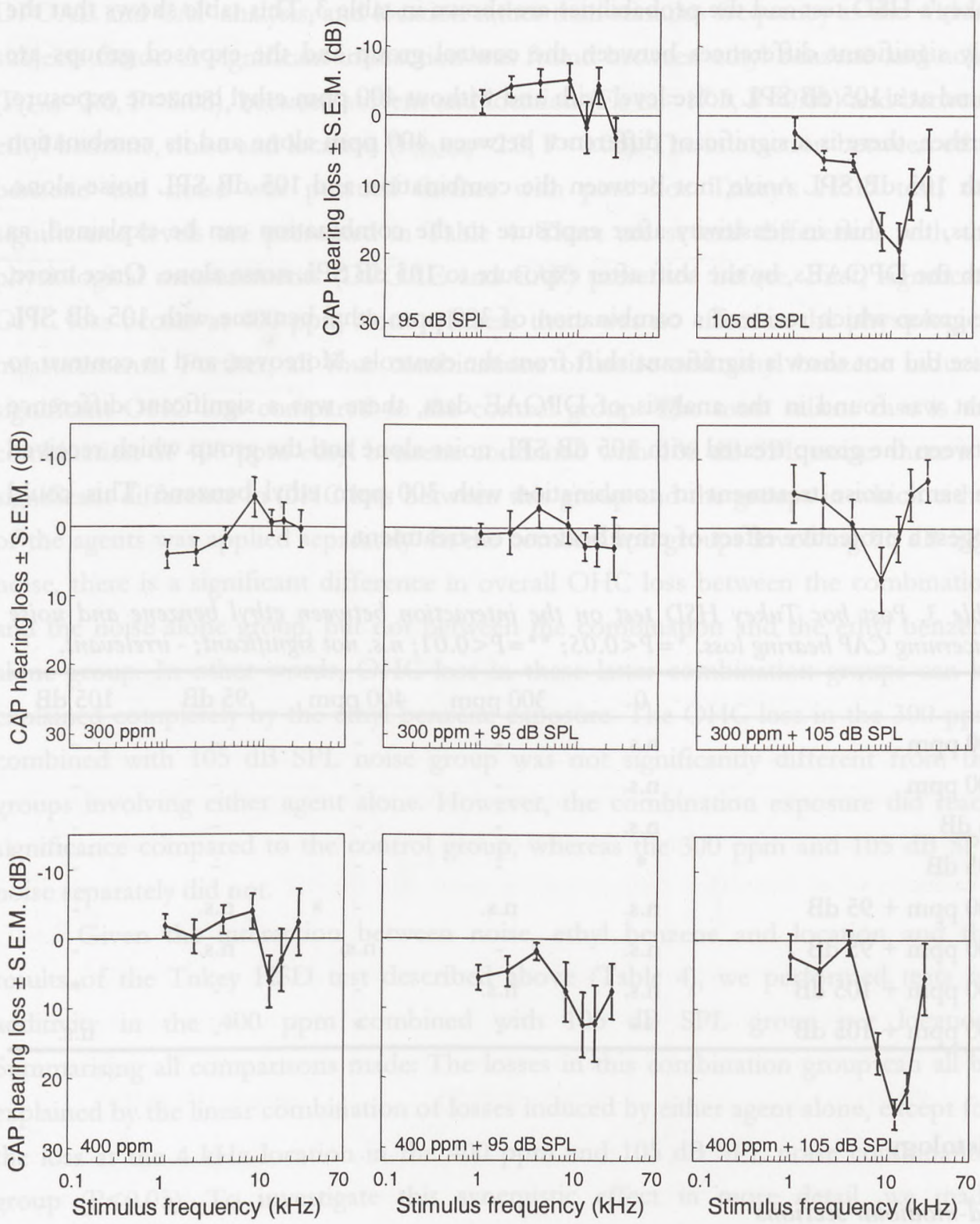

Figure 3. Average CAP hearing loss \pm S.E.M. as a function of frequency. Each graph represents the results for a certain type of exposure. Ethyl benzene concentration (ppm) and/or noise level (dB SPL) are indicated in each graph. Shown are dB shifts in iso-response level relative to the average iso-response level in the control group. Hearing loss is plotted downward. 
The interaction between ethyl benzene and noise was further pursued by post hoc Tukey's HSD test and the probabilities are shown in table 3 . This table shows that the only significant differences between the control group and the exposed groups are found at a $105 \mathrm{~dB}$ SPL noise level with and without 400 ppm ethyl benzene exposure. Further, there is a significant difference between $400 \mathrm{ppm}$ alone and its combination with $105 \mathrm{~dB}$ SPL noise, not between the combination and $105 \mathrm{~dB}$ SPL noise alone. Thus, the shift in sensitivity after exposure to the combination can be explained, as with the DPOAEs, by the shift after exposure to $105 \mathrm{~dB}$ SPL noise alone. Once more, the group which received a combination of $300 \mathrm{ppm}$ ethyl benzene with $105 \mathrm{~dB}$ SPL noise did not show a significant shift from the controls. Moreover, and in contrast to what was found in the analysis of DPOAE data, there was a significant difference between the group treated with $105 \mathrm{~dB}$ SPL noise alone and the group which received the same noise treatment in combination with $300 \mathrm{ppm}$ ethyl benzene. This could suggest a protective effect of ethyl benzene co-treatment.

Table 3. Post hoc Tukey HSD test on the interaction between ethyl benzene and noise concerning CAP hearing loss. ${ }^{*}=P<0.05 ;{ }^{* *}=P<0.01$; n.s. not significant; - irrelevant.

\begin{tabular}{lccccc}
\hline & 0 & $300 \mathrm{ppm}$ & $400 \mathrm{ppm}$ & $95 \mathrm{~dB}$ & $105 \mathrm{~dB}$ \\
\hline $300 \mathrm{ppm}$ & n.s. & - & - & - & - \\
$400 \mathrm{ppm}$ & n.s. & - & - & - & - \\
$95 \mathrm{~dB}$ & n.s. & - & - & - & - \\
$105 \mathrm{~dB}$ & $*$ & - & - & - & - \\
$300 \mathrm{ppm}+95 \mathrm{~dB}$ & n.s. & n.s. & - & n.s. & - \\
$400 \mathrm{ppm}+95 \mathrm{~dB}$ & n.s. & - & n.s. & n.s. & - \\
$300 \mathrm{ppm}+105 \mathrm{~dB}$ & n.s. & n.s. & - & - & $*$ \\
$400 \mathrm{ppm}+105 \mathrm{~dB}$ & ** & - & $*$ & - & n.s. \\
\hline
\end{tabular}

\section{Histology}

\section{Mid-modiolar sections}

The average $\mathrm{OHC}$ loss as a function of estimated best frequency for the examined locations in the midmodiolar sections is presented in Fig. 4. Each point is based on all three $\mathrm{OHC}$ rows combined. Each panel represents one experimental condition. Inner hair cell loss was never seen. 
ANOVA was used for statistical evaluation with the same set-up as for the DPOAE and CAP analysis, and location rather than stimulus frequency as the withinsubjects factor. A significant interaction was found between ethyl benzene and noise $\left(F_{(4,76)}=3.6, P<0.05\right)$, between solvent and location $\left(F_{(8,304)}=17.5, P<0.01\right)$ and between ethyl benzene, noise and location $\left(\mathrm{F}_{(16,304)}=2.0, \mathrm{P}<0.05\right)$. The interaction between ethyl benzene and noise was pursued further with post hoc Tukey's HSD test; the significance levels are presented in Table 4. There are several differences with the physiological measurements (DPOAE and CAP) presented before. First, significant $\mathrm{OHC}$ loss occurs at $400 \mathrm{ppm}$ alone whereas there was no effect in the physiological measurements. Further, all four combinations of noise and ethyl benzene induced significant OHC loss compared to the control group. The most salient case is the combination of 400 ppm ethyl benzene combined with $105 \mathrm{~dB}$ SPL noise: there is a significant difference in $\mathrm{OHC}$ loss between this group and the groups in which either of the agents was applied separately. In the combination groups involving $95 \mathrm{~dB} \mathrm{SPL}$ noise, there is a significant difference in overall OHC loss between the combination and the noise alone group, but not between the combination and the ethyl benzene alone group. In other words, OHC loss in these latter combination groups can be explained completely by the ethyl benzene exposure. The OHC loss in the $300 \mathrm{ppm}$ combined with $105 \mathrm{~dB}$ SPL noise group was not significantly different from the groups involving either agent alone. However, the combination exposure did reach significance compared to the control group, whereas the $300 \mathrm{ppm}$ and $105 \mathrm{~dB}$ SPL noise separately did not.

Given the interaction between noise, ethyl benzene and location and the results of the Tukey HSD test described above (Table 4), we performed tests on additivity in the $400 \mathrm{ppm}$ combined with $105 \mathrm{~dB}$ SPL group per location. Summarising all comparisons made: The losses in this combination group can all be explained by the linear combination of losses induced by either agent alone, except for the loss at the $4 \mathrm{kHz}$ location in the $400 \mathrm{ppm}$ and $105 \mathrm{~dB}$ SPL noise combination group $(\mathrm{P}<0.05)$. To investigate this synergistic effect in more detail, we made cytocochleograms in a subset of the available cochleas, also including the $300 \mathrm{ppm}$ with and without $105 \mathrm{~dB}$ SPL noise groups. 

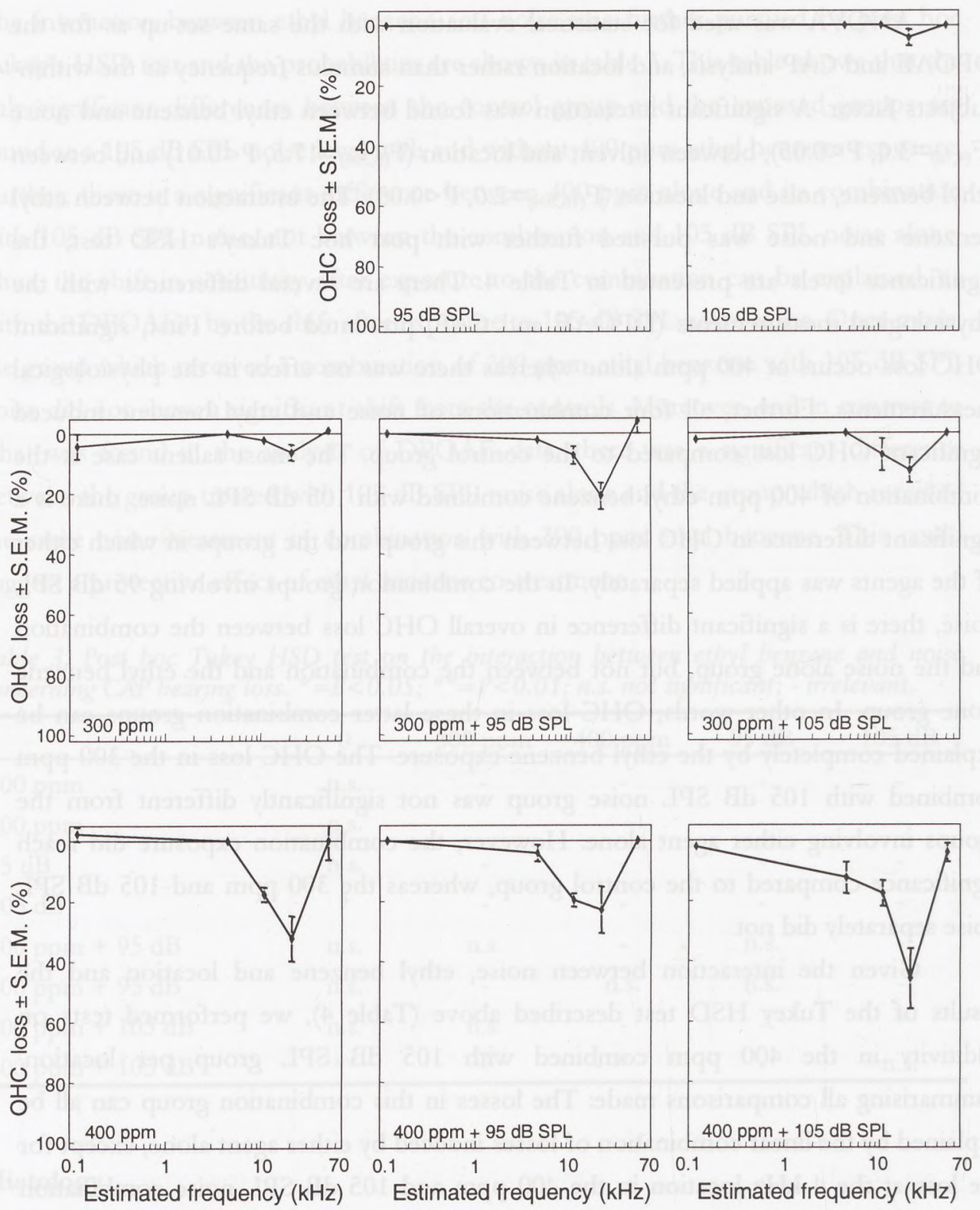

Figure 4. Average OHC loss \pm S.E.M. at 5 locations. Counts were performed in midmodiolar sections of the cochlea in which the organ of Corti was examined at 5 specific locations separated by a half turn. The frequencies of these locations, which were used to construct the horizontal axis in each graph, were estimated from the place-frequency map of Müller (1991). Ethyl benzene concentration ( $p p m)$ and/or noise level (dB SPL) are indicated in each graph. OHC loss is plotted downward. 
Table 4. Post hoc Tukey HSD test on the interaction between ethyl benzene and noise concerning OHC loss (based on midmodiolar sections). ${ }^{*}=P<0.05 ;{ }^{* *}=P<0.01$; n.s. not significant; - irrelevant.

\begin{tabular}{lccccc}
\hline & 0 & $300 \mathrm{ppm}$ & $400 \mathrm{ppm}$ & $95 \mathrm{~dB}$ & $105 \mathrm{~dB}$ \\
\hline $300 \mathrm{ppm}$ & n.s. & - & - & - & - \\
$400 \mathrm{ppm}$ & $* *$ & - & - & - & - \\
$95 \mathrm{~dB}$ & n.s. & - & - & - & - \\
$105 \mathrm{~dB}$ & n.s. & - & - & - & - \\
$300 \mathrm{ppm}+95 \mathrm{~dB}$ & $* *$ & n.s. & - & n.s. & - \\
$400 \mathrm{ppm}+95 \mathrm{~dB}$ & $* *$ & - & n.s. & $* *$ & - \\
$300 \mathrm{ppm}+105 \mathrm{~dB}$ & $*$ & n.s. & - & - & n.s. \\
$400 \mathrm{ppm}+105 \mathrm{~dB}$ & $* *$ & - & $* *$ & - & $* *$ \\
\hline
\end{tabular}

\section{Cytocochleograms}

Cytocochleograms are presented in Fig. 5 for the right ears of 5 randomly chosen animals in the groups treated with both concentrations of ethyl benzene with and without the highest level (105 dB SPL) of noise. The results were averaged across each experimental group of animals. Evidently, there is a clear difference between the effects on the different rows of hair cells. First, the row of inner hair cells is not affected by either agent. Further, ethyl benzene affects primarily the third outermost row of OHCs, than the second row and least of all the first row. The effect in terms of hair cell presence of $105 \mathrm{~dB}$ SPL noise is very modest and seems to be confined to the first row of OHCs.

ANOVA on this data set showed significant interactions between all 4 factors, ethyl benzene concentration, noise level, hair cell row and location. Tukey's HSD test was performed on solvent concentration and noise level (hair cell rows and location collapsed). The results are presented in Table 5 and reveal in more detail the effects of both agents on OHC loss. In both combination groups the OHC losses in the combination groups are significantly larger than in the groups exposed to either agent alone. Thus, we had justification to perform the test on additivity of losses induced by agents separately and performed this test per location and per OHC row. The results are presented in Table 6 . 

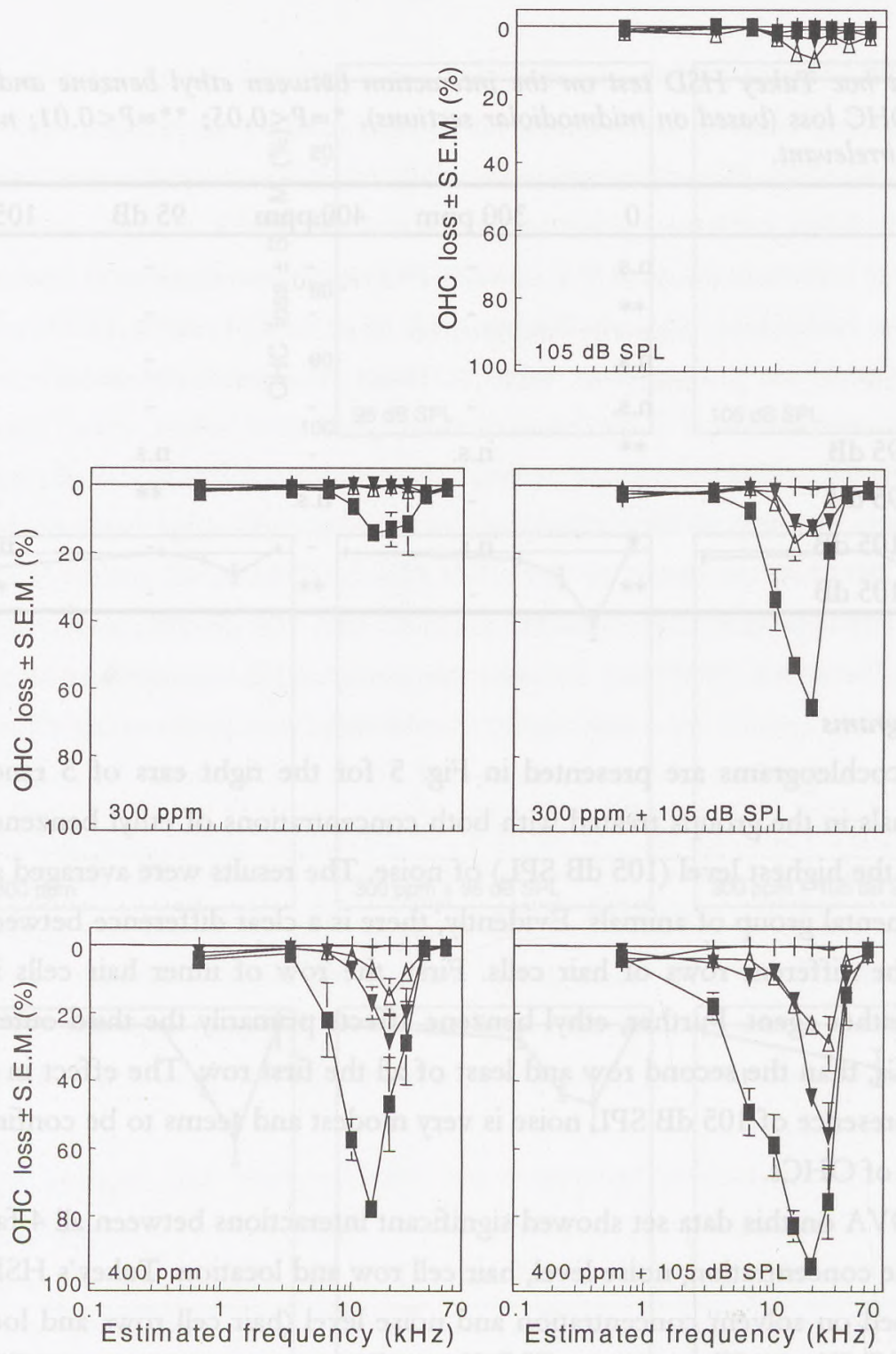

Figure 5. Average cytocochleograms of rats $(5$ randomly selected cochleas per condition) Counts were performed along the entire length of the basilar membrane, divided into sections of about $1 \mathrm{~mm}$. The characteristic frequency of the point on the basilar membrane in the middle of each section, which was used to construct the frequency axis in each graph, was estimated from the place-frequency map of Müller (1991). Ethyl benzene concentration ( $p p m)$ and/or noise level (dB SPL) are indicated in each graph. Hair cell loss is plotted downward for each hair cell row separately. The average percentage of hair cell loss is presented as a function of frequency. IHC: inner hair cells (+); OHC1: first or inner row of OHCs $(\triangle)$; $\mathrm{OHC2}$ : second row of $\mathrm{OHCs}(\boldsymbol{\nabla})$; OHC3: third or outer row of $\mathrm{OHCs}$ (a). 
In the combination group with $300 \mathrm{ppm}$ ethyl benzene, synergistic interactions occurred in all three rows in the mid-frequency region. This region seemed to become wider going from the first row to the third row. In the combination group with 400 ppm, the situation was different. As might be expected with the higher ethyl benzene exposure, the interaction region broadened further, but synergistic interactions as defined in our model on additivity disappeared in the middle of this region. Thus, synergistic interactions occurred preferably on the edges of the affected region.

Table 5. Post hoc Tukey HSD test on the interaction between ethyl benzene and noise concerning hair cell loss (based on cytocochleograms but collapsed over hair cell row and frequency). ${ }^{*}=P<0.05 ;{ }^{*}=P<0.01$; n.s. not significant; - irrelevant.

\begin{tabular}{lcccc}
\hline & 0 & 300 ppm & 400 ppm & $105 \mathrm{~dB}$ SPL \\
\hline $300 \mathrm{ppm}$ & n.s. & - & - & - \\
$400 \mathrm{ppm}$ & $* *$ & - & - & - \\
$105 \mathrm{~dB} \mathrm{SPL}$ & n.s. & - & - & - \\
$300 \mathrm{ppm}+105 \mathrm{~dB} \mathrm{SPL}$ & $* *$ & $* *$ & - & $* *$ \\
$400 \mathrm{ppm}+105 \mathrm{~dB}$ SPL & $* *$ & - & $* *$ & $* *$ \\
\hline
\end{tabular}

Table 6. Synergy test on cytococleogram data per $\mathrm{OHC}$ row. ${ }^{*}=P<0.05$, ${ }^{* *}=P<0.01$; n.s.: not significant (null hypothesis accepted).

null hypothesis: $\mathrm{OHCloss}_{(\text {ethyl benzene) }}+\mathrm{OHCloss}_{(\text {noise })}-\mathrm{OHCloss_{( \text {combination } ) }}=0$

\begin{tabular}{llllllllll}
\hline Location & $1 \mathrm{kHz}$ & $3 \mathrm{kHz}$ & $7 \mathrm{kHz}$ & $10 \mathrm{kHz}$ & $14 \mathrm{kHz}$ & $20 \mathrm{kHz}$ & $26 \mathrm{kHz}$ & $36 \mathrm{kHz}$ & $53 \mathrm{kHz}$
\end{tabular}

\section{$300 \mathrm{ppm}+105 \mathrm{~dB}$ SPL}

$\begin{array}{llllllllll}\mathrm{OHC} 1 & \text { n.s. } & \text { n.s. } & \text { n.s. } & \text { n.s. } & * & * & \text { n.s. } & \text { n.s. } & \text { n.s. } \\ \mathrm{OHC2} & \text { n.s. } & \text { n.s. } & \text { n.s. } & \text { n.s. } & * * & * * & * * & \text { n.s. } & \text { n.s. } \\ \text { OHC3 } & \text { n.s. } & \text { n.s. } & * * & * * & * * & * * & \text { n.s. } & \text { n.s. } & \text { n.s. }\end{array}$

\section{$400 \mathrm{ppm}+105 \mathrm{~dB}$ SPL}

\begin{tabular}{cccccccccc}
$\mathrm{OHC} 1$ & n.s. & n.s. & n.s. & n.s. & n.s. & n.s. & $* *$ & n.s. & n.s. \\
$\mathrm{OHC} 2$ & n.s. & $* *$ & $*$ & n.s. & n.s. & $*$ & $* *$ & $* *$ & n.s. \\
$\mathrm{OHC} 3$ & n.s. & $* *$ & $* *$ & n.s. & n.s. & $* *$ & $* *$ & $*$ & n.s. \\
\hline
\end{tabular}




\section{DIsCUSSION}

\section{Effects of ethyl benzene}

Ethyl benzene alone, applied at 300 and $400 \mathrm{ppm}$, did not cause any significant DPOAE or CAP hearing loss (Figs. 2 and 3; Tables 2 and 3). Based on a previous study in our laboratory (Chapter 3), we expected a small effect after $400 \mathrm{ppm}$ ethyl benzene. The simplest explanation for this slight inconsistency is experimental variability. Another explanation might be that we condensed the entire growth curves into an iso-response level in this study whereas in the previous study we used the entire growth curve. Consistent with the previous study, ethyl benzene did cause OHC loss (Figs. 4 and 5; Tables 4 and 5). The OHC loss is located in the midfrequency region of the cochlea, as reported before for ethyl benzene (Cappaert et al., 1999; Chapters 2 and 3) and other organic solvents (Johnson and Canlon, 1994b; Yano et al., 1992; Lataye and Campo, 1997; Lataye et al., 2000). The cytocochleograms (Fig. 5) showed that ethyl benzene exposure evokes OHC loss specifically in the third row. With increasing ethyl benzene concentration, the affected region broadens, the loss becomes larger and the loss expands to the second and first row of OHCs. This pattern of $\mathrm{OHC}$ loss has also been reported for other organic solvents like toluene (Pryor et al., 1984; Sullivan et al., 1987; Johnson and Canlon, 1994b; Lataye and Campo, 1997) and styrene (Mäkitie, 1997; Loquet et al., 1999; Lataye et al., 2000). It is interesting to note that this particular pattern of damage, the third row of OHCs affected first, has also been observed in animal models of another major inner ear disease, presbyacusis. Studies in aging animals showed that the third row of OHCs is the first row that shows signs of pathology (guinea pig: Coleman, 1976; chinchilla: Bohne et al., 1990; rat: Borg and Viberg, 1987; Keithley and Feldman, 1982; gerbil: Tarnowski et al., 1991).

The effects after mild ethyl benzene exposure can be characterized as severe where $\mathrm{OHC}$ loss is concerned and minor with respect to physiological deterioration. Apparently, there can be a relatively large loss of OHCs, especially in the third row, without any functional consequences. This observation of the relative unimportance of the third row of OHCs for cochlear sensitivity is in line with reports emphasizing the relative importance of the first row of OHCs (Liberman, 1987; Yoshida and Liberman, 1999). 


\section{Noise exposure}

The situation after noise exposure can be described as the mirror image of the situation after ethyl benzene exposure. The highest noise exposure in the present study had a profound effect on the physiological data, but caused only minor OHC loss. Both the DPOAE and CAP data showed hearing loss after $105 \mathrm{~dB}$ noise alone but not, as expected, after $95 \mathrm{~dB}$ SPL noise. Only minor OHC loss was detected in the mid-modiolar sections, where detection of OHCs is based on the presence of the cell bodies (Fig. 4). The cytocochleograms (Fig. 5), which are based on detection of stereocilia, confirmed the minor loss. This loss was found exclusively in the first row of OHCs. These results, functional loss without accompanying hair cell loss, are in line with our previous results (Chapter 4) and results of others (Engström, 1984; Borg et al., 1995; Lataye and Campo, 1997; Lataye et al., 2000). The simplest explanation for this apparent discrepancy between physiological and morphological data is that subcellular damage, e.g., stereociliar damage has occurred (Engström, 1984; Lataye and Campo, 1997; Lataye et al., 2000). Stereociliar injuries are not detectable with the morphological techniques used in this study, but they can explain the DPOAE and CAP hearing loss found after $105 \mathrm{~dB}$ SPL noise exposure. An alternative explanation would be an effect on the stria vascularis which gives rise to the endocochlear potential (EP) but this alternative seems rather far-fetched, because of the frequency dependence of the hearing loss and because previous authors failed to find a longterm effect of noise on the EP (Melichar et al., 1980; Syka et al., 1981).

\section{Combination exposure}

Our physiological measurements (DPOAE and CAP) did not detect any significant synergistic interactions between noise and ethyl benzene exposure (Figs. 2 and 3; Tables 2 and 3). In all cases the effects, when present, could be explained by the effects of noise exposure alone. In contrast, other studies concerning combination exposures with organic solvents and noise generally found a hearing loss that exceeded the summated loss (in dB) caused by either agent alone (Johnson et al., 1988; Lataye and Campo, 1997; Lataye et al., 2000; Muijser et al., 2000). A general characteristic of the latter studies is that they used relatively high concentrations of solvent, compared to the concentrations used in this study. As mentioned before, our goal was to investigate interactions between agents applied at liminally effective levels. As we did find synergistic interactions in the morphological data, it is possible that we 
would have found synergistic interactions in the physiological data as well, when we would have used higher exposure levels or more animals.

Considering the OHCs clear synergetic interactions were found (Figs. 4 and 5; Tables 4-6). Synergetic interactions occurred especially in the third row of OHCs, but could also be found in the first and second row. The spatial pattern of synergistic interactions differed slightly between the two combination groups. In the $300 \mathrm{ppm}$ ethyl benzene combined with $105 \mathrm{~dB}$ SPL noise exposure group, these interactions were found at those frequencies where OHC loss was maximal in the ethyl benzene alone group. In the $400 \mathrm{ppm}$ combined with $105 \mathrm{~dB}$ group, there was a tendency for synergetic interactions to occur at the edges of the area where OHC loss in the ethyl benzene alone group was maximal. Our results are generally in line with the only other interaction studies with rats in which hair cell counts were performed. Lataye and Campo (1997) indicated that OHC loss in a group exposed to a combination of toluene and noise exceeded the loss in the toluene alone group. As there was no OHC loss in the noise alone group, they took this to be the result of a synergistic interaction. Lataye et al. (2000) reported similar results for the combination of styrene with noise.

\section{Mechanism of synergy}

Synergetic interactions as occurred particularly in the third row of OHCs can be expected to occur when two agents have different mechanisms of action. In this case, the mechanism of action of noise seems to be known (Engström, 1984; Borg et al., 1995; Lataye and Campo, 1997; Lataye et al., 2000) and can be described as sublethal micro-disturbances in hair cells. The mechanism of action of organic solvents is less known, but must involve some kind of lethal physical or chemical reaction in hair cells. The difference in hair cell mortality after both agents implies that they have a different mechanism of action. Apparently, when combined, the action of one agent facilitates the action of the other. For instance, it is possible that micro-disturbances in hair cells due to noise facilitate the entry of ethyl benzene. Intracellular calcium might be important here. There are indications from separate in vitro experiments that both the organic solvent toluene (Liu and Fechter, 1997) and mechanical disturbances mimicking noise exposure in vivo (Fridberger and Ulfendahl, 1996) increase intracellular calcium. The combination might enhance intracellular calcium 
concentrations from sub-lethal to lethal levels. However, within the context of the present study it is impossible to make more definite statements about the mechanism of action. A more cellular approach involving combination exposure might shed more light on this issue.

\section{Relations between methods to assess hearing loss}

In the previous paragraphs we have discussed the poor relation between $\mathrm{OHC}$ loss and physiologically evaluated loss repeatedly. Other authors have observed similar discrepancies between evoked potential measurements and morphology after noise exposure (Engström, 1984; Borg et al., 1995; Lataye and Campo, 1997; Lataye et al., 2000). Further, a poor correspondence between DPOAE hearing loss and OHC loss was reported before by Subramaniam et al. (1995) in chinchillas after noise exposure. The relation between the CAP data and the DPOAE data was qualitatively consistent, in the sense that they both showed significant losses in the same groups. Quantitatively however, the loss assessed with DPOAEs was always smaller than the loss assessed with CAP measurements. Possibly, the CAP, being a neural response but depending on hair cell integrity, experienced additional damage, either directly from ethyl benzene or from excitotoxic damage after noise exposure. The latter possibility, however, seems remote because excitotoxicity should have vanished at the time of CAP recording (Robertson, 1983; Puel et al., 1998).

\section{Conclusions and implications for occupational safety}

The physiological measurements, DPOAEs and CAPs, did not show synergistic interactions between noise and ethyl benzene exposure at the levels applied in this study. However, we found clear synergistic interactions in the morphological data, especially in the third row of OHCs. These seemingly conflicting results make it rather difficult to draw definite conclusions. Of course, it is more important to protect hearing sensitivity than to protect the third row of OHCs, which does not seem important for cochlear sensitivity anyway, at least in the rat. Thus, our results could be interpreted to imply that risks encountered by workers exposed to ethyl benzene and noise are not very serious. However, we only tested a very basic attribute of the auditory system, i.e., cochlear sensitivity. It is possible that damage to the third row could have an effect on for instance frequency selectivity. Moreover, we also saw 
some synergistic interactions in the first and second row, which are more important for cochlear sensitivity than the third row in the rat. Further, as mentioned before, higher exposure levels and/or longer exposure durations might be accompanied by more widespread synergistic effects, also on the sensitivity.

\section{ACKNOWLEDGEMENTS}

The authors wish to thank R. Bulder, J.C.M.J de Groot and F. Hendriksen for their histological assistance and E. Duistermaat for his technical assistance with the ethyl benzene exposures. This study was supported by grants of NWO-MW (grant number 9004-63-085) and by the Heinsius-Houbolt Fund. 


\section{RATS ARE HIGHLY SUSCEPTIBLE TO THE ORGANIC SOLVENT ETHYL BENZENE WHEREAS GUINEA PIGS ARE NOT}

Ethyl benzene affects auditory sensitivity by interfering with outer hair cell function in rats. The present study was designed to compare the ototoxic effects of ethyl benzene in the guinea pig with the effects in rats. Guinea pigs and rats were placed in inhalation chambers and exposed to either ethyl benzene or control conditions after which electrocochleography and histology were performed. Auditory thresholds deteriorated considerably in the mid-frequency range $(8-20 \mathrm{kHz})$ in rats exposed to ethyl benzene $(550$ ppm, 8 hours/day, 5 days). Outer hair cell loss was found in corresponding cochlear regions. Guinea pigs, in contrast, showed no threshold shifts and no outer hair cell loss after exposure to much higher ethyl benzene levels ( $2500 \mathrm{ppm} ; 6$ hours/day; 5 days). A limited study ( 4 rats; 4 guinea pigs) was performed in an attempt to understand these differences in susceptibility. We determined the ethyl benzene concentration in blood in both species after exposure to 500 ppm ethyl benzene ( 8 hours/day, 3 days) using gas chromatography. At the end of day one, blood of the rats contained $23.2 \pm 0.8 \mathrm{ng} / \mathrm{ml}$ ethyl benzene. In contrast, the concentration of ethyl benzene in guinea pig blood was $2.8 \pm 0.1$ $\mathrm{ng} / \mathrm{ml}$. Although, the ethyl benzene concentration in both species had decreased after three days of exposure, the ethyl benzene concentration in rat blood was still 4.3 times the concentration in guinea pig blood. Thus, it seems that the difference in susceptibility can be explained by differences in uptake, distribution, metabolism and/or excretion of ethyl benzene between rats and guinea pigs.

(Submitted; co-authors: Klis, Muijser, Kulig, Ravensberg, Smoorenburg) 


\section{INTRODUCTION}

Some organic solvents are known to affect the auditory system in mammals. Several studies reported that humans who were occupationally exposed to organic solvents like toluene (Morata et al., 1993), styrene (Muijser et al., 1988; Möller et al., 1990; Rebert and Hall, 1994) and trichloroethylene (see Morata et al., 1994 for review) can develop hearing deficits. Animal experiments have confirmed that several organic solvents do affect the auditory system and have shown that the auditory periphery is the main target for these solvents. Rats exposed to toluene, styrene, trichloroethylene or xylene showed hearing loss predominantly in the mid-frequency region, which is from 8-20 kHz in the rat (e.g., Pryor et al., 1983; Pryor et al., 1987; Jaspers et al., 1993 and Pryor et al., 1987, respectively).

However, organic solvent ototoxicity seems to be species dependent. Guinea pigs did not show any ototoxic effects as a result of exposure to solvents known to be ototoxic in rats. To our knowledge, four ototoxicity studies with organic solvents were performed on guinea pigs. Yamamura and colleagues published the first organic solvent study with this species in 1983. They exposed four groups of albino guinea pigs to $0,6000,12000$ and $17000 \mathrm{ppm}$ trichloroethylene, 4 hours/day for 5 days. The compound action potential (CAP) in response to a $7 \mathrm{kHz}$ tone and the cochlear microphonics $(\mathrm{CM})$ at $4 \mathrm{kHz}$ were recorded. Both potentials did not differ significantly between the control group and all three exposed groups. Pryor and Rebert (1993) exposed pregnant guinea pigs to toluene. During the exposure period, they increased the toluene concentration from 500 up to $2000 \mathrm{ppm}$ but never observed hearing impairment in neither mothers nor their offspring. Subsequently, the male offspring was exposed to further increasing toluene vapor concentrations (2000 to $4000 \mathrm{ppm}$ over a 21 day period). Again, no hearing loss was detected after the exposure. In an acute study, Fechter (1993) exposed pigmented guinea pigs to 500 $\mathrm{ppm}$ and $1200 \mathrm{ppm}$ styrene for 7 hours. The CAP and CM in response to tone bursts from $2-40 \mathrm{kHz}$ were assessed $18-22$ hours after the end of the exposure. No differences were found between the control group and both (500 and $1200 \mathrm{ppm}$ ) treated groups. Toluene was used as organic solvent in the fourth study (Campo et al., 1993). Electrocochleography in the frequency range from $2-32 \mathrm{kHz}$ did not show any effect of $1000 \mathrm{ppm}$ toluene (6 hours/day, 14 days). Furthermore, no histological changes (SEM on surface preparations) were detected. 
A previous study in our laboratory showed that ethyl benzene is a very potent ototoxicant in rats (Cappaert et al., 1999). Exposure to 800 ppm ethyl benzene for 8 hours/day for 5 days induced a hearing loss of 10 to $30 \mathrm{~dB}$ over the entire frequency range tested (1-24 kHz). Concomitantly, an outer hair cell (OHC) loss was found, which was confined to the upper basal and lower middle turns (estimated frequency range: $11-21 \mathrm{kHz}$ ). Triggered by this high ototoxicity of ethyl benzene in rats, we decided to evaluate the ototoxic potency of this compound in guinea pigs. Using electrocochleography and histological examination of the cochlea, we found, consistent with the studies described above, that hearing was affected in rats, but not in guinea pigs. Hence an impressive difference in susceptibility between both species was found: guinea pigs were not susceptible at all. In an attempt to explain this difference, we performed a limited study on the concentration of ethyl benzene in blood of rats and guinea pigs after exposure to ethyl benzene.

\section{METHODS}

\section{Experiment 1: Ototoxicity of ethyl benzene}

\section{Animals}

Sixteen female albino guinea pigs and $16 \mathrm{Wag} / \mathrm{Rij}$ rats (both species obtained from Charles River, Germany) were used. Animals of both species had an average weight of $200 \mathrm{~g}$ at the beginning of the experiment. They were group-housed in wiremesh cages. Food and water were available ad libitum. Lights were on from 7:00 a.m. to 7:00 p.m. Animals were at random assigned to either a control or a treatment group ( $n=8$ per group). The animals' weight was recorded weekly. Possible differences in weight gain between the ethyl benzene-treated animals and control animals were tested with t-tests. Care and use of the animals reported on in experiment 1 were approved by the Animal Care and Use Committee of the Faculty of Medicine, Utrecht University, under number DEC-GNK-95011.

\section{Ethyl benzene exposure}

The animals were exposed to either ambient air or ethyl benzene vapors (guinea pigs $2500 \mathrm{ppm}$; rats $550 \mathrm{ppm}$ ). The rats were exposed for 8 hours/day, for 5 
consecutive days. The guinea pigs, however, were only exposed for $8 \mathrm{~h}$ at the first day. At day two to five the exposure duration was decreased to 6 hours/day, in view of general health problems after the exposure on the first day. During exposure, the animals were placed in wire-mesh cages in a stainless steel Hazleton type chamber. The exposure started at 8:30 a.m. Ethyl benzene (Merck, Schuchardt, 99\% pure) was evaporated at an elevated temperature and the saturated gas was injected into the air supply system, which operated at $20 \mathrm{~m}^{3} / \mathrm{h}$. A dual gas monitor (Brüel \& Kjær, Copenhagen, Denmark, Type 3426) continuously monitored the gas concentration in the chamber. The concentration was kept within $10 \%$ of the desired concentration.

\section{Electrocochleography}

The electrocochleographic techniques were described elsewhere for guinea pigs (Stengs et al., 1997) and are summarized here. These techniques did not have to be changed for rats. Four to eight weeks after the last exposure day, the animals were anaesthetised and both the right and left cochlea were exposed through a ventrolateral approach. Auditory evoked responses were recorded differentially with a silver ball electrode at the apex of the cochlea. A reference electrode was placed in the muscles of the neck. Trains of $8 \mathrm{~ms}$ tone bursts of $1,2,4,8,12,16$ and $24 \mathrm{kHz}$ were presented, with cosine-shaped rise and fall time of $1 \mathrm{~ms}(2 \mathrm{~ms}$ at $1 \mathrm{kHz} ; 1.5 \mathrm{~ms}$ at 2 $\mathrm{kHz}$ ). The averaged responses were saved for off-line analysis. CAP amplitude was defined as the difference between the highest negative peak and the summating potential in the complex electrophysiological response. In general, cochlear potentials evoked by the same sound level were about 10 times higher in guinea pigs than those in rats. Consequently, CAP thresholds were defined differently in both species. As the objective threshold, we used an iso-response criterion at a $10 \mu \mathrm{V}$ level for the guinea pig and at $1 \mu \mathrm{V}$ for the rat. The threshold shift for each exposed animal at each frequency was calculated in reference to the average threshold of the control group at that frequency. Threshold shifts as a consequence of exposure were statistically evaluated by analysis of variance (ANOVA), with species as a between-subjects factor and frequency as a within-subject factor. Post-hoc, differences between rats and guinea pigs per frequency were tested using Tukey's HSD test. Further, to evaluate differences in CAPs at supra-threshold levels, we also used ANOVA (per species), with ethyl benzene exposure as a between-subject factor and frequency and level of 
stimulation as within-subject factors. CAP vs level growth curves were logarithmically transformed before analysis to improve homogeneity of variance and to convert proportional effects into linear ones. Subsequently, data from left and right ears were averaged per animal before ANOVA.

\section{Histology}

Immediately after electrocochleography, the cochleas of both guinea pigs and rats were fixed by transcardial perfusion with a chilled tri-aldehyde fixative consisting of 3\% glutaraldehyde, $2 \%$ formaldehyde, $1 \%$ acrolein, $2.5 \%$ dimethylsulfoxide in 0.1 M sodium cacodylate buffer ( $\mathrm{pH}$ 7.4), followed by overnight immersion in the same fixative at $4^{\circ} \mathrm{C}$. The cochleas were processed further according to the routine method used for guinea pigs (De Groot et al., 1987). Semithin $(1 \mu \mathrm{m})$ mid-modiolar sections were cut and stained with 1\% methylene blue, $1 \%$ azur II in $1 \%$ sodium tetraborate. Sections were examined under the light microscope. In mid-modiolar sections of the cochlea, the organ of Corti was reliably visualized at five locations in the rat and at 8 locations in the guinea pig. Each location was separated from the next one by a halfturn distance. Hair cell counts were performed at each of these locations in both the left and the right cochlea, using 5-6 subsequent mid-modiolar sections. If a hair cell is not seen in these 5-6 subsequent $1 \mu \mathrm{m}$ sections, it can be reliably assumed that the cell is not present. OHC loss was expressed as the percentage of remaining OHCs per location, relative to the expected number of OHCs. All OHC counts were performed by two investigators, independently of one another and unaware of the experimental conditions. Because the mid-modiolar sections provided OHC counts at reproducible locations of the organ of Corti, it was possible to estimate the characteristic frequency at these locations. In guinea pigs we used the place-frequency map of Greenwood (1990), in rats we used the frequency map presented by Müller (1991).

When relevant, ANOVA was used for statistical evaluation of the OHC counts. Ethyl benzene exposure was a between-subjects factor, cochlear location a within-subjects factor. Post-hoc, differences between cochlear locations were tested using Tukey's HSD test. 


\section{Experiment 2: Blood concentrations of ethyl benzene}

\section{Animals and ethyl benzene exposure}

After finding considerable differences in susceptibility to ethyl benzene in experiment 1 , we performed a limited study into the background of this difference. Using similar methods as in experiment 1, four additional rats and four additional guinea pigs were exposed to $500 \mathrm{ppm}$ ethyl benzene for 3 consecutive days, for 8 hours/day. Care and use of the animals reported on in experiment 2 were approved by the Animal Care and Use Committee of TNO Food and Nutrition (Zeist) under no. 602 .

\section{Determination of ethyl benzene blood concentration}

Immediately after the end of the exposure at day one and day three, blood samples $(1 \mathrm{ml})$ were collected by orbital punction (under transient ether anaesthesia). The blood was mixed with heparin $(5000 \mathrm{IE} / \mathrm{ml})$. Ethyl benzene concentration in rat blood and guinea pig blood was determined by gas chromatography. Blood ( $0.5 \mathrm{ml})$ was extracted with $1 \mathrm{ml}$ ethanol and $2 \mathrm{ml} \mathrm{n}$-hexane. As an internal standard, $\mathrm{n}$ dodecane was used. After extraction, $1 \mathrm{ml}$ water was added in order to establish phase separation. Subsequently, the organic layer was pipetted into a glass test tube and reduced under nitrogen to a volume of approximately 50 to $150 \mu \mathrm{l}$. A gas chromatograph (Carlo Erba Instruments, Milano, Italy, Model 5360) equipped with a split/splitless injector and flame ionisation detector was used. One $\mu l$ of each sample was injected with an autosampler using splitless injection. A capillary column (CP-Sil$5-\mathrm{CB}, 50 \mathrm{~m} \times 0.32 \mathrm{~mm}$ (ID), film thickness $1.2 \mu \mathrm{m}$ ) was used. The column was operated initially at $45^{\circ} \mathrm{C}$ for $1 \mathrm{~min}$, after which the temperature was increased at $5^{\circ} \mathrm{C} / \mathrm{min}$ to $180^{\circ} \mathrm{C}$, followed by $20^{\circ} \mathrm{C} / \mathrm{min}$ to $250^{\circ} \mathrm{C}$. The injection port temperature was $250^{\circ} \mathrm{C}$ and the detector base temperature was $280^{\circ} \mathrm{C}$. The carrier gas was helium with a column inlet pressure of $120 \mathrm{kPa}$. Data acquisition was performed using Chromnet software (Spectra-Physics, San Jose, California). The calibration curves made in hexane were linear from 200 up to $2000 \mathrm{ng} / 2 \mathrm{ml}$. All chemicals used were obtained from Merck (Darmstadt, Germany). The purity of n-dodecane and ethanol was at least $99 \%$. The purity of $n$-hexane and ethyl benzene was $97 \%$ and $99 \%$ respectively as reported by the supplier. The averaged recovery of ethyl benzene in the rat and the guinea pig, for concentration levels in the range from ca 198 up to ca 7928 
$\mathrm{ng} / 0.5 \mathrm{ml}$ blood, was $77 \%$ and $80 \%$, respectively. Reported concentrations for ethyl benzene were adjusted to $100 \%$ recovery. The limit of quantitation was ca $60 \mathrm{ng} / 0.5$ $\mathrm{ml}$ for both rat and guinea pig blood after correction for recovery.

\section{RESULTS}

\section{Experiment 1: Ototoxicity of ethyl benzene}

\section{General health}

At the end of the first day ( 8 hours) of exposure to 2500 ppm ethyl benzene, two out of 8 guinea pigs showed serious signs of ill health. These guinea pigs were motionless and did not respond to painful stimuli. One of them died, seemingly as a result of the exposure. The other animals appeared vertiginous. After shortening the exposure duration from 8 to 6 hours/day, the remaining guinea pigs no longer exhibited overt signs of ill health. The weight of the ethyl benzene-exposed guinea pigs was not different from the weight of the control animals at the end of the exposure period ( $\mathrm{t}$-test, $\mathrm{P}=0.32$ ). Also, the weight of the rats exposed to $550 \mathrm{ppm}$ ethyl benzene did not differ from that of the control rats ( $t$-test, $\mathrm{P}=0.11$ ).

\section{Electrocochleography}

Fig. 1 shows the threshold shift per frequency in the ethyl benzene-exposed groups with respect to the average threshold in the control groups (the $0 \mathrm{~dB}$ level in Fig. 1). Evidently, the guinea pigs' thresholds were not affected by exposure to 2500 ppm ethyl benzene. In contrast, the rats showed large threshold shifts in the midfrequency range after exposure to $550 \mathrm{ppm}$. ANOVA over the entire data set, rats and guinea pigs included, showed main effects of species $\left(F_{(1,13)}=27.8 ; P<0.01\right)$ and frequency $\left(F_{(6,78)}=17.4 ; P<0.01\right)$, and an interaction between both $\left(F_{(6,78)}=12.2\right.$; $\mathrm{P}<0.01)$. Subsequently, post hoc Tukey HSD confirmed a difference in threshold shift between rats and guinea pigs at $8,12,16$ and $24 \mathrm{kHz}$ (all $\mathrm{P}<0.01$ ).

Supra-threshold effects of ethyl benzene on the CAP at $16 \mathrm{kHz}$ are shown in Fig. 2. As mentioned before, CAP amplitude at the same stimulus level differs considerably between rats and guinea pigs. Clearly, there was no effect of ethyl benzene on the $16 \mathrm{kHz}$ CAP growth curves in the guinea pig. ANOVA, performed on this growth curve and on those evoked by all other tested frequencies, confirmed this. 


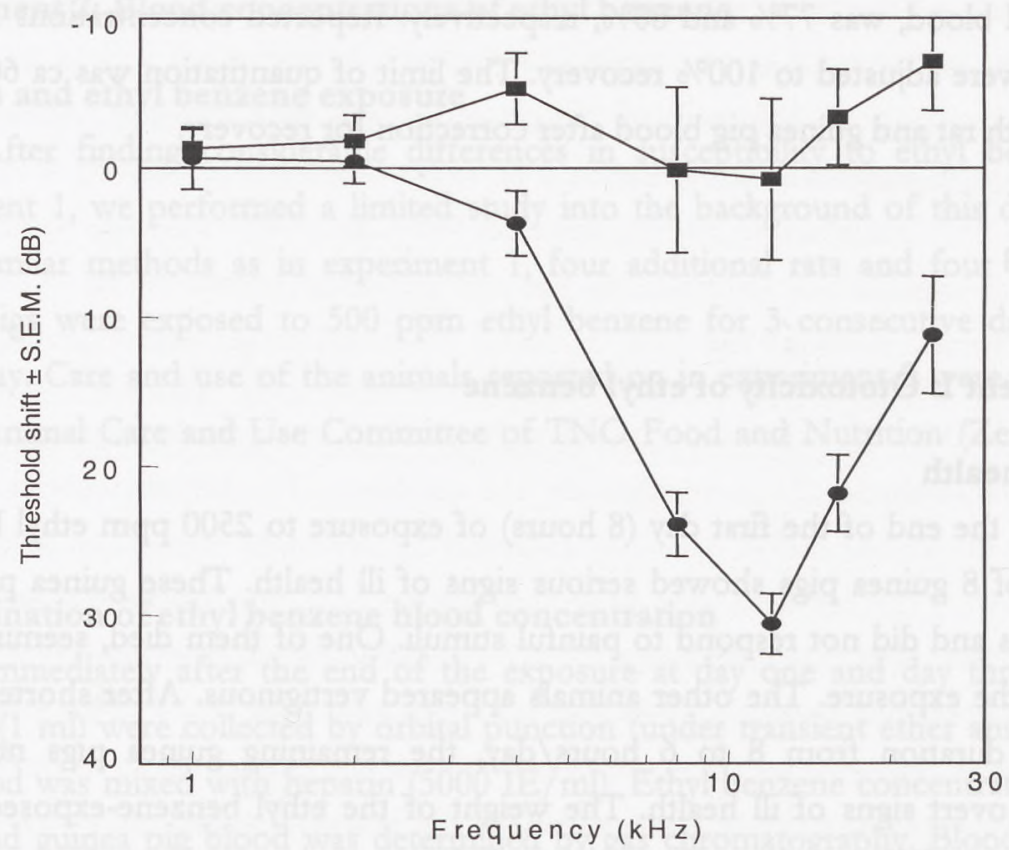

Figure 1. Ethyl benzene exposure (550 ppm, 8 hours/day, 5 days) resulted in a midfrequency hearing loss in rats (- $\bullet$-). In guinea pigs (- - ), the thresholds were not affected by ethyl benzene at a much higher exposure level (2500 ppm, 6 hours/day, 5 days). Thresholds were based on CAP iso-response levels at $1 \mu \mathrm{V}$ in the rats and at $10 \mu \mathrm{V}$ in the guinea pigs. Threshold shifts were calculated in reference to the average thresholds in the non-exposed control groups.

However, clear effects were found in the $\mathrm{CAP}$ growth curves at $16 \mathrm{kHz}$ in rats: The curve for the ethyl benzene exposed animals is shifted to higher stimulus levels. ANOVA, performed on the rat data only, with all tested frequencies included, showed significant main effects of ethyl benzene $\left(\mathrm{F}_{(1.13)}=54.0 ; \mathrm{P}<0.01\right)$, frequency $\left(\mathrm{F}_{(6.78)}=133.2 ; \mathrm{P}<0.01\right)$ and level of stimulation $\left(\mathrm{F}_{(8.104)}=743.6 ; \mathrm{P}<0.01\right)$. Interactions were found between all main effects. The interaction between treatment and level of stimulation reflects the steeper growth of the CAP amplitude with level in the ethyl benzene exposed animals and is reminiscent of the psychophysical phenomenon "loudness recruitment". The interaction between ethyl benzene and frequency was further pursued with ANOVA at each stimulus frequency separately. The effect of ethyl benzene on the CAP growth curves was significant at $8 \mathrm{kHz}\left(\mathrm{F}_{(1,13)}=49.8\right.$; $\mathrm{P}<0.01), 12 \mathrm{kHz}\left(\mathrm{F}_{(1,13)}=49.7 ; \mathrm{P}<0.01\right), 16 \mathrm{kHz}\left(\mathrm{F}_{(1,13)}=14.6 ; \mathrm{P}<0.01\right)$ and $24 \mathrm{kHz}$ $\left(\mathrm{F}_{(1,13)}=8.8 ; \mathrm{P}<0.05\right)$. 

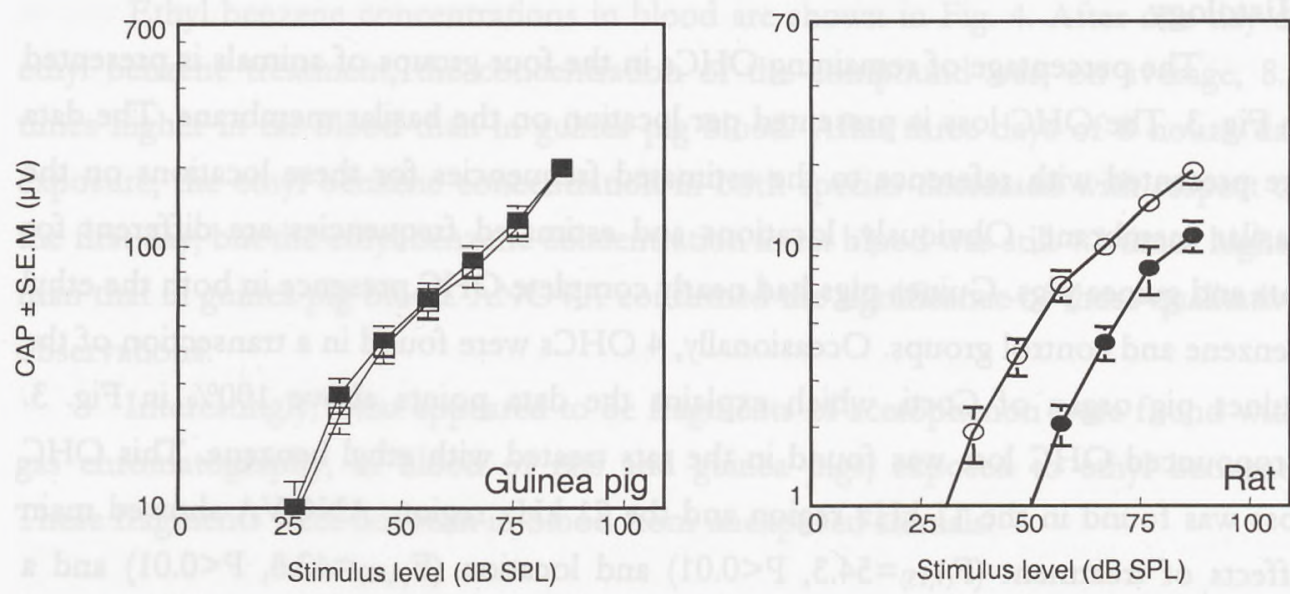

Figure 2. Effects of ethyl benzene on CAP growth curves at $16 \mathrm{kHz}$ for guinea pigs (- $\square-$ $=$ control group $(n=8)$; - $-=2500$ ppm ethyl benzene 6 hours/day, 5 days $(n=7))$ and rats $(-0-=$ control group $(n=7)$; - $\bullet=2500$ ppm ethyl benzene 6 hours/day, 5 days $(n=8))$.

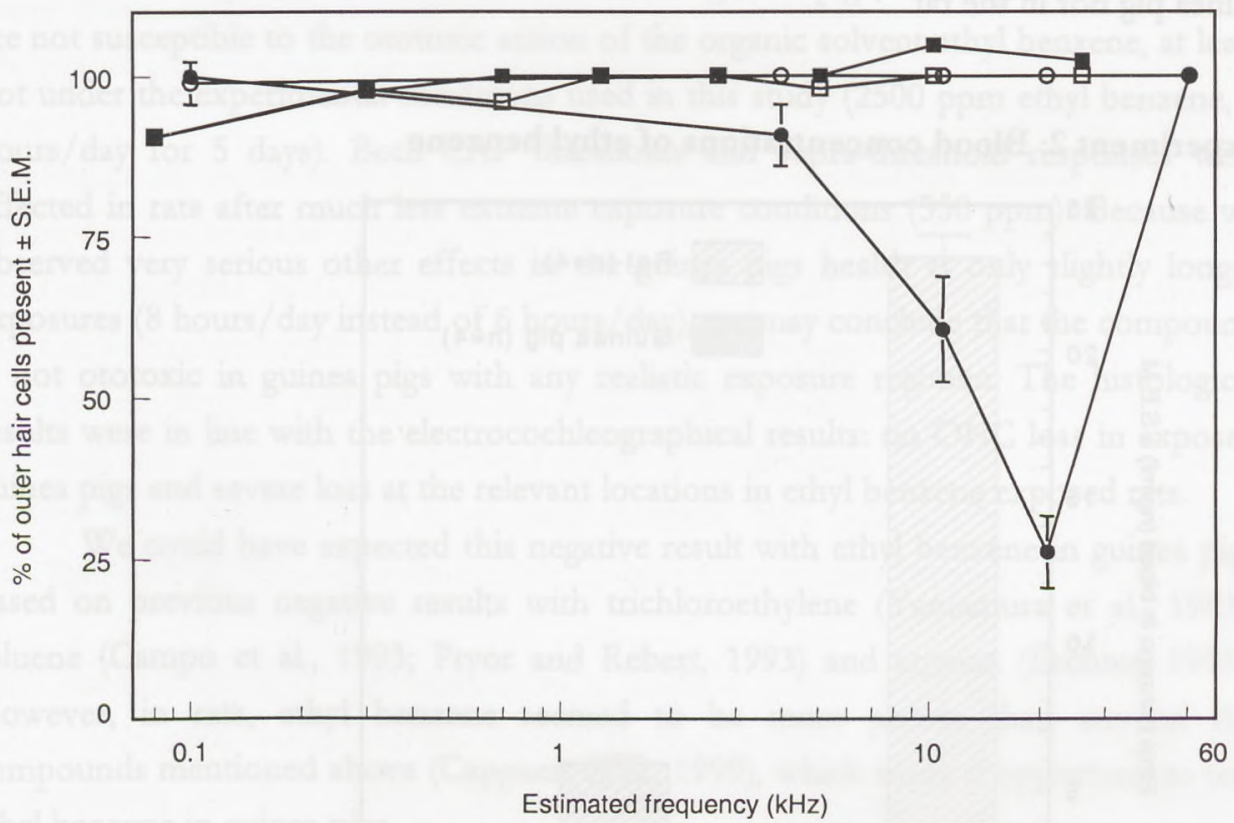

Figure 3. Percentage of remaining outer hair cells (OHCs) for guinea pigs (- $\square-:$ control group; -n-: exposed group) and rats (-O-: control groupl - -:: exposed group). The horizontal axis gives the estimated characteristic frequencies of the locations where the hair cells were counted. 


\section{Histology}

The percentage of remaining OHCs in the four groups of animals is presented in Fig. 3. The OHC loss is presented per location on the basilar membrane. The data are presented with reference to the estimated frequencies for these locations on the basilar membrane. Obviously, locations and estimated frequencies are different for rats and guinea pigs. Guinea pigs had nearly complete $\mathrm{OHC}$ presence in both the ethyl benzene and control groups. Occasionally, $4 \mathrm{OHCs}$ were found in a transection of the guinea pig organ of Corti, which explains the data points above $100 \%$ in Fig. 3. Pronounced OHC loss was found in the rats treated with ethyl benzene. This OHC loss was found in the $11 \mathrm{kHz}$ region and the $21 \mathrm{kHz}$ region. ANOVA showed main effects of treatment $\left(\mathrm{F}_{(1,13)}=54.3, \mathrm{P}<0.01\right)$ and location $\left(\mathrm{F}_{(4,52)}=42.8, \mathrm{P}<0.01\right)$ and a significant interaction between the two $\left(\mathrm{F}_{(4,52)}=46.6, \mathrm{P}<0.01\right)$. Post hoc analysis (Tukey HSD) on treatment per location showed that the OHC loss in the 11 and $21 \mathrm{kHz}$ region was significant $(\mathrm{P}<0.01)$. Inner hair cell loss was never seen, neither in the guinea pig nor in the rat.

\section{Experiment 2: Blood concentrations of ethyl benzene}

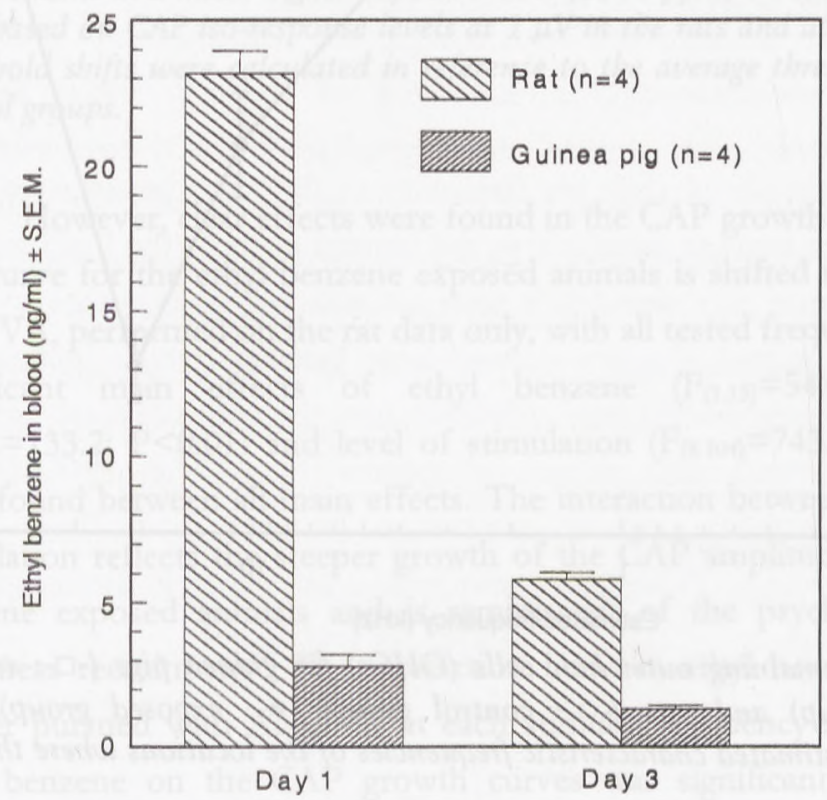

Figure 4. Average concentration of ethyl benzene in rat and guinea pig blood, after one and three days of ethyl benzene exposure (500 ppm, 8 hours/day). 
Ethyl benzene concentrations in blood are shown in Fig. 4. After one day of ethyl benzene treatment, the concentration of the compound was, on average, 8.3 times higher in rat blood than in guinea pig blood. After three days of 8 hours/day exposure, the ethyl benzene concentration in both species decreased with respect to the first day, but the ethyl benzene concentration in rat blood was still 4.3 times higher than that in guinea pig blood. ANOVA confirmed the significance of these qualitative observations.

Interestingly, what appeared to be fragments of acetophenon were found with gas chromatography, in blood of rats and guinea pigs, exposed to ethyl benzene. These fragments were not seen in blood from unexposed animals.

\section{DISCUSSION}

This study has shown that mature albino guinea pigs, in sharp contrast to rats, are not susceptible to the ototoxic action of the organic solvent ethyl benzene, at least not under the experimental conditions used in this study (2500 ppm ethyl benzene, 6 hours/day for 5 days). Both CAP thresholds and supra-threshold responses were affected in rats after much less extreme exposure conditions (550 ppm). Because we observed very serious other effects in the guinea pigs health at only slightly longer exposures (8 hours/day instead of 6 hours/day), we may conclude that the compound is not ototoxic in guinea pigs with any realistic exposure regimen. The histological results were in line with the electrocochleographical results: no OHC loss in exposed guinea pigs and severe loss at the relevant locations in ethyl benzene exposed rats.

We could have expected this negative result with ethyl benzene in guinea pigs based on previous negative results with trichloroethylene (Yamamura et al., 1983), toluene (Campo et al., 1993; Pryor and Rebert, 1993) and styrene (Fechter, 1993). However, in rats, ethyl benzene seemed to be more potent than any of the compounds mentioned above (Cappaert et al., 1999), which made it opportune to test ethyl benzene in guinea pigs.

Another rodent species, mice, was susceptible to toluene ( $\mathrm{Li}$ et al., 1992). $\mathrm{C} 57 \mathrm{BL} / 6 \mathrm{~J}$ mice were exposed to $1000 \mathrm{ppm}$ toluene at 1 or 6 months of age. The mice showed a mild hearing loss at $31.5 \mathrm{kHz}$ or $16 \mathrm{kHz}$ which depended on the age at the time of exposure. Chinchillas showed a mild loss which was confined to the $16 \mathrm{kHz}$ 
region after exposure to $2000 \mathrm{ppm}$ toluene during 12 hours/day for 10 days (Davis et al., 1996). Rats, exposed to the same exposure regimen but only 8 hours/day had hearing losses of 20 and $18 \mathrm{~dB}$ at 16 and $32 \mathrm{kHz}$, respectively (Davis et al., 1996). In summary, different rodent species have different susceptibility to the ototoxic effects of organic solvents. Although not all solvents have been tested in all species, the general pattern seems to be that rats are the most susceptible species, guinea pigs the least, and chinchillas and mice take intermediate positions.

In principle, the insusceptibility in guinea pigs could be due to inherent insusceptibility of the auditory system or to a toxicokinetic or metabolic mechanism that prevents the solvents from reaching the auditory periphery. An argument against inherent insusceptibility of the auditory system in guinea pigs is provided by Liu et al. (1997). They reported that isolated OHCs from the guinea pig cochlea showed a marked shortening in response to superfusion with $100 \mu \mathrm{M}$ toluene. Concomitantly, the intracellular $\mathrm{Ca}^{2+}$ concentration increased (Liu and Fechter, 1997), which might be connected with OHC loss observed in vivo. Thus, the second hypothesis seems more plausible to explain the insusceptibility found in guinea pigs: the solvents simply do not reach their target.

Our second experiment was designed to test this hypothesis. In agreement with the hypothesis it showed a marked difference in ethyl benzene concentration between rat and guinea pig blood after identical exposures. Thus, although we only determined ethyl benzene concentrations in blood at one exposure concentration (500 $\mathrm{ppm})$, the simplest explanation for the difference in susceptibility between the species is that in guinea pigs the concentration of the compound in the inner ear does not reach toxic concentrations. This suggests that there is a difference in uptake, distribution, metabolism and/or excretion of the solvents between guinea pigs and rats.

The purpose of an animal model is to extrapolate the results to humans. The sensitivity of the human auditory system to organic solvents is not quite clear at the moment, but there is growing evidence that the human auditory periphery is susceptible to organic solvents. Solvent abusers, who are exposed to high concentration of solvents, showed hearing loss and abnormal ABR recordings (Lazar et al., 1983; Metrick et al., 1982). Hearing loss has also been reported in occupationally exposed individuals (Muijser et al., 1988; Möller et al., 1990; Morata et al., 1994). Moreover, a highly increased relative risk (11 times) for hearing loss was found in 
workers exposed to both organic solvents and noise (Morata et al., 1993). Evidently, an important issue is the applicability of rats and guinea pigs as models for human solvent ototoxicity. Given the fact that humans seem susceptible and guinea pigs are definitely not susceptible, rats appear to be the best animal model to study different aspects of solvent ototoxicity as it relates to humans.

\section{ACKNOWLEDGEMENTS}

The authors wish to thank R. Bulder, J.C.M.J de Groot and F. Hendriksen for their histological assistance and E. Duistermaat for his technical assistance with the ethyl benzene exposures. This study was supported by grants of NWO-MW (grant number 9004-63-085) and by the Heinsius-Houbolt Fund. 


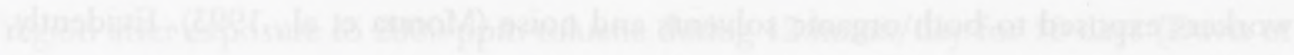

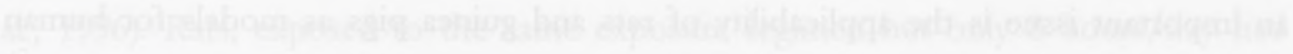

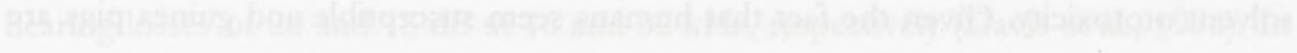

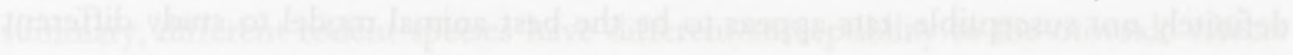

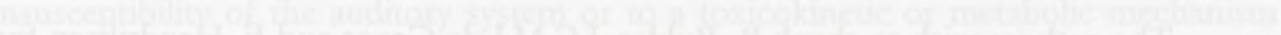
-

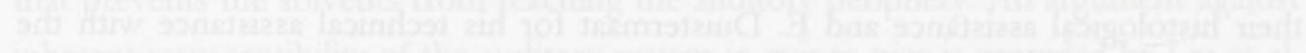

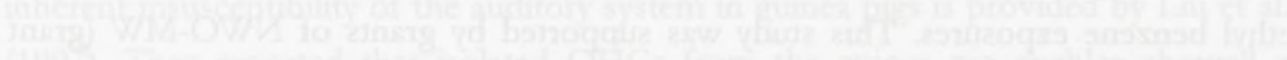

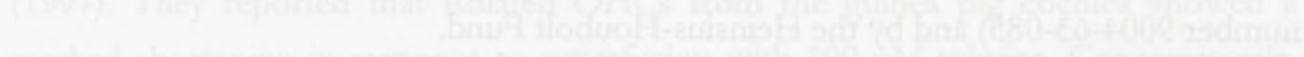
rans 


\section{THE THIRD ROW OF OUTER HAIR CELLS HARDLY CONTRIBUTES TO COCHLEAR SENSITIVITY}

The present study, a re-analysis of a subset of the data presented in Chapter 5 shows the relative unimportance of the third row of OHCs with regard to cochlear sensitivity in the rat. The relation between histological damage in the form of hair cell counts and physiological hearing loss in the form of shifts in iso-response level was evaluated in rats after exposure to either $300 \mathrm{ppm}$ ethyl benzene or $400 \mathrm{ppm}$ ethyl benzene or 105 $\mathrm{dB}$ noise ( 8 hours/day for 5 days). After ethyl benzene exposure, severe loss of thirdrow $\mathrm{OHCs}$ without accompanying hearing loss is observed. On the other hand, noise exposure results in a distinct hearing loss without accompanying loss in the third row, but with minor loss in the first row. Thus, it seems that the first row of OHCs is more important for normal hearing than the third row of OHCs.

(Co-authors: Klis, Smoorenburg) 


\section{INTRODUCTION}

Outer hair cells (OHCs) in the mammalian cochlea are required for normal hearing sensitivity. When all OHCs are absent, a hearing loss of 40-50 dB can occur (e.g., Kiang et al., 1976; Dallos and Harris, 1978; Liberman, 1987). In all mammals except the monotremes (egg-laying mammals) the OHCs are arranged in three rows: the first or inner row, the second or middle row and the third or outer row of OHCs (called $\mathrm{OHC1}, \mathrm{OHC} 2$ and $\mathrm{OHC}$, respectively). Little is known about the relative contribution of each row to the sensitivity of the cochlea. Exposure to noise or ototoxic agents such as aminoglycoside, antibiotics and cisplatin, causes hearing loss and preferentially damages the first row of OHCs. Thus, by inference, it has been proposed that the first row contributes most to cochlear sensitivity (Liberman and Dodds, 1984). Yoshida and Liberman (1999) provided further evidence for the importance of the first row with respect to cochlear sensitivity in guinea pigs showing anomalous orientation of the stereociliary bundles in the first row OHCs.

Organic solvents are ototoxic agents that can affect the cochlea both in its function and morphology (e.g., Cappaert et al., 1999). Two striking differences as compared to most other ototoxic agents have emerged from the literature: 1) The effects of organic solvents are localised in the mid-frequency region (e.g. Crofton et al., 1994), whereas ototoxic agents like cisplatin and aminoglycosides induce auditory impairment predominantly in the high-frequency region; 2) OHC damage caused by organic solvents is most prominent in the third row and the loss decreases dramatically towards the other rows (Johnson and Canlon, 1994; Campo et al., 1997; Loquet et al., 1999). Thus, solvent-induced ototoxicity can be used as a complementary model to study the relative contributions of the three rows of OHCs to cochlear sensitivity.

The purpose of this paper is to quantitatively assess the contribution of especially the third $\mathrm{OHC}$ row to cochlear sensitivity in rats exposed to either the organic solvent ethyl benzene or to noise. We compared the OHC loss per row with electrocochleographically recorded hearing loss in the same ear. 


\section{MATERIALS AND METHODS}

The data in this paper were gathered in a study designed to investigate the effects of simultaneous exposure to noise and ethyl benzene on the auditory system. The complete study is presented in Chapter 5, where the methods are described. Briefly, male Wag/Rij rats (obtained from Charles River, Germany) were used, with an average weight of $200 \mathrm{~g}$ at the start of the experiment. Groups of animals were exposed to either $300 \mathrm{ppm}$ ethyl benzene or $400 \mathrm{ppm}$ ethyl benzene or $105 \mathrm{~dB}$ SPL broad-band noise. The rats were exposed for 8 hours/day for 5 consecutive days. Per group, we randomly chose 5 animals of the original 8 and used the right ears for the analyses presented here.

Electrocochleography and harvesting of the cochleas took place between 3 to 7 weeks after the exposure. During these procedures, the rats were anaesthetised with a pre-operative intramuscular injection of Thalamonal $(0.1 \mathrm{ml} / 100 \mathrm{~g}$ body weight; Thalamonal contains $2.5 \mathrm{mg} / \mathrm{ml}$ Droperidol and $0.05 \mathrm{mg} / \mathrm{ml}$ Fentanyl) followed by artificial ventilation with a gas mixture containing $33 \% \quad \mathrm{O}_{2}, 66 \% \mathrm{~N}_{2} \mathrm{O}$ and $1 \%$ Halothane through a trachea cannula. Heart frequency was monitored and body temperature was kept at $38^{\circ} \mathrm{C}$. Care and use of the animals reported on in this paper were approved by the Animal Care and Use Committee of the Faculty of Medicine, University Medical Centre Utrecht, under number DEC-GNK-95011.

Electrocochleography was performed with a silver ball electrode at the apex of the cochlea. The cochlear potential of interest in this paper is the compound action potential (CAP). Trains of $8 \mathrm{~ms}$ tone bursts of $1,2,4,8,12,16$ and $24 \mathrm{kHz}$ were presented, with cosine-shaped rise and fall times of $1 \mathrm{~ms}$, except at $1 \mathrm{kHz}(2 \mathrm{~ms})$ and at $2 \mathrm{kHz}(1.5 \mathrm{~ms})$. The inter-stimulus interval was $99 \mathrm{~ms}$. The responses were amplified and band-pass filtered $(-12 \mathrm{~dB} /$ octave, $-3 \mathrm{~dB}$ at $1 \mathrm{~Hz}$ and $10 \mathrm{kHz})$. The averaged responses were saved for off-line analysis. The CAP amplitude was defined as the difference between the first negative peak and the summating potential in the electrophysiologic response.

Immediately after electrocochleography, the cochleas were removed from the animal and fixed and processed further according to the routine method used for guinea pigs (De Groot et al., 1987). Then, surface preparations were made according to the block-surface method of Spoendlin and Brun (1974) with minor alterations (Chapter 5). With this method, the presence of a hair cell is judged entirely by the 
presence of its stereociliary bundle. The number of inner hair cells (IHCs) and OHCs per row was determined and compiled in cytocochleograms. These cytocochleograms were constructed with estimates of the frequencies along the basilar membrane based on the place-frequency map of Müller (1991).

In the electrocochleographic recordings, we acquired full amplitude growth curves at each frequency. For clarity and to be able to compare these data directly with the histological data, consisting of one data-point per frequency, we needed a method to characterize the amplitude growth curves with a single attribute. In this paper, a CAP iso-response level at $1 \mu \mathrm{V}$ was chosen. The average stimulus levels needed to evoke this CAP amplitude in a previously measured control group were $38,37,24,15$, 26,14 and $22 \mathrm{~dB}$ SPL for $1,2,4,8,12,16$ and $24 \mathrm{kHz}$, respectively. These levels are close to behavioural thresholds (Fay, 1988). Shifts in exposed groups were calculated with respect to the data recorded in the control group.

\section{RESULTS}

All relevant data are presented in Fig. 1. In this figure a comparison is made between hair cell loss per row, depicted in the left panels and accompanying effects on hearing, based on shifts in CAP iso-response levels, in the right panels.

In the group exposed to $300 \mathrm{ppm}$ ethyl benzene, considerable loss was detected in the third row of OHCs, amounting to approximately $15 \%$ in the region where it peaked. Nevertheless, there was no sign of hearing loss based on CAP isoresponse levels in that region. The latter observation was confirmed by ANOVA. The entire curve describing the CAP iso-response levels as a function of frequency was not significantly different from the control curves.

The discrepancy between loss of third-row OHCs and electrophysiologically evaluated loss was even more pronounced after $400 \mathrm{ppm}$ ethyl benzene. Maximum loss of third-row OHCs amounted to $80 \%$, but still there was no electrophysiological sign of hearing loss. Once again, these observations were confirmed by ANOVA. It is important to note that besides severe loss of third-row OHCs, mild to moderate OHC losses were found in the first and second row, respectively. Apparently, these losses are not important enough to evoke hearing loss either. 

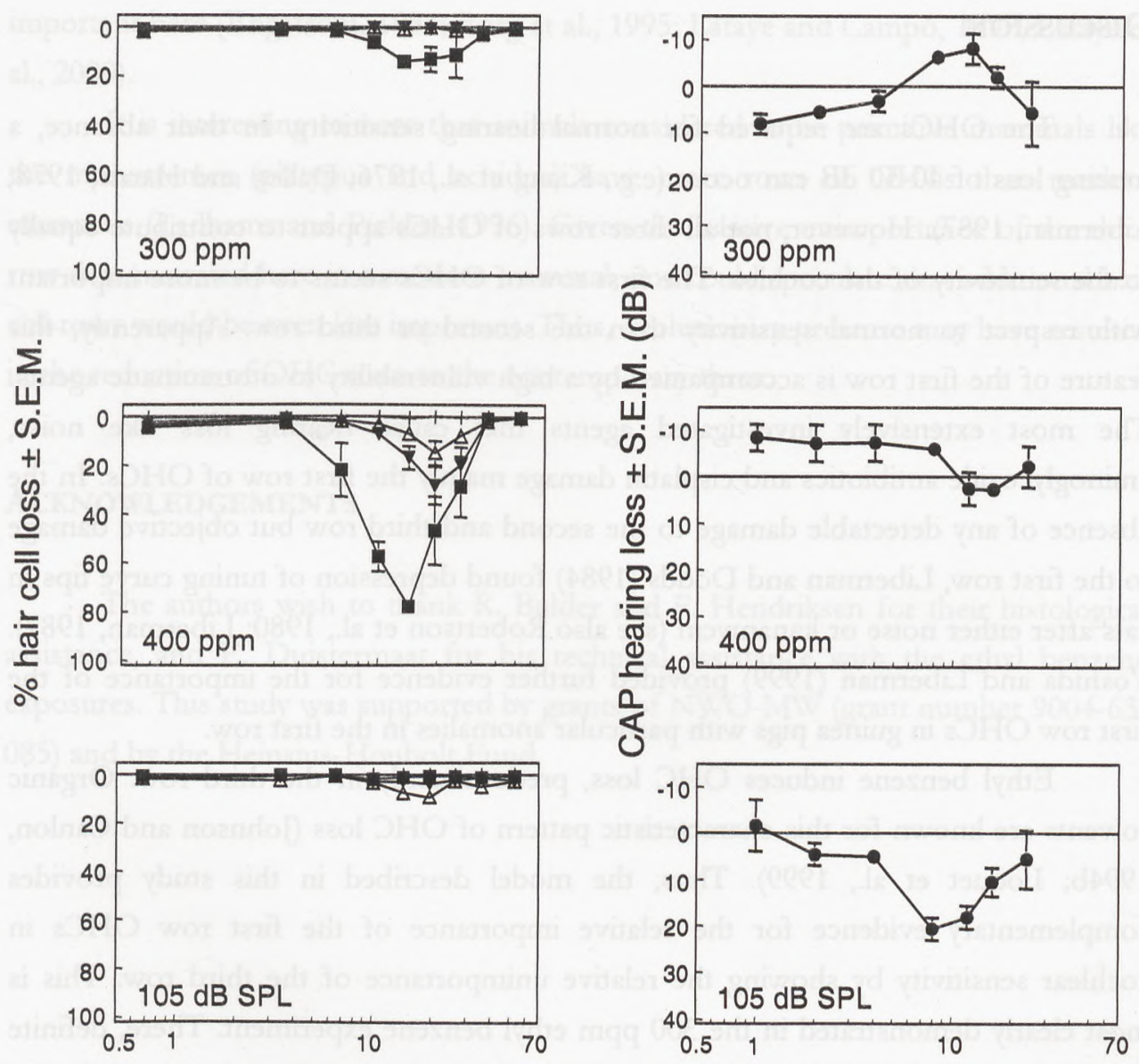

Estimated frequency $(\mathrm{kHz})$

Figure 1. The average CAP hearing loss \pm S.E.M. ( $n=5$ ears) as a function of stimulus frequency is presented in the right panels and the average hair cell loss per row as a function of estimated frequency is shown in the left panels after exposure to 300 ppm ethyl benzene, 400 ppm ethyl benzene and $105 \mathrm{~dB}$ SPL noise. $+=$ IHC; $\Delta=$ first row of $\mathrm{OHCs} ; \boldsymbol{\nabla}=$ second row of $\mathrm{OHCs} ; \mathbf{m}=$ third row of $\mathrm{OHCs}$.

The situation after noise exposure can be described as a mirror image of the situation after ethyl benzene exposure. After $105 \mathrm{~dB}$ SPL broad-band noise exposure, we found clear loss in the CAP data (ANOVA: P<0.05). However, the cytocochleograms reveals only minor loss, which is limited to the first row. In contrast to the situation after ethyl benzene, no loss was seen in the third row of OHCs. 


\section{DISCUSSION}

The OHCs are required for normal hearing sensitivity. In their absence, a hearing loss of 40-50 dB can occur (e.g., Kiang et al., 1976; Dallos and Harris, 1978; Liberman, 1987). However, not all three rows of OHCs appear to contribute equally to the sensitivity of the cochlea. The first row of OHCs seems to be more important with respect to normal sensitivity than the second or third row. Apparently, this feature of the first row is accompanied by a high vulnerability to ototraumatic agents. The most extensively investigated agents that cause hearing loss like noise, aminoglycoside antibiotics and cisplatin damage mainly the first row of OHCs. In the absence of any detectable damage to the second and third row but objective damage to the first row, Liberman and Dodds (1984) found depression of tuning curve tips in cats after either noise or kanamycin (see also Robertson et al., 1980; Liberman, 1987). Yoshida and Liberman (1999) provided further evidence for the importance of the first row OHCs in guinea pigs with particular anomalies in the first row.

Ethyl benzene induces OHC loss, predominantly in the third row. Organic solvents are known for this characteristic pattern of OHC loss (Johnson and Canlon, 1994b; Loquet et al., 1999). Thus, the model described in this study provides complementary evidence for the relative importance of the first row OHCs in cochlear sensitivity by showing the relative unimportance of the third row. This is most clearly demonstrated in the $300 \mathrm{ppm}$ ethyl benzene experiment. There, definite OHC loss was seen in the third row whereas the other two rows were completely unaffected. However there was no sign of accompanying hearing loss. The result after $400 \mathrm{ppm}$ is more complicated because here the other two rows are also affected. Nevertheless, the OHC loss in the third row is predominant and it is still not accompanied by relevant hearing loss. Exposure to $105 \mathrm{~dB}$ SPL noise resulted in significant hearing loss. Yet, there was only minor loss in the first row, definitely not in the third. It is unlikely that this minor loss in the first row accounts for the hearing loss observed. The effects on the first row after $400 \mathrm{ppm}$ ethyl benzene seem to be larger than the effects on that row after noise exposure whereas the reverse applies to the hearing loss. The simplest explanation for this discrepancy is that functional damage after noise exposure may occur without complete loss of the OHCs. Subcellular damage, not detectable with the techniques used in this study, might be 
important here (Engström, 1984; Borg et al., 1995; Lataye and Campo, 1997; Lataye et al., 2000).

It is interesting to note that animals considered to be primitive mammals like the monotremes (platypus and echidna) have more rows of OHCs than modern mammals (Ladhams and Pickles, 1996). Given the relative unimportance of the third row demonstrated here in a modern mammal, one could speculate that additional hair cell rows would be even less important. Thus, evolutionary pressure may have resulted in the reduction of $\mathrm{OHC}$ rows to the contemporary three.

\section{ACKNOWLEDGEMENTS}

The authors wish to thank R. Bulder and F. Hendriksen for their histological assistance, and E. Duistermaat for his technical assistance with the ethyl benzene exposures. This study was supported by grants of NWO-MW (grant number 9004-63085) and by the Heinsius-Houbolt Fund. 


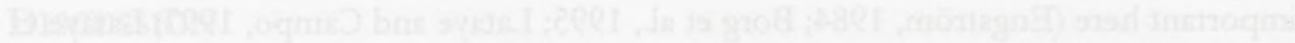

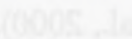

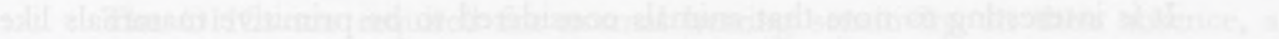

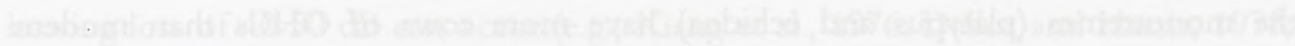

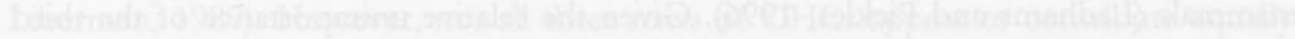

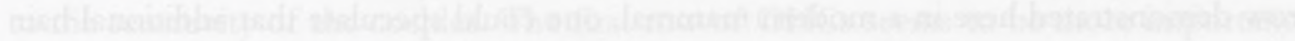

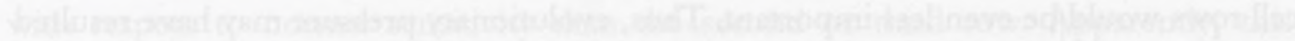

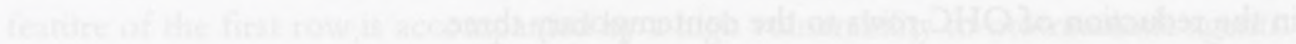




\section{ADDENDUM}

PURE TONE VERSUS AUDITORY EVOKED POTENTIAL THRESHOLDS IN COCHLEAR HEARING LOSS: MANIFESTATIONS OF DEGRADING TEMPORAL INTEGRATION

(Accepted by Physiology and Psychophysical Bases of Auditory Function; co-authors: Schoonhoven, van Zanten) 


\section{INTRODUCTION}

Because the auditory system is capable of integrating incoming sound energy over short time intervals, signal detection thresholds depend on the duration of the acoustic stimulus. Thresholds decrease with stimulus duration by about $10 \mathrm{~dB}$ per decade up to durations of $200-300 \mathrm{~ms}$, beyond which thresholds are constant. The capability of temporal integration is reduced in cochlear hearing loss (Florentine et al., 1988), and as a consequence the amount of hearing loss measured depends on the duration of the stimulus applied. Behavioural tests using conventional pure tone audiometry are based on stimulus durations in the order of a second, well beyond the integration time window. In conditions where behavioural testing is not feasible, such as in clinical testing of small children or in experimental studies involving hearing impaired animals, thresholds are usually determined with auditory evoked potentials such as the VIIIth nerve compound action potential (CAP) or the auditory brainstem response $(A B R)$. Due to the transient nature of these responses, the observed thresholds are related to the onset time of the stimulus, typically in the millisecond range. Therefore, the issue of temporal integration becomes relevant in comparing behavioural and evoked potential thresholds (Gorga et al., 1984; Gorga and Thornton, 1989; Conijn et al., 1992a). In an earlier report (Schoonhoven et al., 1996) we have elaborated on this point with respect to pure tone and VIIIth nerve CAP threshold data in human adults, showing that there is a significant difference in growth of the two types of thresholds as a function of cochlear deterioration. The goal of the present study is to extend this analysis to other comparisons of behavioural versus electrophysiological threshold data, including human infant and adult ABR studies and VIIIth nerve CAP data in experimental animals.

\section{MODEL}

The concept of temporal integration in normal and impaired hearing is schematised in Figure 1. The (increased) threshold measured in a pathological ear is indicated by $T_{1}$ for a long stimulus duration as e.g. used in pure tone audiometry, and by $T_{s}$ for a short stimulus duration in the millisecond range. The difference between $T_{1}$ and $T_{s}$, denoted by $\Delta T$, is a function of hearing loss, i.e. of $T_{l}$, and of course of the 
stimulus durations associated with $T_{1}$ and $T_{s}$. When we assume that $\Delta T$ can be approximated as a linear function of hearing loss over the range of interest with constant of proportionality $\beta$

$$
\Delta \mathrm{T}=\mathrm{T}_{1}-\mathrm{T}_{\mathrm{s}}=\beta \mathrm{T}_{1}
$$

we have

$$
\mathrm{T}_{\mathrm{s}}=(1-\beta) \mathrm{T}_{1}
$$

Therefore, the model predicts $T_{s}$ to be linearly related to $T_{l}$ with a slope less than one. The same model was elaborated in a slightly different notation in Schoonhoven et al. (1996) to which we refer for further details.

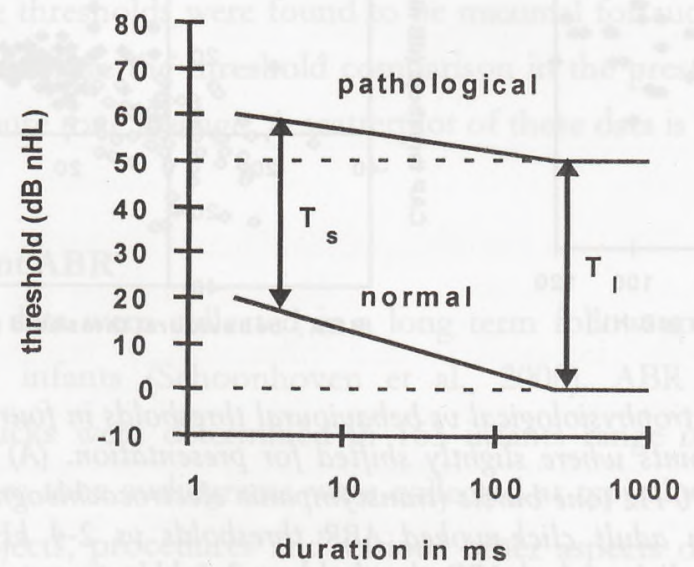

Figure 1. Schematised representation of temporal integration in normal and impaired hearing. The threshold $T$ of a pathological ear relative to normal is smaller for short $\left(T_{s}\right)$ than for long $\left(T_{l}\right)$ duration stimuli. The difference $\Delta T=T_{l}-T_{s}$ increases with the hearing loss, as conventionally measured by $T_{l}$.

\section{EXPERIMENTAL DATA}

This paper includes four different sets of data regarding electrophysiological and behavioural response thresholds: human adult VIIIth nerve CAPs, infant ABR and adult $\mathrm{ABR}$, all compared with conventional pure tone audiometry, and rat VIIIth nerve CAPs compared with thresholds determined with reflex modification audiometry (RMA). 

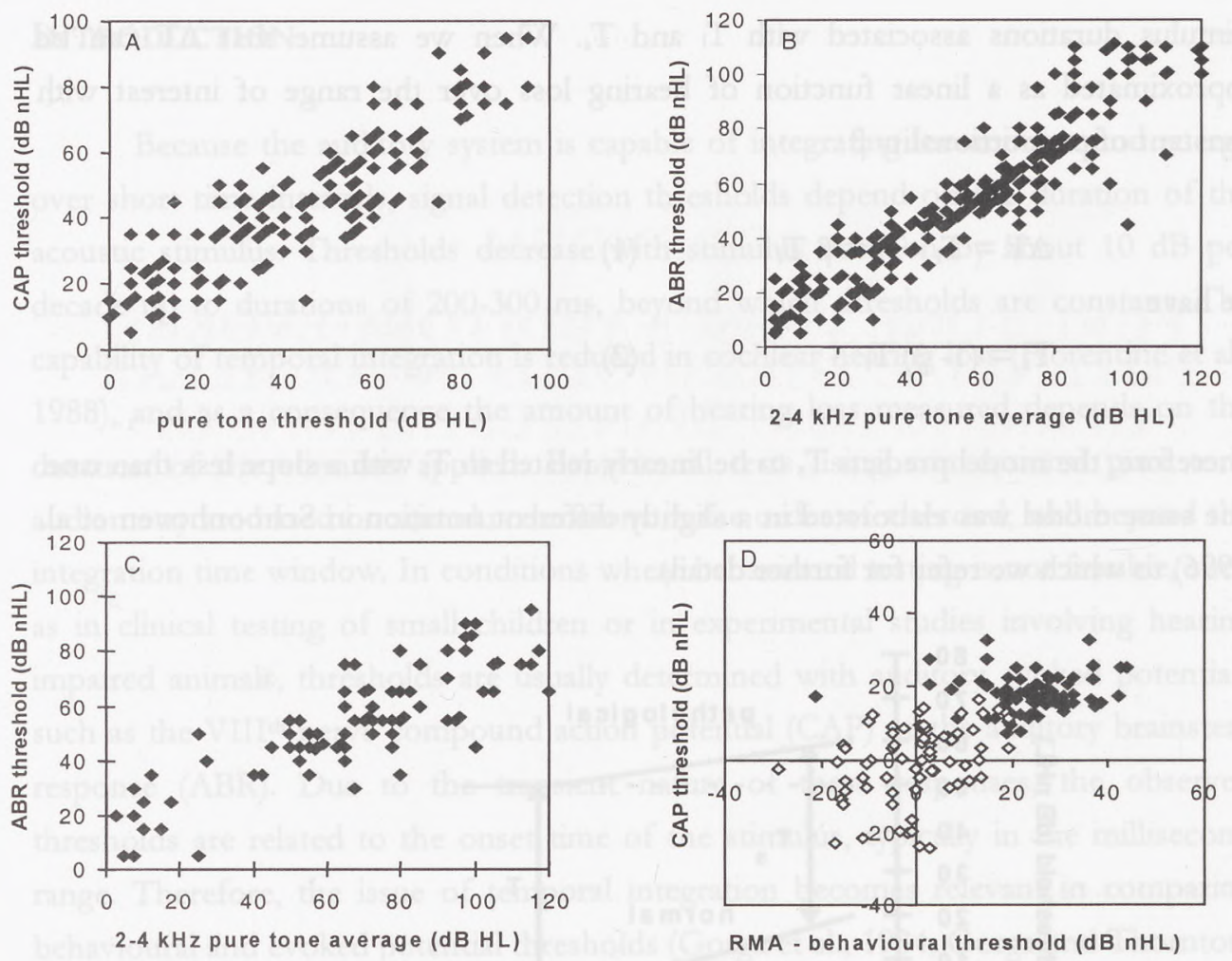

Figure 2. Scatterplots of electrophysiological vs behavioural thresholds in four different sets of data. Coinciding data points where slightly shifted for presentation. (A) human VIII ${ }^{\text {th }}$ nerve CAP thresholds to $2000 \mathrm{~Hz}$ tone bursts (transtympanic electrocochleography) vs pure tone thresholds, (B) human adult click-evoked ABR thresholds vs 2-4 kHz pure tone average, (C) human infant click-evoked $A B R$ threshold vs $2-4 \mathrm{kHz}$ pure tone average in follow-up audiometry, (D) rat VIII ${ }^{\text {th }}$ nerve CAP vs reflex thresholds in normal animals (open symbols) and animals exposed to ethyl benzene (closed symbols); data pooled over all frequencies.

\section{Human adult VIII th nerve CAPs}

These data are recapitulated here from an earlier study (Schoonhoven et al., 1996) to which we refer for further details. Briefly, CAP response thresholds $(0.1 \mathrm{mV}$ amplitude criterion) were determined to tone burst stimuli (octave frequencies from 500 to $8000 \mathrm{~Hz}, 4 \mathrm{~ms}$ plateau, 2 periods rise/fall time) in 148 adult human ears with a variety of type and degree of cochlear hearing loss, as part of a routine clinical transtympanic electrocochleography procedure. Comparisons were made with pure 
tone thresholds at the same frequencies. A scatterplot of the $2000 \mathrm{~Hz}$ CAP thresholds vs pure tone threshold at the same frequency is shown in Figure 2A.

\section{Human adult ABR}

In a study of the relation between $A B R$ thresholds and pure tone audiograms (Van der Drift et al., 1987), ABRs were recorded to $0.1 \mathrm{msec}$ rectangular clicks (re normal behavioural thresholds to the same clicks) in 209 adult human ears with various degrees of cochlear hearing loss. ABR thresholds were compared with pure tone thresholds at each audiometric frequency and at several pure tone averages. Details of experimental procedures and various correlation and regression results have been published by Van der Drift et al. (1987). Correlations between click-evoked ABR and pure tone thresholds were found to be maximal for audiometric frequencies of 2 and $4 \mathrm{kHz}$; therefore the threshold comparison in the present study concentrates on the $2-4 \mathrm{kHz}$ pure tone average. A scatterplot of these data is shown in Figure 2B.

\section{Human infant ABR}

These data were collected in a long term follow-up study of ABR threshold estimation in infants (Schoonhoven et al., 2000). ABR thresholds to $0.1 \mathrm{msec}$ rectangular clicks were determined in 163 infants range of typically 1-3 years old. Follow-up pure tone audiograms were collected at typically 4-8 years of age. Details regarding subjects, procedures and various other aspects of the data were given by Schoonhoven et al. (2000). Many infants had hearing losses so large that no ABR could be elicited even at the highest stimulus level available. Several ears were excluded from the analysis because of the existence of middle ear infection during $\mathrm{ABR}$ testing. Reliable and complete $\mathrm{ABR}$ and audiometric thresholds were available from 83 ears. The analysis given here again concentrates on the comparison of clickevoked ABR thresholds with the $2-4 \mathrm{kHz}$ pure tone average. A scatterplot of these data is shown in Figure 2C.

\section{Rat VIII'th nerve CAP}

These data were collected in a project investigating possible ototoxic effects of various organic solvents (Cappaert et al., 1999). Wag/Rij rats were exposed by 
inhalation to either air $(n=16)$ or $800 \mathrm{ppm}$ ethyl benzene ( $\mathrm{n}=16), 8$ hours/day for 5 days. Reflex modification audiometry (RMA) was performed four weeks after the last exposure. RMA estimates the auditory thresholds by recording the reduction of a noise evoked startle response when it is preceded by a single-frequency sound stimulus, the prepulse (Jaspers et al., 1993). The startle-eliciting stimulus was a noise burst of $40 \mathrm{~ms}$ at $110 \mathrm{~dB}$ SPL. The prepulse was a $40 \mathrm{~ms}$ tone burst of $4,8,12,16$ or $24 \mathrm{kHz}, 100 \mathrm{~ms}$ before the noise burst. The auditory thresholds for all tested frequencies were obtained by fitting the startle responses as a function of prepulse level to a previously described model (Crofton, 1992; Jaspers et al., 1993). The same animals were subjected to electrocochleography to estimate the CAP thresholds $(1 \mu \mathrm{V}$ amplitude criterion). Stimuli were $8 \mathrm{~ms}$ tone bursts with $1 \mathrm{~ms}$ rise/fall time. Further details can be found in Cappaert et al. (1999). A scatterplot of the data, pooled over all stimulus frequencies, is shown in Figure 2D.

\section{Data analysis}

Statistical analysis of the data included correlation and regression analysis. The method of orthogonal regression analysis was applied to estimate the fundamental underlying relation between behavioural and physiological thresholds. Unlike conventional linear regression, which assumes the independent variable to be precisely known, and assigns all experimental uncertainty to the dependent variable, orthogonal regression yields an estimate of the linear relation between quantities $\xi$ and $\eta$, measured through experimental observations $\mathrm{x}$ and $\mathrm{y}$ which are both contaminated with experimental errors: $x_{i}=\xi_{i}+e_{i}$ and $y_{i}=\eta_{i}+f_{i}$, respectively. The resulting regression equation between $\xi$ and $\eta$ is unique in that it does not depend on whether orthogonal regression of $\mathrm{y}$ on $\mathrm{x}$ or of $\mathrm{x}$ on $\mathrm{y}$ is taken; the regression line does not suffer the 'flattening' effect of conventional linear regression lines in case of imperfect correlation of the data. For further conceptual and mathematical details regarding orthogonal regression we refer to Schoonhoven et al. (1996).

\section{RESULTS}

Results of correlation and orthogonal regression of physiological on behavioural thresholds for the four sets of data are given in Table 1. Note that for all 
human $\mathrm{CAP}$ and $\mathrm{ABR}$ data the physiological thresholds are compared with pure tone thresholds, related to stimulus duration in the order of one second, while for the animal data behavioural thresholds were determined for stimulus durations $40 \mathrm{~ms}$. The correlation in the latter data is clearly weaker than that in the three human studies. The important parameter in Table 1 in the present context is the slope of the orthogonal regression line, and the error therein. Based on the construction of a $95 \%$ confidence interval, the slope of the relation between transient evoked potential threshold and the behavioural thresholds is significantly smaller than one in all four sets of data. The parameter $\beta$ defined in Eq. 1 can be calculated as one minus the slope of the regression line. Its value varies between 0.17 and 0.33 for the different experiments.

Table 1. Results of correlation analysis and of orthogonal regression analysis of electrophysiological on behavioural thresholds, applied to the four sets of data shown in Figure 2, and the resulting estimate of $\beta$ (Eq. 1).

\begin{tabular}{lcccccc}
\hline & $\begin{array}{c}\text { correlation } \\
\text { coefficient }\end{array}$ & $\begin{array}{c}\text { slope } \\
(\mathrm{dB} / \mathrm{dB})\end{array}$ & $\begin{array}{c}\text { SD slope } \\
(\mathrm{dB} / \mathrm{dB})\end{array}$ & $\begin{array}{c}\text { offset } \\
(\mathrm{dB})\end{array}$ & $\begin{array}{c}\text { SD offset } \\
(\mathrm{dB})\end{array}$ & $\begin{array}{c}\beta \text { (Eq 1) } \\
(\mathrm{dB} / \mathrm{dB})\end{array}$ \\
\hline human CAP & 0.87 & 0.83 & 0.04 & 8.4 & 1.8 & 0.17 \\
adult ABR & 0.93 & 0.90 & 0.03 & 3.90 & 1.6 & 0.10 \\
infant ABR & 0.85 & 0.67 & 0.05 & 7.5 & 3.3 & 0.33 \\
rat CAP & 0.69 & 0.68 & 0.06 & 1.1 & 1.0 & 0.32 \\
\hline
\end{tabular}

\section{Discussion}

Although degradation of temporal integration in human hearing impaired subjects has been documented in several different ways the mechanism of this aspect of hearing impairment has not been clarified unequivocally. Combined psychophysical and neurophysiological experiments in the same subjects may provide valuable additional information on that point. For the present human CAP data we have shown before (Schoonhoven et al., 1996) that differences in growth rate of physiological and pure tone thresholds $(\beta=0.83 \pm 0.04$ in Table 1$)$ match remarkably well with estimates of the parameter $\beta$ from literature data of conventional pure tone thresholds and behavioural thresholds to stimuli as brief as the tone-pip onset times relevant for generation of the CAP $(\beta=0.86 \pm 0.04$, derived from Florentine et al., 
1988). Therefore, we are confident that the results of the present analysis are in essence equally valid for the other types of data presented here. The strength of the present approach might be increased by including the behavioural thresholds to the same brief stimuli used in the evoked potential experiments, which however were not available in this retrospective study. One caveat must be made regarding the human ABR data where the comparison was made between the physiological threshold to a broadband click and the (frequency specific) pure tone threshold. There is general agreement that correlation of the $A B R$ is maximal with the pure tone thresholds in the $2-4 \mathrm{kHz}$ region. However, it cannot be excluded that the observed effect is caused in part by an increasingly sloping character of the more severely hearing impaired subjects' audiograms, making that the dominant contribution to the ABR shifts from the $2-4 \mathrm{kHz}$ to e.g. the $1-2 \mathrm{kHz}$ or even lower frequency range. The results in this context depend on the distribution of different types of hearing losses (e.g. lowfrequency, high-frequency or flat) in the test population (e.g. Conijn et al., 1992b).

The present data give strong evidence of degradation of temporal integration in hearing impaired animals. Notably, the difference in slope from one is one of the largest (and thus $\beta$ one of the largest) in the animal data, where in fact the smallest effect was anticipated because behavioural thresholds were determined to $40 \mathrm{~ms}$ tonebursts and the difference in stimulus durations was therefore an order of magnitude smaller than in the other data sets. However, quantitative comparison with the present human data should be made with care because the RMA threshold is based on reflex tests and may therefore not be directly comparable with pure tone thresholds in humans.

Table 2. Prediction of behavioural from auditory evoked potential thresholds. Results of conventional linear regression analysis applied to the four sets of data shown in Figure 2.

\begin{tabular}{lccccc}
\hline & $\begin{array}{c}\text { slope } \\
(\mathrm{dB} / \mathrm{dB})\end{array}$ & $\begin{array}{c}\text { SD slope } \\
(\mathrm{dB} / \mathrm{dB})\end{array}$ & $\begin{array}{c}\text { offset } \\
(\mathrm{dB})\end{array}$ & $\begin{array}{c}\text { SD offset } \\
(\mathrm{dB})\end{array}$ & $\begin{array}{c}\text { error in the } \\
\text { estimate }(\mathrm{dB})\end{array}$ \\
\hline human CAP & 1.03 & 0.05 & 2.0 & 2.3 & 11.9 \\
adult ABR & 1.01 & 0.03 & 1.3 & 1.8 & 11.6 \\
infant ABR & 1.19 & 0.08 & 3.8 & 4.6 & 16.7 \\
rat CAP & 0.91 & 0.08 & 3.9 & 1.2 & 11.2 \\
\hline
\end{tabular}


With values of $\beta$ in the order of 0.3 , auditory evoked potential thresholds increase at a rate of only $70 \%$ of the pure tone threshold. This conclusion concerns the fundamental growth rate of the two different types of thresholds. The main application of evoked potential studies deals with the prediction of behavioural from evoked potential response thresholds. Irrespective of the analysis presented here, for the purpose of prediction conventional linear regression analysis is the appropriate method, with the evoked potential threshold the independent variable and the behavioural threshold the dependent variable. Table 2 summarizes results of conventional linear regression for the present four sets of data. Note that in spite of the flattening of the regression lines expected because correlation is less than one, slopes for the human data are larger than one. Thus, predicted behavioural thresholds may truly be higher than the evoked potential threshold from which they are estimated (cf Schoonhoven et al., 2000). Similar conclusions may hold with respect to the prediction of true (i.e. not reflex-based) behavioural thresholds in animals, which may have relevant implications for studies using animal models of hearing loss in humans. 


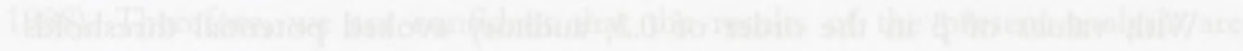

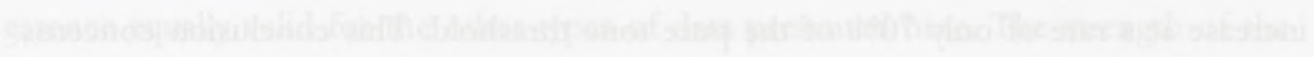

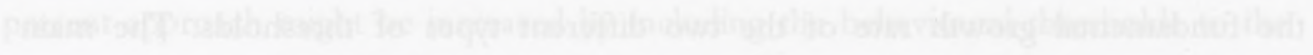

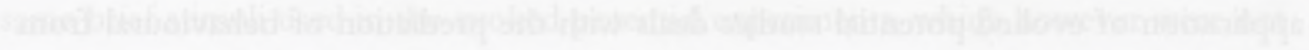

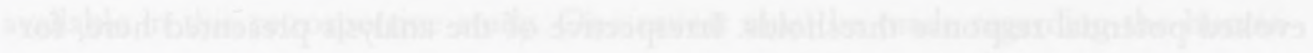

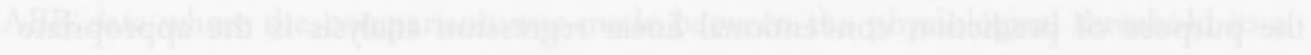

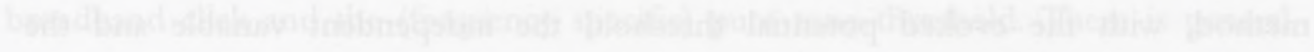

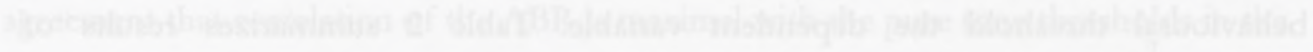

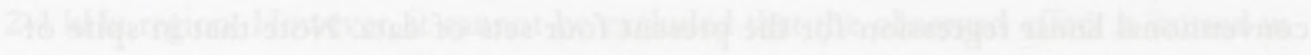

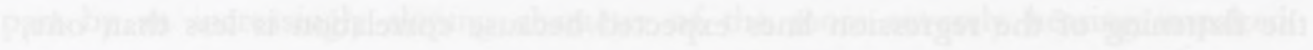

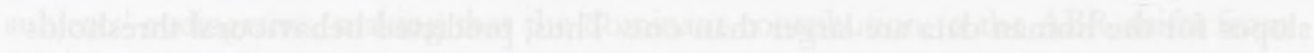

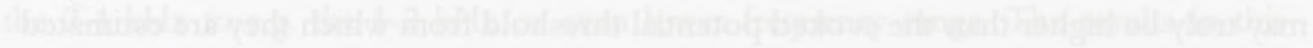

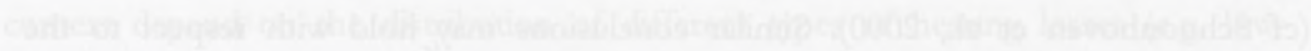

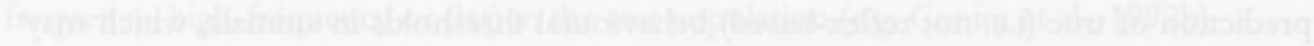

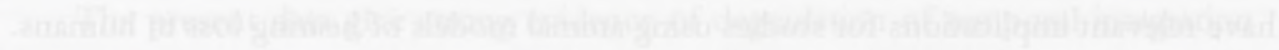




\section{SUMMARY \& CONCLUSIONS}

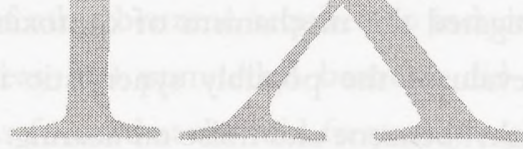


Hearing loss as a result of exposure to excessive noise is a well-known problem in industrialized countries. A less understood and less-known problem is hearing loss induced by exposure to various organic solvents, e.g., toluene, trichloroethylene and styrene. There is increasing evidence that combined exposure to noise and organic solvents may induce a more pronounced hearing loss than would be predicted from exposure to noise or solvents alone. In this thesis, we developed an animal model for characterizing the ototoxic potential of one particular organic solvent, ethyl benzene. Concurrently, we investigated the mechanism of ototoxic action of this solvent. In addition, we tried to evaluate the possibly synergistic interaction associated with combined exposure to ethyl benzene and noise on hearing.

The introduction, Chapter 1, provides a short overview on the workings of the peripheral auditory system. Additionally, the experimental methods used in this thesis (reflex modification audiometry, electrocochleography, measurement of distortion product otoacoustic emissions and histology) are briefly discussed. Finally, an overview of the literature is given concerning the effects of noise, organic solvents, and the combination of these two agents, on hearing.

Chapter 2 focuses on the ototoxicity of ethyl benzene. Rats were exposed to ethyl benzene at $800 \mathrm{ppm}$ for 8 hours/day for 5 consecutive days. Ethyl benzene exposure induced a hearing loss of approximately $20 \mathrm{~dB}$ for all frequencies tested (4$24 \mathrm{kHz}$ ). This was assessed with behavioural reflex modification audiometry (RMA) and measurement of compound action potentials (CAPs). Subsequently, outer hair cell (OHC) counts were performed on midmodiolar sections of the cochleas to quantify the $\mathrm{OHC}$ loss. OHC loss was confined to the mid-frequency region of the cochlea $(11-21 \mathrm{kHz})$ and amounted to approximately $60 \%$ at its peak.

OHC loss and reduced CAPs point at a predominantly peripheral ototoxic effect of ethyl benzene in rats. The OHCs, especially those in the mid-frequency region, are the primary target for this compound. Compared to other organic solvents, ethyl benzene seems to be a very potent ototoxic agent. Toluene, styrene and trichloroethylene have to be applied at much higher concentrations and for longer periods of time to induce effects comparable to those observed in this study. 
The main purpose of the dose-response study presented in Chapter 3 was to further explore the concentration range at which ethyl benzene is ototoxic in rats, using the $800 \mathrm{ppm}$ dose applied in Chapter 2 as a starting point. Rats were exposed to $0,300,400$ or $550 \mathrm{ppm}$ ethyl benzene for 8 hours/day for 5 consecutive days. Distortion product otoacoustic emissions (DPOAEs) and CAP measurements were used to evaluate the hearing loss physiologically. Histological examination was performed on midmodiolar sections of the cochleas to assess the OHC loss. The minimum concentration of ethyl benzene required to induce hearing loss and OHC loss was $400 \mathrm{ppm}$, whereas $300 \mathrm{ppm}$ ethyl benzene did not affect the auditory periphery. The damage was focused at the mid-frequency region of the cochlea. With increasing ethyl benzene level (from $400 \mathrm{ppm}$ to $800 \mathrm{ppm}$, see also Chapter 2) the amount of hearing loss and $\mathrm{OHC}$ loss increased and the frequency range affected broadened.

With this study, a basis for the combination studies was laid and our previous statement on the high ototoxic potency of ethyl benzene was confirmed.

In Chapter 4 the effects of three levels of broad-band noise on hearing in rats are presented. The purpose of this study was to provide a basis for the combination studies and to add to the rather limited information we have on the long-term effects of noise on hearing in this species. Rats were exposed to 90,100 or $110 \mathrm{~dB}$ SPL broad-band noise for 8 hours/day for 5 consecutive days. Thus, the exposure regimen was equal to the one used in the above studies on solvent ototoxicity. Hearing was tested with DPOAE and CAP measurements. Subsequently, OHC counts were performed on midmodiolar sections of the cochleas. Only the highest two noise levels affected hearing. After $100 \mathrm{~dB}$ SPL noise, only the CAPs evoked by $12 \mathrm{kHz}$ stimulation were affected. A more pronounced hearing loss was found after $110 \mathrm{~dB}$ noise in the $4-24 \mathrm{kHz}$ region. Surprisingly, no OHC loss was found in any of the three exposed groups. The latter result may be related to subcellular damage after the noise exposures. These exposures might have injured the stereocilia of the IHCs and the first row of OHCs, not obliterating them.

We compared the results of the present study and results of other experiments in which rats were exposed to noise for more than one day. As a common measure for noise exposition we calculated the total exposure based on the equal-energy theory. This comparison shows a consistent relation, the higher the exposure level, the 
higher the accompanying hearing loss. This relation is consistent, despite the fact that the types of noise, exposure regimens used and methods of evaluating hearing loss were variable in the studies included. Thus, the equal-energy concept provides a useful descriptor of hearing loss in rats exposed to noise.

Chapter 5 evaluates the effects on hearing of simultaneous exposure to ethyl benzene and broad-band noise in the rat. The ethyl benzene concentrations and noise levels applied in this study were based on the dose-effect studies discussed in Chapters 3 and 4 . The concentrations and levels chosen were relatively low, because we wanted to focus on small effects for each agent separately, thereby increasing the chance of detecting synergy when combined. The effects of three ethyl benzene concentrations (0 ppm, $300 \mathrm{ppm}$ or $400 \mathrm{ppm}$ ) and three noise levels (95 or $105 \mathrm{~dB}$ SPL or background noise) and all combinations were examined. For evaluating peripheral damage we used DPOAEs, CAPs and hair cell counts. In addition to the midmodiolar hair cell counts we also evaluated the hair cell loss in this study by inspecting surface preparations of the hair cell arrays using Nomarski differential optics and allows the allocation of missing OHCs to their row of origin.

Ethyl benzene alone, applied at 300 or 400 ppm, did not cause any significant hearing loss, whether assessed by measuring DPOAEs or CAPs. There is a slight discrepancy here with Chapter 3, where we did find some hearing loss after $400 \mathrm{ppm}$ ethyl benzene. However, in line with Chapter 3, pronounced OHC loss was found after 400 ppm ethyl benzene, especially in the third row of OHCs. The second and first row were affected less severely. Noise exposure, in line with Chapter 4, had a profound effect on the physiological data but not on the OHC counts. Both DPOAE and $\mathrm{CAP}$ recordings showed hearing loss after $105 \mathrm{~dB}$ noise applied alone. Only minor OHC loss was detected in the first row of OHCs.

Combined exposure to ethyl benzene ( $300 \mathrm{ppm}$ and $400 \mathrm{ppm}$ ) and $95 \mathrm{~dB}$ noise did not affect the physiological measurements (DPOAEs and CAPs). Increasing the noise level to $105 \mathrm{~dB}$ in combination with either ethyl benzene concentration induced moderate hearing loss. However, this hearing loss did not exceed the hearing loss induced by $105 \mathrm{~dB}$ noise alone. Thus, judging from the physiological measurements, there were no signs of a synergistic interactions. In contrast, synergistic interactions in $\mathrm{OHC}$ loss were detected at multiple locations and in all rows of OHCs, but especially in the most-affected third row. 
The first four studies discussed in this thesis are focused on the rat as an animal model. However, solvent-induced ototoxicity seems to be species dependent. Guinea pigs generally show less or no effects of solvent exposure on hearing. Triggered by the high ototoxicity of ethyl benzene in the rat model, we decided to also evaluate the ototoxic potency of this compound in the guinea pig. The results are presented in Chapter 6. First, in a pilot study, we exposed guinea pigs for 8 hours/day, 5 days/week. In the first week, the concentration applied was $800 \mathrm{ppm}$, in the second week the concentration was increased to $1600 \mathrm{ppm}$, and in the last week the concentration was further increased to $2000 \mathrm{ppm}$. The animals were continually tested for hearing loss with behavioural methods, but nothing was found. In agreement with the behavioural results, electrocochleography (CAPs) did not show any hearing loss. In addition, no OHC loss was seen. In a second experiment, the guinea pigs were exposed to $2500 \mathrm{ppm}$ (for 6 hours/day for 5 days). This for guinea pigs almost lethal dose of ethyl benzene did not induce any damage to the auditory periphery, which was confirmed in both physiological (CAPs) and morphological tests (OHC counts). Based on the results of these two experiments, we may conclude that guinea pigs are far less susceptible to high concentrations of ethyl benzene than rats. In an attempt to explain this difference in susceptibility, we performed a limited study on blood concentrations of ethyl benzene in both species after exposure to $500 \mathrm{ppm}$ ethyl benzene ( 8 hours/day for 3 days). The concentration of ethyl benzene at the end of day 1 in rat blood was 8.3 times the concentration in guinea pig blood. Although the absolute concentrations of ethyl benzene in blood decreased in both species, the concentration was still 4.3 times the concentration in guinea pig blood 3 days after the exposure. Thus, it seems that the difference in susceptibility between both species can be explained by differences in uptake, distribution, metabolism and/or excretion of ethyl benzene.

An important issue, illustrated by the results in this Chapter, is the applicability of animal models for statements or policies about solvent ototoxicity in humans. Given the fact that humans seem susceptible to ototoxic solvents and guinea pigs are not, for the moment rats appear to be the best animal model to study the different aspects of solvent ototoxicity as it relates to humans. 
Chapters 7 and 8 explore certain details of the data from previous Chapters. In Chapter 7 the relation between loss of OHCs and physiological hearing loss is further evaluated based on a subset of the data presented in Chapter 5. The OHC loss per row after 300 ppm ethyl benzene, 400 ppm ethyl benzene and 105 dB noise was examined and compared with the CAP hearing loss in the corresponding ears. It is shown that there can be a huge OHC loss in the third row without any loss in CAP thresholds after ethyl benzene exposure. In contrast, extensive CAP hearing loss was detected after noise exposure with only slight OHC loss in the first row, the second and third row were not affected at all. Thus, it seems that the first row of OHCs is far more important for normal hearing than the third row of OHCs.

Chapter 8, the addendum, is the result of a collaboration with the Universities of Leiden and Rotterdam. The relation between behavioural testing and objective testing with auditory evoked potentials is investigated in this Chapter. The auditory system integrates the incoming sound energy over short time intervals. Because of this feature, the threshold of signal detection (psychophysically) depends on the duration of the sound stimulus. The threshold decreases with stimulus duration by about $10 \mathrm{~dB}$ per decade up to durations of 200-300 ms, beyond which thresholds are constant. As a consequence of a pathological process, degradation of the temporal integration can occur, i.e., the difference in threshold between longer and shorter stimuli would be smaller in pathological ears. Auditory evoked potentials are typically measured with brief tones or clicks and behavioural thresholds with longer stimuli. This might lead to a systematic error when one tries to estimate the clinically important behavioural hearing loss with measurement of auditory evoked potentials. In this Chapter, this issue is explored using four sets of behavioural and evoked potential data, mostly gathered in clinical practice but including the rat data previously presented in Chapter 2. The conclusion is that in all datasets behavioural threshold shifts due to various disorders are truly higher than the evoked potential threshold shifts would predict. This conclusion incorporated our rat data even though reflex data were used for the rat and we lack knowledge about temporal integration in reflex data. 


\section{General conclusions}

I. Compared to other organic solvents, ethyl benzene is a very potent ototoxic chemical in rats.

II. Guinea pigs are dramatically less susceptible to the ototoxic effects of ethyl benzene than rats.

III. Mild ethyl benzene exposure leads to cellular damage in the third row of OHCs with hardly any consequences in terms of threshold.

IV. Mild noise apparently induces light-microscopically invisible sub-cellular damage with clear physiological consequences.

V. A comparison between threshold measurements based on evoked potentials and on behavioural data shows that evoked potential measurements underestimate behavioural thresholds.

VI. Under specific conditions synergistic interactions between noise and ethyl benzene can occur, in particular in OHC counts. 


\section{REFERENCES}


Alberti P.W., 1988. Noise, the most ubiquitous pollutant. Noise \& Health 1, 3-5.

American Chemical Society, 1996. Production by the U.S. chemical industry. Chemical \& Engineering News, Technical report.

Barregård L., Axelsson A., 1984. Is there an ototraumatic interaction between noise and solvents? Scand. Audiol. 13, 151-155.

Biscaldi G.P., Mingardi M., Pollini G., Moglia A., Bossi M.C., 1983. Acute toluene poisoning. Electroneurophysiological and vestibular investigations. Toxicol. Eur. Res. 3, 271-273.

Boettcher F.A., Henderson D., Gratton M.A., Danielson R.W., Byrne C.D., 1987. Synergistic interactions of noise and other ototraumatic agents. Ear \& Hearing 8, 192-212.

Bohne B.A., Gruner M.M., Harding G.W., 1990. Morphological correlates of aging in the chinchilla cochlea. Hear. Res. 48, 79-92.

Borg E., 1982a. Noise-induced hearing loss in rats with renal hypertension. Hear. Res. 8, 93-99.

Borg E., 1982b. Noise-induced hearing loss in normotensive and spontaneously hypertensive rats. Hear. Res. 8, 117-130.

Borg E., Viberg A., 1987. Age-related hair cell loss in spontaneously hypertensive and normotensive rats. Hear. Res. 30, 111-118.

Borg E., Canlon B., Engström B., 1995. Noise-induced hearing loss. Literature review and experiments in rabbits. Morphological and electrophysiological features, exposure parameters and temporal factors, variability and interactions. Scand. Audiol. Suppl. 40, 1-147.

Brownell W.E., Bader C.R., Bertrand D., De Ribaupierre Y, 1985. Evoked mechanical responses of isolated cochlear outer hair cells. Science 227, 194-196.

Campo P., Lataye R., Bonnet P., 1993. No interaction between noise and toluene on cochlea in the guinea pig. Acta Acustica 1, 35-42.

Campo P., Lataye R., Cossec B., Placidi V., 1997. Toluene-induced hearing loss: a mid-frequency location of the cochlear lesions. Neurotoxicol. Teratol. 19, 129-140.

Cappaert N.L.M., Klis S.F.L., Muijser H., de Groot J.C.M.J., Kulig B.M., Smoorenburg G.F., 1999. The ototoxic effects of ethyl benzene in rats. Hear. Res. 137, 91-102.

Coleman J.W., 1976. Hair cell loss as a function of age in the normal cochlea of the guinea pig. Acta Otolaryngol. 82, 33-40

Comis S.D., Pickles J.O., Osborn M.P., Pepper C.B., 1989. Tip-link organization in anomalously oriented hair cells of the guinea pig cochlea. Hear. Res. 40, 205-212.

Conijn E.A.J.G., Brocaar M.P., van Zanten G.A., 1992a. Low-frequency specificity of the auditory brainstem response threshold elicited by clicks masked with $1590-\mathrm{Hz}$ high-pass noise in subjects with sloping cochlear hearing losses. Audiology 31, 272-83.

Conijn E.A.J.G., Brocaar M.P., van Zanten, G.A., 1992b. Comparison between the frequency specificities of auditory brainstem response thresholds to clicks with and without high-pass masking noise. Audiology 31, 284-92.

Crofton K.M., 1992. Reflex modification and the assessment of sensory dysfunction. In: Tilson H., Mittchell C. (Eds.), Neurotoxicology. Raven Press, New York, NY, pp 181-211.

Crofton K.M., Zhao X., 1993. Mid-frequency hearing loss in rats following inhalation exposure to trichloroethylene: Evidence from reflex modification audiometry. Neurotoxicol. Teratol. 15, 413-423. 
Crofton K.M., Lassiter T.L., Rebert C.S., 1994. Solvent-induced ototoxicity in rats: An atypical selective mid-frequency hearing deficit. Hear. Res. 80, 25-30.

Crofton K.M., Zhao X., 1997. The ototoxicity of trichloroethylene: Extrapolation and relevance of high-concentration, short-duration animal exposure data. Fund. Appl. Toxicol. 38, 101-106.

Dallos P., 1972. Cochlear inner and outer hair cells: Functional differences. Science 177, 356-358.

Dallos P., Cheatham M.A., 1976. Production of cochlear potentials by inner and outer hair cells. J. Acoust. Soc. Am. 60, 510-512.

Dallos P., Harris D., 1978. Properties of auditory nerve responses in absence of outer hair cells. J. Neurophysiol. 41, 365-383.

Dallos, P., 1992. The active cochlea. J. Neurosci. 12, 4575-4585.

Davis R.R., Murphy W.J., Zheng X.Y., Henderson D., Morata T., Khan A., 1996. Effects of toluene and noise on the chinchilla auditory system. Abstracts of the 19th Midwinter Meeting of the Association for Research in Otorhinolaryngology (ARO), 1996, St. Petersburg Beach, Fl.

De Groot J.C.M.J., Veldman J.E., Huizing E.H., 1987. An improved fixation method for guinea pig cochlear tissues. Acta Otolaryngol. (Stock) 104, 234-242.

Engström B., 1984. Fusion of stereocilia on inner hair cells in man and in rabbit, rat and guinea pig. Scand. Audiol. 13, 87-92.

Eybalin E., 1993. Neurotransmitters and neuromodulators of the mammalian cochlea. Physiol. Reviews 73, 309-373.

Fay R.R., 1988. Hearing in vertebrates. A psychophysics databook. Heffenan Press Inc, Worcester, MA.

Fechter L.D., 1993. Effects of acute styrene and simultaneous noise exposure on auditory function in the guinea pig. Neurotox. Teratol. 15, 151-155.

Fechter L.D., Liu Y., Herr D.W., Crofton K.M., 1998. Trichloroethylene ototoxicity: Evidence for a cochlear origin. Toxicol. Sci. 42, 28-35.

Fishbein L., 1985. An overview of environmental and toxicological aspects of aromatic hydrocarbons IV. Ethylbenzene. Sci.Total Environ. 44, 269-287.

Florentine M., Fastl H., Buus, S., 1988. Temporal integration in normal hearing, cochlear impairment and impairment simulated by masking. J. Acoust. Soc. Am. 84, 195-203

Fornazzari L., Wilkinson D.A., Kapur B.M., Carlen P.L., 1983. Cerebellar, cortical and functional impairment in toluene abusers. Acta Neurol. Scand. 67, 319-329.

Fridberger A., Ulfendahl M., 1996. Acute mechanical overstimulation of isolated outer hair cells causes changes in intracellular calcium levels without shape changes. Acta Otolaryngol. (Stock) 116, $17-24$.

Gorga M.P., Beauchaine K.A., Reiland J.K., Worthington D.W., Javel E., 1984. The effects of stimulus duration on ABR and behavioral threshold. J. Acoust. Soc. Am. 76, 616-619.

Gorga M.P., Thornton A.R., 1989. The choice of stimuli for ABR measurements. Ear \& Hearing 10, 217-230.

Greenwood D.D., 1990. A cochlear frequency-position function for several species - 29 years later. J. Acoust. Soc. Am. 87, 2592-2605.

Hudspeth A.J., 1989. How the ear's works work. Nature 341, 397-404. 
Jacobsen P., Hein H.O., Suadicani P., Parving A., Gyntelberg F., 1993. Mixed solvent exposure and hearing impairment: an epidemiological study of 3284 men. The Copenhagen male study. Occup. Med. 43, 180-184.

Jaspers R.M.A., Muijser H., Lammers J.H.C., Kulig B.M., 1993. Mid-frequency hearing loss and reduction of acoustic startle responding in rats following trichloroethylene exposure. Neurotoxicol. Teratol. 15, 407-412.

Johnson A.C., Juntunen L., Nylén P., Borg E., Höglund G., 1988. Effect of interaction between noise and toluene on auditory function in the rat. Acta Otolaryngol. (Stockh) 105, 56-63.

Johnson A.C., Nylén P., Borg E., Höglund G., 1990. Sequence of exposure to noise and toluene can determine loss of auditory sensitivity in the rat. Acta Otolaryngol. (Stockh) 109, 34-40.

Johnson A.C., 1992. Auditory sensitivity in rats exposed to toluene and/or acetyl salicylic acid. Neuroreport 3, 1141-1144.

Johnson A.C., Canlon B., 1994a. Toluene exposure affects the functional activity of outer hair cells. Hear. Res. 72, 189-196.

Johnson A.C., Canlon B., 1994b. Progressive hair cell loss induced by toluene exposure. Hear. Res. 75, 201-208.

Keithley E.M., Feldman M.L., 1982. Hair cell counts in an age-related series of rat cochleas. Hear. Res. $8,249-262$.

Kiang N.Y.S., Liberman M.C., Levine R.A., 1976. Auditory-nerve activity in cats exposed to ototoxic drugs and high-intensity sounds. Ann. Otol. Rhinol. Laryngol. 85, 752-768.

Ladhams A., Pickles J.O., 1996. Morphology of the monotreme organ of Corti and macula lagena. J. Comp. Neurol. 336, 335-347.

Lataye R., Campo P., 1997. Combined effects of a simultaneous exposure to noise and toluene on hearing function. Neurotoxicol. Teratol. 19, 373-382.

Lataye R., Campo P., Loquet G., 1999. Toluene ototoxicity in rats: Assessment of the frequency of hearing deficit by electrocochleography. Neurotoxicol. Teratol. 21, 267-276.

Lataye R., Campo P., Loquet G., 2000. Combined effects of noise and styrene exposure on hearing function in the rat. Hear. Res. 139, 86-96.

Lazar R.B., Ho S.U., Melen O., Daghestani A.N., 1983. Multifocal central nervous system damage caused by toluene abuse. Neurology 33, 1337-1340.

Li H.-S., Johnson A.-C., Borg E., Höglund G., 1992. Auditory degeneration after exposure to toluene in two genotypes of mice. Arch. Toxicol. 66, 382-286.

Liberman M.C., Dodds L.W., 1984. Single-neuron labeling and chronic cochlear pathology. III. Stereocilia damage and alterations of threshold tuning curves. Hear. Res. 16, 55-74.

Liberman M.C., 1987. Chronic ultrastructural changes in acoustic trauma: Serial-section reconstruction of stereocilia and cuticular plates. Hear. Res. 26, 65-88.

Liu Y., Fechter L.D., 1997. Toluene disrupts outer hair cell morphometry and intracellular calcium homeostasis in cochlear cells of guinea pigs. Toxicol. Appl. Pharmacol. 142, 270-277.

Liu Y., Rao D., Fechter L.D., 1997. Correspondence between middle frequency auditory loss in vivo and outer hair cell shortening in vitro. Hear. Res. 112, 134-140.

Loquet G., Campo P., Lataye R., 1999. Comparison of toluene-induced and styrene-induced hearing losses. Neurotoxicol. Teratol. 21, 698-697. 
Mäkitie A., 1997. The ototoxic effect of styrene and its interaction with noise. $\mathrm{PhD}$-thesis, University of Helsinki, Finland.

Melchiar I., Syka J., Ulehlová L., 1980. Recovery of the endocochlear potential and the $\mathrm{K}^{+}$ concentrations in the cochlear fluids after acoustic trauma. Hear. Res. 2, 55-63.

Metrick S.A., Brenner R.P., 1982. Abnormal brainstem auditory evoked potentials in chronic paint sniffers. Ann. Neurol. 12, 553-556.

Möller C., Ödkvist L., Larsby B., Tham R., Ledin T., Bergholtz L., 1990. Otoneurological findings in workers exposed to styrene. Scand. J. Work Environ. Health 16, 189-194

Morata T.C., Dunn D.E., Kretschmer L.W., Lemasters G.K., Santos,U.P., 1991. Effects of simultaneous exposure to noise and toluene on workers' hearing and balance. Proceedings of the fourth international Conference on the combined environmental factors, 81-86.

Morata T.C., Dunn D.E., Kretschmer L.W., Lemasters G.K., Keith R.W., 1993. Effects of occupational exposure to organic solvents and noise on hearing. Scand. J. Work. Environ. Health. 19, 245-254.

Morata T.C., Dunn D.E., Sieber W.K. 1994. Occupational exposure to noise and ototoxic organic solvents. Arch. Environ. Health 49, 359-365.

MorataT.C., Dunn D.E., Kretschmer L.W., Lemasters G.K., Santos U.P., 1991. Effects of simultaneous exposure to noise and toluene on workers' hearing and balance. Proceedings of the fourth international Conference on the combined environmental factors, 81-86.

Muijser H., Hoogendijk E.M.G., Hooisma J., 1988. The effects of occupational exposure to styrene on high-frequency hearing thresholds. Toxicology 49, 331-340.

Muijser H., Lammers J.H.C.M., Kulig B.M. 2000. Effects of exposure to trichloroethylene and noise on hearing in rats. Noise \& Heatlh 6, 57-66

Müller M., 1991. Frequency representation in the rat cochlea. Hear. Res. 51, 247-254.

Neely S.T., Kim D.O., 1983. An active cochlear model showing sharp tuning and high sensitivity. Hear. Res. 9, 123-130.

Passchier-Vermeer W., Smoorenburg G.F., 1994. Noise and health, Health Council of the Netherlands, Committee on Noise and Health, The Hague, ISBN 90-5549-048-02, 1-101.

Patuzzi R.B., Yates G.K., Johnstone B.M., 1989. Outer hair cell receptor current and sensorineural hearing loss. Hear. Res. 42, 47-72.

Patuzzi R. M., 1996. Cochlear micromechanics and macromechanics. In: Dallos P., Popper A.N., Fay R.R. (Eds.), The Cochlea. Springer Verlag, New York, pp 186-257.

Pickles J.O., 1988. An introduction to the physiology of hearing. $2^{\text {nd }}$ edition, Academic Press, London.

Probst R., Lonsbury-Martin B.L., Martin G.K., 1991. A review of otoacoustic emissions. J. Acoust. Soc. Am. 89, 2027-2067.

Pryor G.T., Dickinson J., Howd R.A., Rebert C.S., 1983. Transient cognitive deficits and high-frequency hearing loss in weanling rats exposed to toluene. Neurobehav. Toxicol. Teratol. 5, 53-57.

Pryor G.T., Dickinson J., Feeney E., Rebert C.S., 1984. Hearing loss in rats first exposed to toluene as weanlings or as young adults. Neurobehav. Toxicol. Teratol. 6,111-119.

Pryor G.T., Rebert C.S., Howd R.A., 1987. Hearing loss in rats caused by inhalation of mixed xylenes and styrene. J. Appl. Toxicol. 7, 55-61. 
Pryor G.T., Rebert C.S., 1993. Neurotoxicology of inhaled substances. Final Report NIDA contract 271-90-7202, SRI International, Menlo Park, CA.

Pryor G.T., 1994. Assessment of auditory dysfunction. In: L.W. Chang (Ed.) Principles of neurotoxicology. Marcel Dekker Inc, New York, NY, pp 345-371.

Pryor G.T., 1995. Solvent-induced neurotoxicity: Effects and mechanisms. In: L.W. Chang (Ed.) Handbook of neurotoxicology. Marcel Dekker Inc, New York, pp 377-400.

Puel J.-L., Ruel J., Gervai d'Alpin C., Pujol R., 1998. Excitotoxicity and repair of cochlear synapses after noise-trauma induced hearing loss. Neuroreport 9, 2109-2114.

Rebert C.S., Sorensen S.S., Howd R.A., Pryor G.T., 1983. Toluene-induced hearing loss in rats evidenced by the brainstem auditory-evoked response. Neurobehav. Toxicol. Teratol. 5, 59-62.

Rebert C.S., Day V.L. Matteucci M.J., Pryor G.T., 1991. Sensory-evoked potentials in rats chronically exposed to trichloroethylene: Predominant auditory dysfunction. Neurotoxicol. Teratol. 13, 83-90.

Rebert C.S., Boyes W.K., Pryor G.T., Svensgaard D.J., Kassay K.M., Gordon G.R., Shinsky N., 1993. Combined effects of solvents on the rat's auditory system: Styrene and trichloroethylene. Int. J. Psychophysiol. 14, 49-59.

Rebert C.S., Hall T.A., 1994. The neuroepidemiology of styrene: A critical review of representative literature. Crit. Rev. Toxicol. 24 (S1), S57-S106.

Robertson D., Johnstone B.M., McGill T.J., 1980. Effects of loud tones on the inner ear: A combined electrophysiological and ultrastructural study. Hear. Res. 2, 39-53.

Robertson D., 1983. Functional significance of dendritic swelling after loud sounds in the guinea pig cochlea. Hear. Res. 9, 263-278.

Robles L., Ruggero M.A., Rich N.C., 1991. Two-tone distortion in the basilar membrane of the cochlea. Nature 349, 413-414.

Rybak L.P., 1992. Hearing: The effects of chemicals. Otolaryngol. Head Neck Surg. 106, 677-686.

Salvi R.J., Henderson D., Eddins A.C., 1995. Effects of noise exposure on the auditory system. In: Chang L.W. (Eds.), Handbook of neurotoxicology. Marcel Dekker Inc, NY, pp 907-961.

Sass-Kortsak A.M., Corey P.N., Robertson J.M., 1995. An investigation of the association between exposure to styrene and hearing loss. Ann. Epidemiol. 5, 15-24.

Saunders J.C., Dear S.P., Schneider M.E., 1985. The anatomical consequences of acoustics injury: A review and tutorial. J. Acoust. Soc. Am. 78, 833-860.

Schoonhoven R., Prijs V.F., Grote, J.J., 1996. Response thresholds in electrocochleography and their relation to the pure tone audiogram. Ear \& Hearing 17, 266-275.

Schoonhoven R., Lamoré P.J.J., de Laat J.A.P.M., Grote J.J., 2000. Long term audiometric follow-up of click-evoked ABR in hearing impaired infants. Audiology, in press.

Sellick P.M., Patuzzi R., Johnstone B.M., 1982. Modulation of responses of spiral ganglion cells in the guinea pig cochlea by low frequency sound. Hear. Res. 7, 199-221.

Slepecky N., 1986. Overview of mechanical damage to the inner ear: noise as a tool to probe cochlear function. Hear. Res. 22, 307-321.

Spencer P.S., Schaumburg H.H., 1985. Organic solvent neurotoxicity. Scand. J. Work Environ. Health $11,53-60$. 
Spoendlin H., Brun J.-P., 1974. The block-surface technique for evaluation of cochlear pathology. Arch. Oto-Rhino-Laryngol. 208, 137-145.

Spoendlin H., 1988. Neural anatomy of the inner ear. In: Jahn A.F., Santos-Sacchi J., (Eds.) Physiology of the ear. Raven Press, New York, pp 201-209.

Stengs C.H.M., Klis S.F.L., Huizing E.H., Smoorenburg G.F., 1997. Cisplatin-induced ototoxicity; Electrophysiological evidence of spontaneous recovery in the albino guinea pig. Hear. Res. 111, 103-113.

Subramaniam M., Henderson D., Spongr V., 1994. The relationship among distortion-product otoacoustic emissions, evoked potential thresholds, and outer hair cell loss following interrupted noise exposures. Ear \& Hearing 15, 299-309.

Subramaniam M., Henselman L.W., Sprongr V., Henderson D., Powers N.L., 1995. Effect of highfrequency interrupted noise exposures on evoked-potential thresholds, distrotion-product otoacoustic emissions, and outer hair cell loss. Ear \& Hearing 16, 372-381.

Sullivan M.J., Conoly R.B., 1988. Dose-response hearing loss for white noise in the Sprague-Dawley rat. Fund. Appl. Toxicol. 10, 109-113.

Sullivan M.J., Rarey K.E., Conolly R.B., 1989. Ototoxicity of toluene in rats. Neurotoxicol. Teratol. 10, 525-530.

Syka J., Melchiar I., Ulehlová L., 1981. Longitudinal distribution of cochlear potentials and the $\mathrm{K}^{+}$ concentration in the endolymph after acoustic trauma. Hear. Res. 4, 287-298.

Szulc-Kuberska J., Tronczynska J., Latkowski B., 1976. Oto-neurological investigations of chronic trichlorethylene poisoning. Minerva Otorinolaringol. 26, 108-112.

Tarnowski B.I., Schmiedt R.A., Hellstrom L.I., Lee F.S., Adams J.C., 1991. Age-related changes in cochleas of Mongolian gerbils. Hear. Res. 54, 123-134.

van der Drift J.F.C., Brocaar M.P. van Zanten G.A., 1987. The relation between the pure tone audiogram and the click auditory brainstem response threshold in cochlear hearing loss. Audiology 26, 1-10.

Vertes D., Axelsson A., Miller J., Lidén G., 1981. Cochlear vascular and electrophysiological effects in the guinea pig to $4 \mathrm{kHz}$ pure tones of different durations and intensities. Acta Otolaryngol. $92,15-24$.

Von Bekesy G., 1960. Experiments in hearing. McGraw-Hill, New York.

Ward W.D., Santi P.A., Duvall A.J., Turner C.W., 1981. Total energy and critical intensity concepts in noise damage. Ann. Otol. 90, 584-590.

Yamamura K., Ikeda T., Sadamoto T., Maehara N., Harabuchi I., Takashima H., Kiyosawa H., 1983. Effects of trichloroethylene exposure on hearing. Eur. J. Appl. Physiol. 52, 47-50.

Yano B.L., Dittenber D.A., Albee R.R., Mattsson J.L., 1992. Abnormal auditory brainstem and cochlear pathology in rats induced by an exaggerated styrene exposure regimen. Toxicol. Pathol. 20, 16.

Yoshida N., Liberman M.C., 1999. Sterociliary anomaly in the guinea pig: effects of hair bundle rotation on cochlear sensitivity. Hear. Res. 131, 29-38. 



\section{SAMENVATTING \& ConClusies}


Gehoorverlies als gevolg van blootstelling aan lawaai is een notoir probleem in geindustrialiseerde landen. Een veel minder bekend en begrepen probleem is gehoorverlies als gevolg van blootstelling aan organische oplosmiddelen. Er komt steeds meer bewijs dat gecombineerde blootstelling aan lawaai en organische oplosmiddelen een groter gehoorverlies kan veroorzaken dan kan worden voorspeld op grond van blootstelling aan lawaai of oplosmiddelen alleen.

In dit proefschrift is een diermodel ontwikkeld waarmee de schadelijkheid van het oplosmiddel ethylbenzeen bepaald kan worden. Tegelijkertijd wordt nagegaan hoe deze stof inwerkt op structuren van het binnenoor. Vervolgens zijn de mogelijke synergistische interacties tussen lawaai en ethylbenzeen op het gehoor onderzocht.

Hoofdstuk 1, de inleiding, begint met een samenvatting over de werking van het perifere auditieve systeem. Vervolgens worden de meetmethoden die in dit proefschrift gebruikt zijn besproken. Er is gebruik gemaakt van een gedragsbepaalde meetmethode (reflex modification audiometry; RMA), van electrocochleografie (compound action potentials; CAP), het meten van akoestische vervormingsproducten (distortion product otoacoustic emissions; DPOAE) en van histologische technieken. Verder wordt in de inleiding een overzicht gegeven van de literatuur over de effecten van lawaai, organische oplosmiddelen en de combinatie van lawaai en oplosmiddelen op het auditief systeem.

Hoofdstuk 2 beschrijft de effecten van ethylbenzeen op het auditieve systeem. Ratten werden 5 dagen lang, 8 uur per dag blootgesteld aan 800 ppm ethylbenzeen. Ethylbenzeen veroorzaakte een gehoorverlies van ongeveer $20 \mathrm{~dB}$ in het frequentiebereik dat werd getest $(4-24 \mathrm{kHz})$. Dit werd vastgesteld met RMA en CAP metingen. Het aantal aanwezige buitenste haarcellen werd geteld in mid-modiolaire coupes van de cochlea. Ongeveer 60\% was verloren gegaan in het gebied van de cochlea corresponderend met $11-21 \mathrm{kHz}$; het middenfrequente gebied in de rat. Het haarcelverlies en de CAP gegevens wijzen erop dat ethylbenzeen specifiek het perifere auditieve systeem aantast. De buitenste haarcellen blijken het voornaamste doelwit te zijn. Effecten op binnenste haarcellen werden niet gezien.

In vergelijking met andere organische oplosmiddelen bleek ethylbenzeen zeer potent te zijn. Tolueen, styreen en trichloorethyleen moeten langer en/of in een hogere concentratie worden gedoseerd om effecten teweeg te brengen die 
vergelijkbaar zijn met de effecten die werden veroorzaakt door ethylbenzeen in deze studie.

Het belangrijkste doel van de dosis-effect studie beschreven in Hoofdstuk 3 was de minimale concentratie ethylbenzeen te bepalen die ototoxisch is in de rat. Ratten werden blootgesteld aan 300, 400 of 550 ppm ethylbenzeen (8 uur/dag, 5 dagen). Het gehoorverlies werd bepaald met behulp van DPOAE en CAP metingen. Het buitenste haarcelverlies werd bepaald in mid-modiolaire coupes. De minimale concentratie ethylbenzeen die nodig was om gehoorverlies te veroorzaken was 400 ppm. Het gehoorverlies werd gevonden in het middenfrequente bereik. Met oplopende concentratie ethylbenzeen (400 tot 800 ppm, zie ook Hoofdstuk 2) nam de ernst van het gehoorverlies toe, het aantal verdwenen haarcellen werd groter en het frequentiebereik waarover de schade zich uitstrekte verbreedde. Met deze studie werd een basis gelegd voor de studies waarin lawaai en ethylbenzeen gelijktijdig worden gedoseerd. Ook werd de uitspraak dat ethylbenzeen zeer ototoxisch is verder gestaafd.

In Hoofdstuk 4 wordt een dosis-effect studie besproken waarin de effecten van breedbandige ruis op het gehoor van de rat worden onderzocht. De ruis werd aangeboden op drie geluidniveaus. Het doel van deze studie was een basis te leggen voor de combinatiestudie en gegevens toe te voegen aan de vrij kleine dataset over de gevolgen van langdurige blootstelling aan lawaai op het gehoor van ratten.

Ratten werden blootgesteld aan 90, 100 of $110 \mathrm{~dB}$ SPL breedbandige ruis. Het protocol was identiek aan het protocol dat gebruikt werd in de dosis-effect studie van ethylbenzeen (Hoofdstuk 3). Het gehoorverlies werd bepaald door middel van DPOAE en CAP metingen. Bovendien werd het aantal aanwezige buitenste haarcellen geteld in mid-modiolaire coupes.

Alleen de twee hoogste geluidniveaus veroorzaakten gehoorverlies. De CAP meting na ruis van $100 \mathrm{~dB}$ SPL liet enkel bij $12 \mathrm{kHz}$ een verlies zien. Een groter gehoorverlies werd gevonden na $110 \mathrm{~dB}$ SPL en ook het frequentiebereik van het verlies breidde zich uit $(4-24 \mathrm{kHz})$. Dit functionele gehoorverlies werd niet vergezeld van buitenste haarcelverlies. Deze discrepantie kan veroorzaakt zijn doordat lawaai subcellulair schade heeft veroorzaakt, die lichtmicroscopisch niet waarneembaar is. Lawaai zou de stereocilia van binnenste haarcellen en de binnenste rij buitenste haarcellen kunnen beschadigen en niet het cellichaam. 
De resultaten van deze studie werden vergeleken met andere studies waarin ratten werden blootgesteld aan lawaai voor periodes langer dan één dag. Als algemene maat voor geluidniveau werd het totale expositieniveau berekend op grond van de "equal-energy" theorie. De vergelijking vertoont een consistente relatie: hoe hoger het in de totale energie uitgedrukte expositieniveau, des te groter het gehoorverlies. Deze relatie is consistent ondanks het gegeven dat er verschillende typen lawaai, verschillende protocollen en verschillende testmethoden werden gebruikt in de betrokken studies. Het resultaat toont aan dat de "equal-energy" theorie gebruikt kan worden om gehoorverliezen als gevolg van blootstelling aan lawaai te voorspellen in ratten.

In Hoofdstuk 5 worden de effecten van gecombineerde blootstelling aan lawaai en ethylbenzeen op het gehoor van de rat beschreven. De ethylbenzeen concentraties en de geluidniveaus die werden gebruikt in deze studie zijn gebaseerd op de dosis-effect studies in de Hoofdstukken 3 en 4 . De gekozen concentraties en niveaus waren relatief laag, omdat we geïnteresseerd waren in kleine effecten van lawaai en ethylbenzeen afzonderlijk. Iedere agens op zich wordt onschadelijk geacht, maar in combinatie zouden er wel schadelijke effecten kunnen optreden. De effecten van 3 concentraties ethylbenzeen (0,300 of $400 \mathrm{ppm}$ ), drie lawaainiveaus (95 of 105 dB SPL of achtergrond ruis) en alle mogelijke combinaties werden onderzocht. De effecten op het perifere auditieve systeem werden onderzocht met behulp van DPOAE en CAP metingen en haarceltellingen in mid-modiolaire coupes. Aanvullend op de mid-modiolaire haarceltellingen zijn er in deze studie ook oppervlaktepreparaten gemaakt waarin het aantal haarcellen over de gehele basilair membraan bepaald kan worden. Met deze techniek kan onderscheid gemaakt worden tussen verliezen in de verschillende rijen haarcellen.

Ethylbenzeen afzonderlijk, 300 of $400 \mathrm{ppm}$, veroorzaakte geen functioneel gehoorverlies. Er is een kleine discrepantie tussen deze gegevens en de gegevens van het experiment in Hoofdstuk 3. Daar werd wel een gering gehoorverlies gevonden na $400 \mathrm{ppm}$ ethylbenzeen. Overeenkomend met Hoofdstuk 3 daarentegen is het buitenste haarcelverlies. Nieuw is dat we nu kunnen concluderen dat dit verlies voornamelijk te vinden was in de derde rij buitenste haarcellen. De buitenste haarcellen in eerste en tweede rij waren minder getroffen. De DPOAE en CAP metingen vertoonden gehoorverlies na $105 \mathrm{~dB}$ SPL lawaai, maar slechts een zeer klein 
percentage buitenste haarcellen was verdwenen in de eerste rij, overeenkomstig de resultaten in Hoofdstuk 4. Gecombineerde blootstelling aan ethylbenzeen (300 en 400 ppm) en $95 \mathrm{~dB}$ SPL lawaai veroorzaakte geen gehoorverlies. Als het lawaainiveau werd verhoogd naar $105 \mathrm{~dB}$ SPL in combinatie met ethylbenzeen werd er een matig gehoorverlies gevonden. Dit verlies was echter niet groter dan het gehoorverlies veroorzaakt door $105 \mathrm{~dB}$ SPL lawaai alleen.

Dus, op basis van de functionele metingen werden er geen aanwijzingen gevonden voor een synergistische interactie tussen lawaai en ethylbenzeen. In de haarceltellingen daarentegen was er een duidelijk synergistisch effect. Dit effect werd gevonden rond de meest beschadigde gebieden van de basilair membraan en deed zich voor in alle rijen buitenste haarcellen.

De eerste vier studies die zijn beschreven in dit proefschrift, zijn gebaseerd op de rat als diermodel. De ototoxiciteit van oplosmiddelen blijkt echter afhankelijk te zijn van de diersoort. Cavia's vertonen in het algemeen minder of geen effect van oplosmiddelen op het gehoor. Geïnspireerd door de hoge ototoxiciteit van ethylbenzeen in ratten, werd besloten de ototxiciteit van ethylbenzeen in cavia's te bepalen. De resultaten van dit experiment zijn beschreven in Hoofdstuk 6. Allereerst is er een oriënterend experiment uitgevoerd, waarin cavia's 8 uur per dag, 5 dagen per week werden blootgesteld aan ethylbenzeen. In de eerste week was de concentratie ethylbenzeen $800 \mathrm{ppm}$, in de tweede week werd de concentratie verhoogd naar 1600 ppm en in de derde en laatste week werd de concentratie nog verder verhoogd naar 2000 ppm. De cavia's werden na elke blootstellingsweek getest op gehoorverlies door middel van de gedragsmethode (RMA), maar er werden geen gehoorverliezen gevonden. Ook werden er na afloop van de drie weken blootstelling geen gehoorverliezen gevonden met DPOAE en CAP metingen. Bovendien werd er geen haarcelverlies gedetecteerd.

In een tweede experiment werden cavia's blootgesteld aan 2500 ppm ethylbenzeen ( 6 uur per dag, 5 dagen). Deze voor de cavia bijna letale concentratie veroorzaakte geen schade aan het perifere auditieve systeem. Dit werd aangetoond met zowel de CAP metingen als met de haarceltellingen.

Uit de twee voorgaande experimenten kunnen we concluderen dat cavia's veel minder gevoelig zijn voor hoge concentraties ethylbenzeen dan ratten. In een poging om deze verschillen te verklaren, is een kleine studie uitgevoerd waarin zowel ratten 
als cavia's werden blootgesteld aan 500 ppm ethylbenzeen (8 uur per dag, 3 dagen). Aan het eind van dag 1 en dag 3 werden bloedmonsters verzameld. De concentratie ethylbenzeen in bloed op het eind van dag 1 was in de rat 8.3 keer zo groot als in de cavia. Hoewel de absolute concentratie in zowel de rat als de cavia was gedaald op dag 3, was de concentratie in het bloed van de rat nog steeds 4.3 keer zo groot als de concentratie ethylbenzeen in de cavia. De verschillen in gevoeligheid voor ethylbenzeen zouden verklaard kunnen worden door verschillen in opname, distributie, metabolisme en/of uitscheiding van ethylbenzeen.

Een belangrijke vraag is de betekenis van dierstudies voor het voorspellen van ototoxiciteit van oplosmiddelen in de mens. Het feit dat mensen gevoelig zijn voor oplosmiddelen en cavia's niet, pleit ervoor de rat als model te gebruiken.

De Hoofdstukken 7 en 8 handelen over bepaalde details van gegevens in de eerder beschreven hoofdstukken. In Hoofdstuk 7 wordt de relatie tussen haarcelverlies en functioneel gehoorverlies verder onderzocht in een subset van de data beschreven in Hoofdstuk 5. Het buitenste haarcelverlies per rij na blootstelling aan $300 \mathrm{ppm}$ ethylbenzeen, $400 \mathrm{ppm}$ ethylbenzeen en $105 \mathrm{~dB}$ SPL lawaai werd vergeleken met het CAP gehoorverlies in dezelfde oren. Er kan een groot haarcelverlies optreden in de derde rij buitenste haarcellen zonder dat er functioneel gehoorverlies optreedt. Aan de andere kant kan er een groot functioneel gehoorverlies zijn met een klein verlies van buitenste haarcellen in de eerste rij. Klaarblijkelijk is de eerste rij buitenste haarcellen belangrijker dan de derde rij voor het goed functioneren van het auditief systeem.

Hoofdstuk 8 is het resultaat van een samenwerking met de universiteiten van Leiden en Rotterdam. Dit hoofdstuk beschrijft de relatie tussen twee methoden om gehoorverlies te bepalen: gedragsmetingen, zoals het klinisch bepalen van een audiogram, en objectieve testmethoden, zoals die gebaseerd op auditief opgewekte potentialen. Het auditieve systeem integreert de binnenkomende geluidsenergie over korte tijdsintervallen. Door deze eigenschap hangt de gehoordrempel af van de duur van de aangeboden geluidsstimulus. De drempel wordt $10 \mathrm{~dB}$ per decade beter bij een stimulusduur oplopend tot $200-300 \mathrm{~ms}$. Bij langere stimuli is de drempel constant. Als gevolg van pathologische processen kan een degradatie optreden van de temporele integratie. Dit wil zeggen dat het verschil tussen gehoordrempels gevonden met korte versus langere stimuli kleiner kan zijn in beschadigde oren dan in gezonde oren. 
Auditief opgewekte potentialen worden gemeten met kortdurende toonstimuli of klikken. Gehoordrempels bepaald met gedragmetingen zijn daarentegen in het algemeen gebaseerd op langdurige stimuli. Dit verschil kan leiden tot een systematische fout wanneer men de gehoordrempels wil voorspellen uit metingen aan auditief opgewekte potentialen. Deze fout neemt toe bij een toenemend gehoorverlies. In dit hoofdstuk is dit probleem onderzocht met behulp van 4 datasets, die bestonden uit gedragsmetingen en elektrofysiologische metingen aan dezelfde oren. Drie van de vier sets werden verzameld in de kliniek in patiënten met veel en weinig gehoorverlies, de vierde set was afkomstig van de rat en is eerder besproken in Hoofdstuk 2. De conclusie is dat in alle datasets de gehoordrempel hoger is als gevolg van verschillende pathologische processen dan de drempel die de auditief opgewekte potentialen zou voorspellen. Deze conclusie gold ook voor de dataset verzameld in ratten, ondanks het feit dat het in deze set reflex data betrof waarbij de rol van temporele integratie niet bekend is.

\section{Algemene conclusies}

I. Ethylbenzeen is in vergelijking met andere organische oplosmiddelen een zeer potent ototoxisch agens in de rat.

II. Cavia's zijn veel minder gevoelig voor de ototoxische effecten van ethylbenzeen dan ratten.

III. Lage concentraties ethylbenzeen veroorzaken een buitenste haarcelverlies in de derde rij, zonder functioneel gehoorverlies.

IV. Lage lawaai niveaus veroorzaken functionele gehoorverliezen met lichtmicroscopisch onzichtbare schade aan de haarcellen.

V. Een vergelijking tussen gehoordrempels gebaseerd op gedrag en gehoordrempels gebaseerd op auditief opgewekte potentialen laat zien dat de laatsten de gehoorverliezen systematisch onderschatten.

VI. Onder speciale omstandigheden kan er een synergistische interactie optreden tussen lawaai en ethylbenzeen, in het bijzonder op het niveau van de haarcellen. 


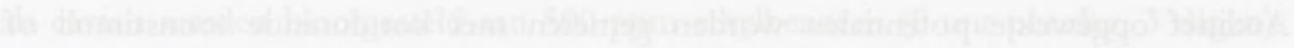

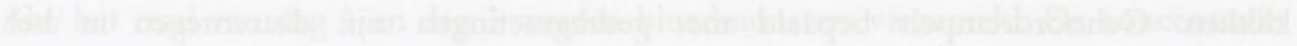

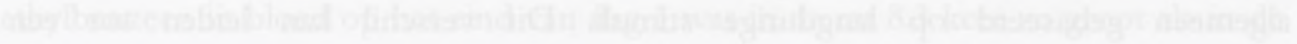

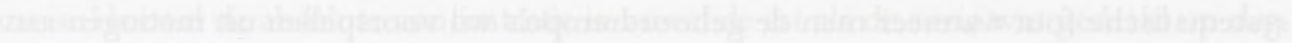

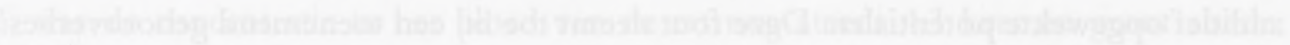

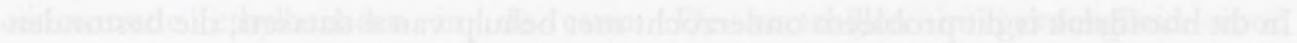

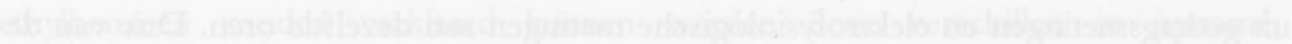

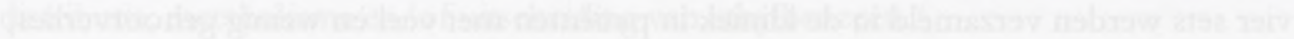

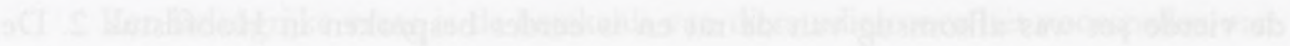

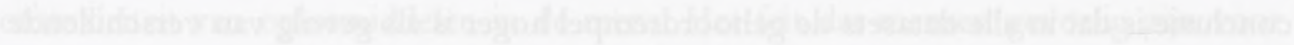

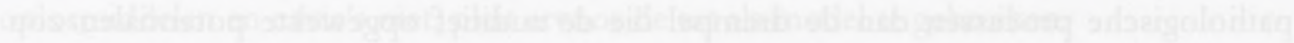

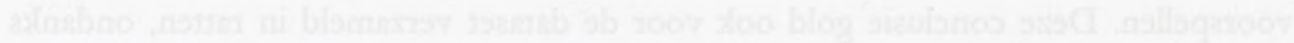

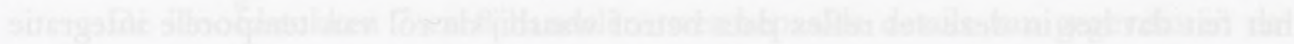




\section{BEDANKT}

Het maken van het proefschrift is niet niks. Toch ik heb nooit het idee gehad dat ik er alleen voor stond, dankzij de steun en hulp die ik van veel mensen, zowel collega's als vrienden en familie, gekregen heb. Iedereen die op wat voor manier dan ook heeft bijgedragen aan dit boekje: BEDANKT!

Hieronder wil ik een aantal mensen in het bijzonder bedanken. Allereerst de AZU-ers: Prof. Smoorenburg, promotor, bedankt voor de ondersteuning waar nodig en het kritisch lezen van de artikelen.

Sjaak, de altijd kritische co-promotor. Dat kritische leidde vaak tot heel wat discussie, maar we kwamen er altijd wel weer uit, meestal met de grootste schik.

Alle AZU-collega's door jaren heen, het was altijd erg gezellig en ik ging dan ook altijd met veel plezier werken. Naast wetenschap was er ook altijd tijd voor andere zaken.

Anne Baretta, door jouw enthousiasme en ideeën was erg leuk om met jouw samen te werken.

De collega's van het histo-lab, bedankt voor de onontbeerlijke hulp op het gebied van de histologie, zonder jullie zou er geen haarcel geteld zijn.

ID-ers van het AZU, bedankt voor alle technische en elektronische ondersteuning.

\section{De TNO-ers:}

Hans, co-promoter van TNO, ondanks alle drukte op TNO kon je altijd wel ergens tijd voor mij vrij maken, bedankt.

Beverly, bedankt voor de vrijheid die je mij gegeven hebt bij het uitvoeren van het onderzoek.

AIO's van de aio-kamer in brede zin, soms was het iets te gezellig en kwam er niet veel meer van werken. Maar wel leuk!

Dierverzorgers van TNO, bedankt voor de goede zorgen.

$\mathrm{Pa}$ en ma, ondanks mijn "van zessen klaar" strategie hebben jullie altijd het grootste vertrouwen in mij en staan jullie altijd voor mij klaar, dat is erg fijn. 


\section{CURRICULUM VITAE}

Naam: Natalie Cappaert

Geboortedatum: $\quad 5$ oktober 1973

Middelbare school: $\quad$ 1985-1991

VWO Jansenius Scholengemeenschap Hulst

Studie biologie: $\quad$ 1991-1996, K.U. Nijmegen

Stages:

-Vakgroep Medische Fysica \& Biofysica, K.U. Nijmegen.

Karakteriseren van oog-hoofdbewegingen naar auditieve en visuele doelen.

-Rudolf Magnus Instituut, Universiteit Utrecht.

Effecten van stress op leren en geheugen en op "long-termpotentiation" in de hippocampus.

Promotieonderzoek: 1996-2000

Vakgroep Experimentele Audiologie, Universitair Medisch Centrum Utrecht en TNO Voeding, Zeist.

\section{LIST OF PUBLICATIONS}

\section{Papers}

Cappaert N.L.M., Klis S.F.L., Muijser H., De Groot J.C.M.J., Kulig B.M., Smoorenburg G.F., 1999. The ototoxic effects of ethyl benzene in rats. Hear. Res.137, 91-102.

Cappaert N.L.M., Klis S.F.L., Muijser H., Kulig B.M., Smoorenburg G.F., 2000. Noiseinduced hearing loss in rats. Noise \& Health, in press. 
Schoonhoven R., Cappaert N.L.M., van Zanten G.A., 2001. Pure tone versus auditory evoked potential thresholds in cochlear hearing loss: Manifestations of degrading temporal integration. In: Houtsma A.J.M., Kohlrausch A., Prijs V.F., Schoonhoven R. (Eds.), Physiological and Psychophysical Bases of Auditory Function, Proceedings of the $12^{\text {th }}$ International Symposium on Hearing. Shaker Publishing BV, Maastricht, in press.

Cappaert N.L.M., Klis S.F.L., Baretta A.B., Muijser H., Smoorenburg G.F. Ethyl benzeneinduced ototoxicity in rats: A dose-dependent mid-frequency hearing loss. Accepted by JARO.

Cappaert N.L.M., Klis S.F.L., Muijser H., Kulig B.M., Smoorenburg G.F. Effects of simultaneous exposure to ethyl benzene and broad-band noise on hearing in rats. Submitted for publication in Hearing Research.

Cappaert N.L.M., Klis S.F.L., Muijser H., Kulig B.M., Ravensberg L.C., Smoorenburg G.F. Rats are highly susceptible to the organic solvents ethyl benzene whereas guinea pigs are not. Submitted for publication in Neurotoxicology and Teratology.

Smoorenburg G.F., Cappaert N.L.M., Klis S.F.L. The effects of simultaneous exposure to ethyl benzene and noise on hearing. Will be published in the Proceedings of the International Symposium on Noise Induced Hearing loss 2000, Cambridge, UK.

\section{Abstracts}

Cappaert N.L.M., Klis S.F.L., Muijser H., Kulig B.M., Smoorenburg G.F., 1997. Het effect van ethylbenzeen op het gehoor van de rat. Ned. Tijdschrift KNO-Heelkunde Jaargang 3, 167-168.

Cappaert N.L.M., Klis S.F.L., Muijser H., De Groot J.C.M.J., Kulig B.M., Smoorenburg G.F., 1997. The effects of ethyl benzene exposure on hearing in rats. Neurotoxicol. Ass. 883.

Cappaert N.L.M., Klis S.F.L., Muijser H., Kulig B.M., Smoorenburg G.F., 1998. The effects of ethyl benzene exposure on hearing in rats. Clinical Otolaryngol. 23, 379. 


\section{8}

Cappaert N.L.M., Klis S.F.L., Muijser H., Kulig B.M., Smoorenburg G.F., 1999. Ototoxic effects of the organic solvent ethyl benzene in of rats and guinea pigs. $35^{\text {th }}$ Workshop on Inner Ear Biology, Innsbruck, Austria.

Cappaert N.L.M., Klis S.F.L., Muijser H., Kulig B.M., Smoorenburg G.F., 1999. Different susceptibility of rats and guinea pigs to ototoxic effects of ethyl benzene. Proceedings 7 th International Audiologic Symposium and European Conference Pan'99. Zakopane, Poland.

Cappaert N.L.M., Klis S.F.L., Muijser H., Kulig B.M., Smoorenburg G.F., 2000. Rats are highly susceptible to the ototoxic effects of the organic solvent ethyl benzene and guinea pigs are not. Abstract book $23^{\text {th }}$ Midwinter Meeting of the Association for Research in Otorhinolaryngology, St. Petersburg Beach, Florida.

Cappaert N.L.M., Klis S.F.L., Muijser H., Kulig B.M., Smoorenburg G.F., 2000. The effects of simultaneous exposure to ethyl benzene and noise on hearing. Abstract book NOPHER 2000, An International Symposium on Noise-Induced Hearing Loss, Cambridge, UK. 

